\title{
Validation and Testing of the VAM2D Computer Code
}

Manuscript Completed: September 1991

Date Published: October 1991

Prepared by

J. B. Kool, Y. S. Wu

T. J. Nicholson, NRC Project Manager

HydroGeoLogic, Inc.

1165 Herndon Parkway

Suite 900

Herndon, VA 22070

Prepared for

Division of Regulatory Applications

Office of Nuclear Regulatory Research

U.S. Nuclear Regulatory Commission

Washington, DC 20555

NRC FIN L1273 


\begin{abstract}
This document describes two modeling studies conducted by HydroGeoLogic, Inc. for the U.S. NRC under contract no. NRC-04-89-090, entitled, "Validation and Testing of the VAM2D Computer Code." VAM2D is a two-dimensional, variably saturated flow and transfort code, with applications for performance assessment of nuclear waste disposal. The computer code itself is documented in a separate NUREG document (NUREG/CR-5352, 1989). The studies prsented in this report involve application of the VAM2D code to two diverse subsurface modeling problems. The first one involves modeling of infiltration and redistribution of water and solutes in an initially dry, heterogeneous field soil. This application involves detailed modeling over a relatively short, 9-month time period. The second problem pertains to the application of VAM2D to the modeling of a waste disposal facility in a fractured clay, over much larger space and time scales and with particular emphasis on the applicability and reliability of using the equivalent porous medium approach for simulating flow and transport in fractured geologic media. Reflecting the separate and distinct nature of the two problems studied, this report is organized in two separate parts.
\end{abstract}




\section{TABLE OF CONTENTS}

Section

Page

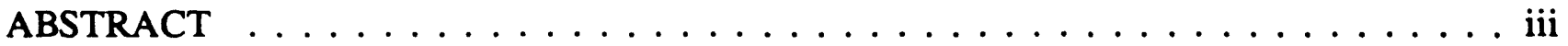

ACKNOWLEDGEMENT $\ldots \ldots \ldots \ldots \ldots \ldots \ldots \ldots \ldots \ldots$

PART 1 APPLICATION OF THE VAM2D CODE TO THE SECOND LAS CRUCES TRENCH EXPERIMENT

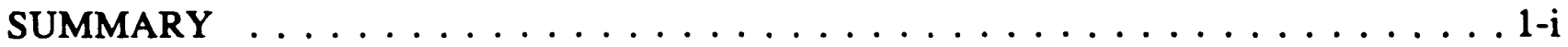

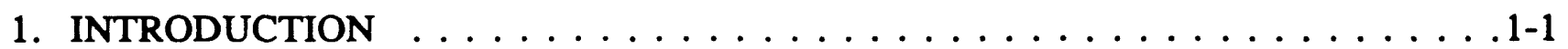

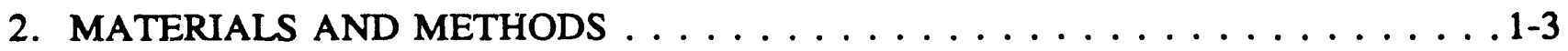

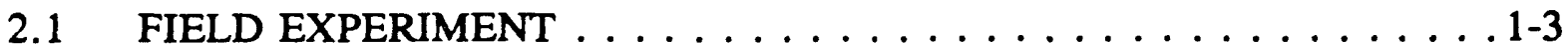

2.2 FLOW AND TRANSPORT MODEL ................. . . . . . . . . .

2.2 .1 Governing Equations . . . . . . . . . . . . . . . . 1-6

2.2.2 Numerical Procedures for Efficient Flow and Transport

Simulation ......................... . . . . . . .

2.2.3 Model Domain and Boundary Conditions ........... 1-13

2.2.4.1 Scenario I . . . . . . . . . . . . . . . . 1-17

2.2.4.2 Scenario IIa . . . . . . . . . . . . . . . . 1-19

2.2.4.3 Scenario IIb . . . . . . . . . . . . . . . . 1-19

2.2.4.4 Scenario IIc . . . . . . . . . . . . . . . . 1-21

2.2.4.5 Scenario III . . . . . . . . . . . . . . . . 1-21

2.2.4.6 Solute Transport Parameters ... . . . . . . . . . 1-21

2.3 MODEL EVALUATION CRITERIA . . . . . . . . . . . . . . 1-24

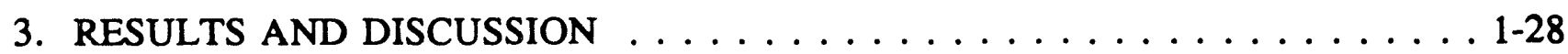

3.2 SOLUTE TRANSPORT MODELING $\ldots \ldots \ldots \ldots \ldots \ldots \ldots$

4. CONCLUSIONS $\ldots \ldots \ldots \ldots \ldots \ldots \ldots \ldots \ldots \ldots \ldots$

PART 2 APPLICATION OF THE VAM2D COMPUTER CODE FOR MODELING OF FLOW AND TRANSPORT IN FRACTURED MEDIA USING AN EQUTVALENT POROUS MEDIUM APPROACH

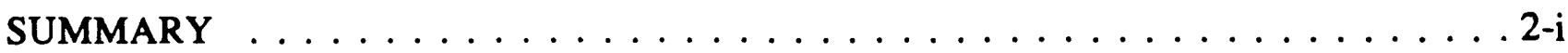

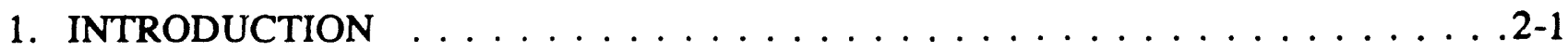


2. METHODOLOGY . . . . . . . . . . . . . . . . . . . 2-4

2.1 DESCRIPTION OF WASTE DISPOSAL SITE $\ldots \ldots \ldots \ldots \ldots \ldots .2-4$

2.2 FRACTRAN DISCRETE FRACTURE FLOW AND TRANSPORT

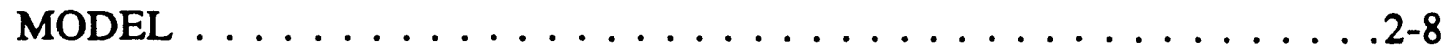

2.2.1 Model Formulation . . . . . . . . . . . . . . . 2-8

2.2.2 Solution Technique . . . . . . . . . . . . . 2-10

2.3 EPM APPROXIMATIONS FOR FLOW AND TRANSPORT

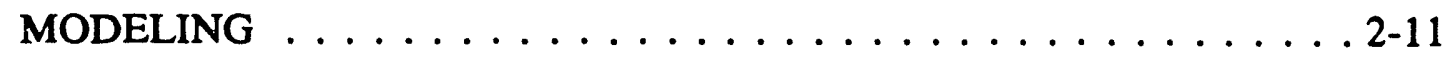

2.4 FINITE ELEMENT DISCRETIZATION FOR EPM MODELING $\ldots \ldots$ 2-18

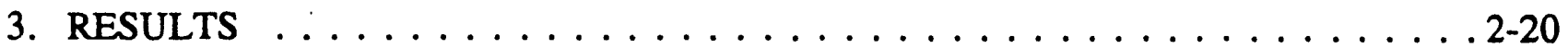

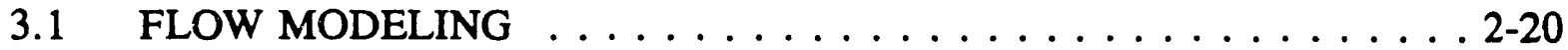

3.2 TRANSPORT MODELING $\ldots \ldots \ldots \ldots \ldots \ldots \ldots \ldots \ldots \ldots \ldots$

3.2.1 Transport Scenarios . . . . . . . . . . . . 2-20

3.2.2 Conservative Contaminant Transport . . . . . . . . 2-25

3.2.3 Reactive Contaminant Transport . . . . . . . . . . 2-33

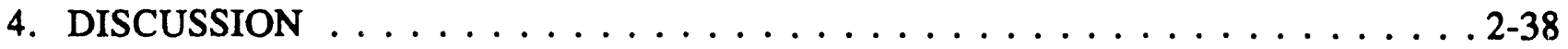

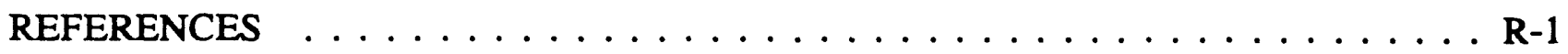

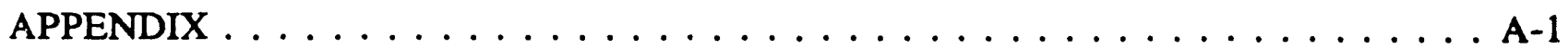




\section{LIST OF FIGURES}

Figure

Page

PART 1 APPLICATION OF THE VAM2D CODE TO THE SECOND LAS CRUCES TRENCH EXPERIMENT

1. Deposits and soil horizons present in upper $6 \mathrm{~m}$ of the soil at the Jornada experimental site. Circles indicate the depths from which samples were taken (from Wierenga et al., 1989). . . . . . . . . . . . . . . . . . 1-4

2. Plan view of the Las Cruces trench site (from Wierenga et al., 1989). . . . . . . 1-5

3. Cross-sectional view showing location of tensiometcrs and solute samplers underneath the irrigation plot (from Wierenga et al., 1990). . . . . . . . . 1-7

4. Schematic view of the modeled two-dimensional cross-section and assignment of boundary conditions. ........................ . . . . . . .

5. Finite element discretization used in VAM2D flow and transport simulations. . 1-16

6. Initial water content distribution measured in central neutron probe row. . . . . 1-18

7. Depth variation of initial water content for Scenario IIa, IIb, and IIc. . . . . . . 1-20

8. Wetting and drying moisture characteristic curves used in the hysteretic flow simulation (Scenario IIc). . . . . . . . . . . . . . . . 1-22

9. Spatial variation of $\ln \mathrm{K}(\mathrm{cm} / \mathrm{d})$ for Scenario III . . . . . . . . . . . . 1-23

10. Water content increases at 276 days measured in three neutron probe rows; $y$ value indicates distance from trench face $(\mathrm{cm}) \ldots \ldots \ldots \ldots \ldots \ldots . \ldots \ldots$

11. Comparison of predicted water plumes at $\mathrm{t}=277$ days for Scenarios I, IIa, and III with field results . . . . . . . . . . . . . . . . . . . . 1-29

12. Soil water retention curves used in the Scenario I and II flow modeling . . . . . 1-30

13. Comparison of predicted water plumes at $\mathrm{t}=277$ days for Scenarios IIb and III with field results.

14. Predicted moisture plume at $\mathrm{t}=277$ days for hysteretic case (Scenario IIc) compared with the non-hysteretic simulation (Scenario IIa) and field result. . . . 1-35 
15. Anisotropy ratio as a function of pressure head, $\psi$, predicted from (20) with parameters for the Las Cruces trench site from Polmann et al. (1988) and different ways of evaluating $\mathrm{A}$ and $\sigma_{\mathrm{a}} \ldots \ldots \ldots \ldots \ldots \ldots \ldots \ldots \ldots \ldots \ldots$

16. Moisture plume at $\mathrm{t}=277$ predicted using pressure head dependent anisotropy of the relative permeability. . . . . . . . . . . . . . 1-40

17. Predicted concentration distributions at $\mathbf{t}=\mathbf{2 7 7}$ days for four modeling scenarios

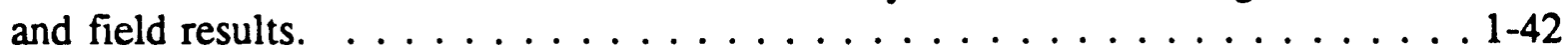

PART 2 APPLICATION OF THE VAM2D COMPUTER CODE FOR MODELING OF FLOW AND TRANSPORT IN FRACTURED MEDIA USING AN EQUIVALENT POROUS MEDIUM APPROACH

2.1. Schematic cross-sectional view of the hypothetical waste disposal site considered in this study. . . . . . . . . . . . . . . . . .2-5

2.2. Fracture network at hypothetical waste disposal site. Note $5 \mathrm{x}$ vertical exaggeration. . .................... . .

2.3. Orientation of the principal axes of anisotropy in a fracture network consisting of two sets of parallel fractures (After Maini and Hocking, 1977; reproduced with permission of the authors). . . . . . . . . . . . . 2-12

2.4. Schematic of parallel fracture system. $\ldots \ldots \ldots \ldots \ldots \ldots \ldots \ldots \ldots \ldots \ldots$ 2-14

2.5. VAM2D finite element grid used in EPM modeling. . . . . . . . . . 2-19

3.1. Boundary conditions and coordinates used in the EPM simulation of steady-state flow. . . . . . . . . . . . . . . . . . . 2-23

3.2. Steady-state hydraulic head distributions in the model domain computed by FRACTRAN (a) and VAM2D (b). . . . . . . . . . . . 2-24

3.3. Boundary and initial conditions used in the VAM2D simulation of conservative and reactive contaminant transport. . . . . . . . . . . . . . 2-27

3.4. Concentration contours of conservative contaminant transport at $t=50$ y with $\phi_{m}$ $=0.05$

3.5. Concentration contours of conservative contaminant transport at $t=150 \mathrm{y}$ with $\phi_{\mathrm{m}}=0.05$ as simulated by FRACTRAN (a) and VAM2D (b). . . . . 2-30 
3.6. Concentration contours of conservative contaminant transport at $t=50 \mathrm{y}$ with $\phi_{\mathrm{m}}$ $=0.25$ as simulated by FRACTRAN (a) and VAM2D (b). . . . . . 2-31

3.7. Concentration contours of conservative contaminant transport at $t=150$ y $\ldots 2-32$

3.8. Concentration contours of reactive contaminant transport at $t=2,500$ y $\ldots \ldots$ 2-36

3.9. Concentration contours of reactive contaminant transport at $i=10,000$ y $\ldots 2-37$ 
Page

PART 1 APPLICATION OF THE VAM2D CODE TO THE SECOND LAS CRUCES TRENCH EXPERIMENT

Table 1. Flow and Transport Parameter Values Used in VAM2D Simulations . . . 1-17

Table 2. Normalized Spatial Moments of Simulated and Observed Water Plumes . 1-36

Table 3. Statistical parameters describing the anisotropic $\mathbf{k}_{\mathrm{r}}(\psi)$ relation.

Parameter values from Polmann et al. (1988). . . . . . . . . . . . . 1-37

Table 4. Normalized Spatial Moments of Simulated and Observed Tritium Plumes . . . . . . . . . . . . . . . . . . . . . . 1-41

PART 2 APPLICATION OF THE VAM2D COMPUTER CODE FOR MODELING OF FLOW AND TRANSPORT IN FRACTURED MEDIA USING AN EQUIVALENT POROUS MEDIUM APPROACH

Table 3.1. Geometric and Hydraulic Fracture Properties of the Modeled System ............................ 2-21

Table 3.2. Porous Medium Properties of the Modeled System . . . . . . . . . . . 2-22

Table 3.3. Effective Hydraulic Parameters Used in the EPM Flow Modeling. . . . . . . . . . . . . . . . . . . 2-22

Table 3.4. Effective Transport Parameters Used in the EPM Modeling of Conservative Contaminant Transport . . . . . . . . . . . . 2-26

Table 3.5. Effective Transport Parameters used in the EPM Modeling of Reactive Contaminant Transport . . . . . . . . . . . . . . 2-35 


\section{ACKNOWLEDGEMENT}

The work described herein was conducted for the U.S. Nuclear Regulatory Commission under contract no. NRC-04-89-090, entitled "Validation and Testing of the VAM2D Computer Code." The support of Project Officer Tom Nicholson throughout the project is gratefully acknowiedged.

The Las Cruces Trench Study (Part 1) benefitted from comments and suggestions made by Dr. Peter Wierenga of the University of Arizona. David Hudson (University of Arizona) provided assistance in re-computing van Genuchten parameters for all soil cores. The computer program used to compute spatial plume moments was provided by Dr. Ed Sudicky of the University of Waterloo. Dr. Sudicky also developed and made available the FRACTRAN discrete fracture flow and transport code used in Part 2 of this report, and provided many valuable comments and suggestions throughout this study. 
PART 1

APPLICATION OF THE VAM2D CODE TO THE SECOND

LAS CRUCES TRENCH EXPERIMENT 


\section{SUMMARY}

The second Las Cruces Trench infiltration experiment was simulated using the VAM2D variably saturated flow and transport code (Huyakorn et al., 1989). The field experiment involves infiltration and redistribution of water and non-reactive tracers in an initially dry, heterogeneous field soil. Intense site characterization and monitoring has been conducted at the Las Cruces field site resulting in a detailed database for calibration and validation of unsaturated flow and transport models. In this study the level of detail in $t^{\prime}$ e flow and transport model required to reproduce key features as well as the significance of hysteresis and saturation dependent anisotropy of the observed water and solute plumes were evaluated. In all cases it was assumed that flow and transport were described by the Richard's and advection-dispersion equation, respectively. However, effects of soil heterogeneity were accounted for in different ways. Four different simulation scenarios were evaluated, i.e.: I) uniform, isotropic soil with literature derived unsaturated hydraulic parameters, IIa) uniform, isotropic soil with unsaturated hydraulic parameters determined by averaging individual sample values, IIb) uniform, but anisotropic soil with unsaturated hydraulic parameters determined in a manner analogous to scenario IIa but different averaging procedure, and III) non-uniform but isotropic soil with different soil material properties assigned to each element in the computational grid in order to represent as closely as possible the heterogeneity observed in the field. The possible effents of hysteresis in the soil moisture characteristic on water movement were evaluated as a variart of scenario IIa.

The field experiment simulated involved infiltration and redistribution of a 75.5 day duration irrigation pulse with conservative tracers (bromide, tritium) supplied during the first 11.5 days. The irrigation was applied at a low rate resulting in unsaturated conditions throughout the experiment. Two-dimensional, cross-sectional simulations were performed using the VAM2D computer code. Even though the experiment was designed to maintain a twodimensional flow regime, the field data showed significant migration in three-dimensions. In order to be able to compare model and field results, the field data were averaged in the third dimension. Salient features of the field moisture content distribution were the irregular water content contours and lateral spreading of the applied water. These features were reproduced quite well in modeling scenario III. Scenario IIb reproduced the horizontal spread of the moisture plume reasonably well, but not the irregular moisture level contours. The simulated effect of hysteresis was the opposite of observed field flow behavior, indicating that capillary hysteresis is not a significant factor in the field experiment. Overall, the results suggest that the observed pattern of water movement at the second Las Cruces trench experiment may be explained either as resulting from local heterogeneities in an isotropic soil, or as resulting from a macroscopic anisotropy in a locally uniform soil. These apparently contradictory results agree qualitatively with the stochastic unsaturated flow theory developed at the Massachusetts Institute of Technology (MIT) by Dr. Lynn Gelhar and coworkers. The latter theory predicts among others, that local soil heterogeneities may manifest themselves in transient unsaturated flow as an apparent large scale anisotropy. It was attempted to model the trench experiment using a pressure head dependent anisotropy factor calculated from the stochastic flow theory. This 
resulted however in a severe overprediction of the degree of anisotropy of hydraulic conductivity, and a poor prediction of water movement patterns.

In addition to unsaturated transient flow, the movement of the tritium tracer which was applied during the first part of the experiment was also simulated with VAM2D, using the four different conceptual flow models discussed above. In contrast to the unsaturated flow results, none of the three modeling scenarios showed good agreement with field concentration data. Maximum observed concentration levels were several times higher than simulated and the bromide plume shape was also not well reproduced. However, field solute monitoring data is much less detailed than available moisture data. It was therefore not possible to resolve whether discrepancies between model transport predictions and field observations are due to deficiencies in the modeling or due to a lack of sufficiently detailed data on three-dimensional solute movement. 


\section{INTRODUCTION}

Modeling of water and chemical novement in the unsaturated zone is a problem with a number of important environmental and agronomic applications. One example is the proper disposal of low-level radioactive waste (LLW). Under the 1980 Low-Level Waste policy Act and Amendments, individual states, as well as groups of states have been seeking to establish new disposal sites. Any new site must meet federal and state regulatory criteria to become licensed, such as 10 CFR Part 61 under the authority of the Nuclear Regulatory Commission. Regulations in 10 CFR Part 61 include the requirement that disposal sites are capable of being modeled. One of the principal reasons that modeling is required is to demonstrate that radionuclide migration away from the disposal waste, either in liquid or gaseous state, will meet regulatory concentration limits at the site boundary or points of exposure. Current regulations require site performance criteria to be satisfied for a period of at least 500 years. Most operating and planned L. W disposal facilities are situated in the unsaturated zone above the water table. The subsurface unsaturated and saturated zones represent one of the primary radionuclide migration and exposure pathways (Shipers, 1989). Problems with existing LLW disposal facilities have often been associated with unfavorable hydrologic conditions at the site (Robertson, 1984).

There is a clear need for accurate and numerically robust subsurface simulation models for use in performance assessments of LLW and other waste disposal facilities. A substantial number of unsaturated and/or saturated zone flow and transport computer models are presently available. Still, the complexity and wide range of conditions encountered at actual sites continue to challenge the capabilities of computer codes and modelers.

The ability to make accurate and reliable predictions may be compromised by limitations of computer codes to account for relevant flow and transport processes, by computational difficulties in solving the governing llow and mass transport equations, and by difficulties in obtaining required model calibration data, particularly in view of the natural heterogeneity of most geologic and soil media. Numerical difficulties can result in long computer execution times and poor mass balance behavior in simulations. Even when comprehensive, numerically accurate and robust simulators are used, incomplete model calibration may prevent accurate prediction of field scale flow and transport behavior. Of these factors, it would seem that alleviating constraints due to limitations of existing computer codes is the easier to achieve, as a result of continued improvement in both compu.er software and hardware. Collecting sufficiently accurate and detailed site characterization required to calibrate a simulation model is likely to remain a tedious, time consuming and expensive proposition. The use of more comprehensive and sophisticated computer codes by itself tends to increase calibration requirements. Even the shear availability of extensive data may not be sufficient to guarantee reliable predictions when the conceptualization of relevant flow and transport mechanisms is inadequate. 
Existing subsurface flow and transport computer models are based almost exclusively on the use of the Richard's equation and advection-dispersion equation to describe variably saturated water flow and solute transport, respectively. In recent years, the influence of spatial heterogeneities in soil properties on field scale flow and transport processes is increasingly recognized. Key flow and transport properties, such as hydraulic conductivity have been found to show large, apparently random, variation even over short distances. As an alternative to classical, deterministic flow and transport models, stochastic theories have been developed which do not seek to describe actual soil conditions at each individual location in a simulation domain, but instead focus on mean flow behavior as influenced by local heterogeneities (Yeh et al., 1982; Mantoglou and Gelhar, 1987). In the stochastic modeling approach the spatial covariance of key model parameters is incorporated into the computational model. The model then predicts mean flow and/or transport behavior and also estimates the uncertainty in the predictions. The stochastic unsaturated flow theory developed by L.W. Gelhar and co-workers (e.g., Polmann et al., 1988) predicts that local soil heterogencities will cause field scale flow behavior that exhibits anisotropy as well as hysteresis. This behavior is qualitatively different from that predicted by conventional deterministic models.

Unfortunately, very few comprehensive and detailed field experiments are available to provide actual data for testing and validating models for field scale flow and transport processes in the unsaturated zone (Russo and Dagan, 1991). The lack of such experiments represents a major impediment to improving the understanding of field scale flow and transport behavior in soils.

During the last several years, ine U.S. Nuclear Regulatory Commission (U.S. NRC) has sponsored detailed field experiments conducted at the University of New Mexico's facility near Las Cruces New Mexico (Wierenga et al., 1986, 1989, 1990). These experiments were designed to study the movement of water containing chemical tracers through an initially dry, spatially variable soil. The series of experiments that have been conducted at this site have involved a very detailed soil characterization and monitoring effort, with the objective of developing a database for testing deterministic as well as stochastic unsaturated flow and transport models. The work described herein presents modeling of the second Las Cruces trench experiment using the VAM2D computer code (Huyakorn, et al., 1989, 1991). VAM2D is a two-dimensional, variably saturated flow and transport code. The flow solution is based on the pressure head form of the Richard's equation, while the transport solution is based on the conventional advection-dispersion equation. One objective of this study was to test the applicability and numerical accuracy of the code for the difficult Las Cruces trench simulation problem. A second objective was to gain more insight in the factors and mechanisms controlling flow and transport in the field experiment. This objective was approached by analyzing a number of different modeling scenarios which differed in the processes that were accounted for in the transient flow simulation. The emphasis in this study is on unsaturated flow modeling on the notion that accurate description of water movement is the first and essential step in assessment of the migration of dissolved chemicals which in many instances will be of ultimate environmental concern. 


\section{MATERIALS AND METHODS}

\subsection{FIELD EXPERIMENT}

A series of unsaturated zone flow and transport field experiments have been conducted at the New Mexico State University's Jornada experimental site near Las Cruces, New Mexico. Detailed descriptions of the site conditions, design of the experiments and experimental data are provided by Wierenga et al. $(1986,1989,1990)$. The following is a summary of site and experimental conditions.

The experimental site is located $40 \mathrm{~km}$ north-east of Las Cruces, New Mexico. The field site is on a basin slope of Mount Summerford, which is part of the Dona Ana mountain range. Climatic conditions, geologic features, geomorphic surfaces, soil types and vegetation in the area are typical of many areas of southern New Mexico and are similar to arid and semi-arid areas of the Southwestern United States. The average annual precipitation is $23 \mathrm{~cm} /$ year while the Class A pan evaporation averages $239 \mathrm{~cm} /$ year. The soil profile at the site shows several buried horizons, indicating a cyclic pattern of sediment accumulation and erosion. Within each of the four main deposits that have been distinguished in the upper $6 \mathrm{~m}$ of the soil, several morphologically different soil horizons have been identified. The sequence of soil layers is depicted in Figure 1. The soil texture in the various horizons shown in Figlire 1 is generally loamy sand or sandy loam.

The present study involves the second trench infiltration experiment conducted at the site. A plan view of the field experiment is shown in Figure 2. The central feature of the experiment is a large trench, $26.5 \mathrm{~m}$ lorg by $4.8 \mathrm{~m}$ wide by $6.0 \mathrm{~m}$ deep which has been dug in the undisturbed soil. The actual experiment was conducted in the area labeled as Plot \#2 in Figure 2. The trench itself is not part of the experimental plot, but it provides access to subsurface monitoring instruments. During trench excavation soil cores and soil samples were taken along the length of the trench at $0.5 \mathrm{~m}$ horizontal intervals. At each sampling location, soil cores were collected at each of the nine sampling depths indicated in Figure 1 to a depth of $5.8 \mathrm{~m}$. A total of 50 cores and samples were collected in this way from each sampling depth. Additional cores were collected at three locations along the trench, using an approximately $20 \mathrm{~cm}$ depth interval, to further resolve vertical soil variation. A total of 640 soil cores and samples were analyzed for determination of bulk density, particle size distribution, saturated hydraulic conductivity and the soil-water retention characteristic.

In addition to the laboratory analyses, saturated hydraulic conductivities were determined in-situ using the bore hole permeameter method. The in-situ hydraulic conductivity measurements were performed for all nine soil layers from which laboratory samples were collected. From each layer, 50 equally spaced measurements along the length of the trench were obtained adjacent to the locations from which samples were collected. 


\section{Morphological Layers}

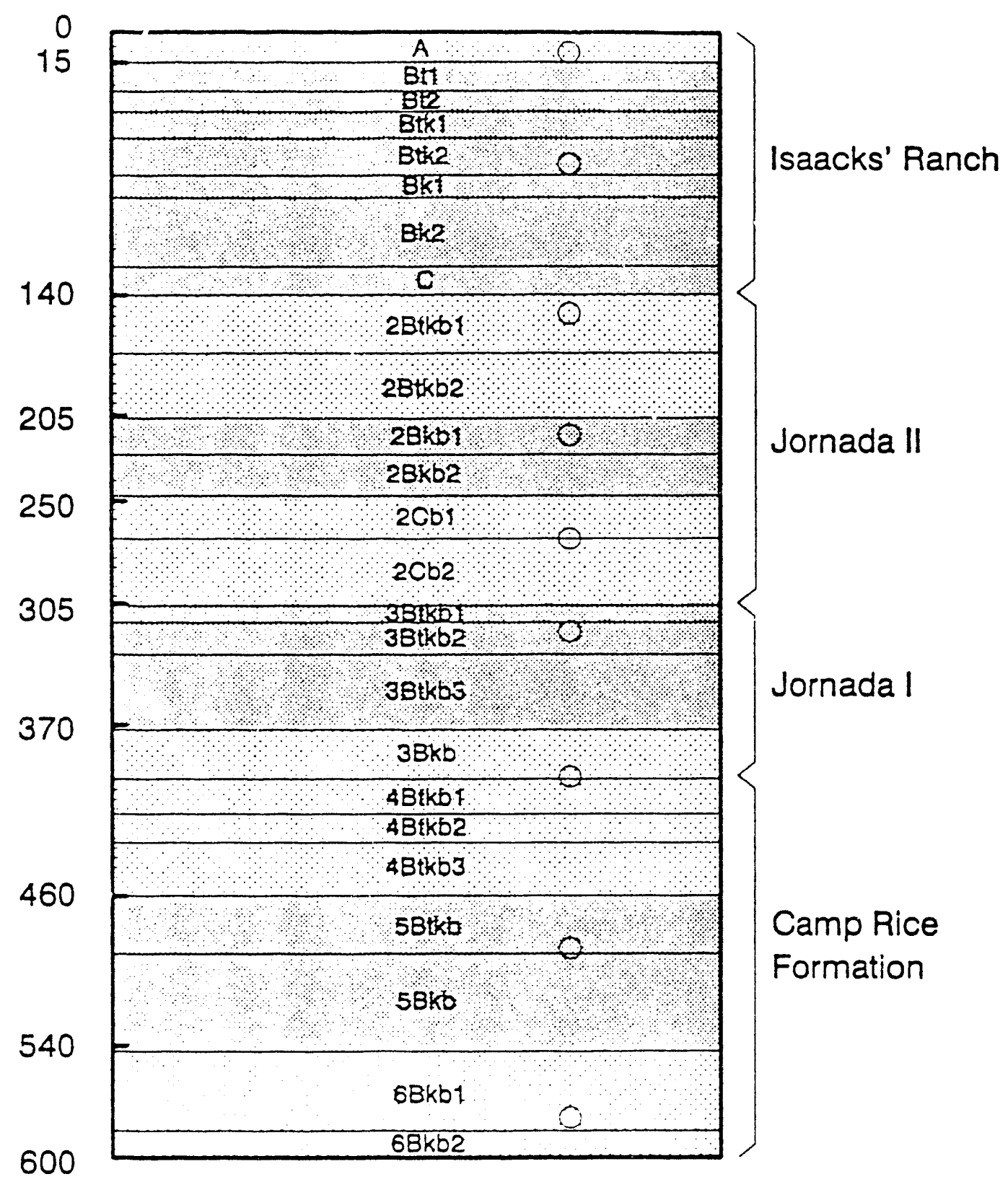

Figure 1. Deposits and soil horizons present in upper $6 \mathrm{~m}$ of the soil at the Jornada experimental site. Circles indicate the depths from which samples were taken (from Wierenga et al., 1989). 


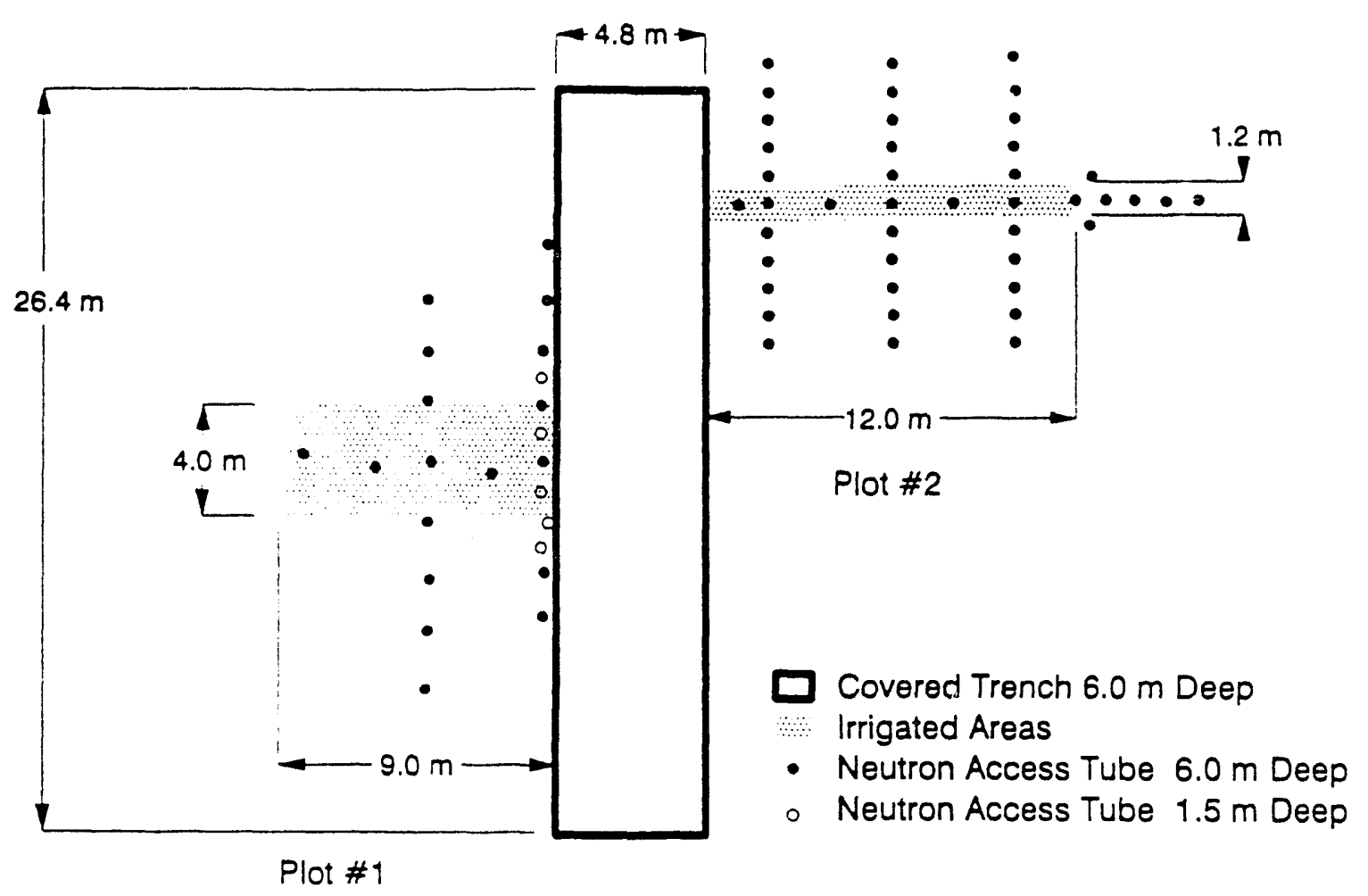

Figure 2. Plan view of the Las Cruces trench site (from Wierenga et al., 1989). 
The infiltration experiment conducted in Plot $\# 2$ on the north side of the trench involved controlled application of water and solute tracers over a $1.22 \mathrm{~m}$ wide by $12 \mathrm{~m}$ long irrigation area, as indicated by shading in Figure 2. Water was applied through a grid of drip emitters, involving a total of 40 parallel irrigation lines. Water was applied twice daily resulting in an average flux density of $0.43 \mathrm{~cm} /$ day over the irrigated area. The application was started on August 8, 1988 and continued for 75.5 days. Tritium and bromide tracers were mixed in with the water during the first 11.5 days. Tritium was added in a concentration of $0.1 \mathrm{mCi} / \mathrm{L}$; the bromide concentration was $0.799 \mathrm{gr} / \mathrm{L}$. The irrigation and surrounding area were covered with a plastic liner throughout the irrigation and subsequent redistribution period, to eliminate both evaporation and infiltration of rain water.

Water flow and solute movement have been monitored during and following the irrigation event using tensiometers, neutron logging and solution samplers. A total of 43 neutron probe access tubes were installed to a depth of $6 \mathrm{~m}$ in the Plot $\# 2$ area. The location of each access tube is shown in Figure 2. Neutron probe readings were taken at $0.25 \mathrm{~m}$ depth increments with a frequency as high as every 4 to 5 days during the irrigation period and decreasing frequency during the distribution period. It may be noted that moisture movement could be monitored in three dimensions $(x, y, z)$ using neutron logging. The tensiometers were installed underneath the center of the irrigation area, through the trench face at an angle of $10^{\circ}$ from horizontal, such that the sensing cups were located in a two-dimensional $(x, z)$ plane located $50 \mathrm{~cm}$ away from the trench face in the $y$-direction. The coordinate convention used here is that the $x$-axis represents the horizontal direction parallel to the long axis of the trench, the y-axis represents the perpendicular horizontal direction, i.e., parallel to the long axis of the irrigation plot, and the z-axis represents the vertical direction. Soil solution samplers were installed from the trench face in a manner similar to the tensiometers. The location of tensiometers and solute samplers underneath the experimental plot is shown in Figure 3. Tensiometers and solution samplers were thus installed in a two-dimensional $x-z$ plane and thus do not provide information on pressure head and concentration variations in the $y$-direction.

\subsection{FLOW AND TRANSPORT MODEL}

\subsubsection{Governing Equations}

The flow solution in the VAM2D computer code is based on the two-dimensional Richard's equation for isothermal flow in a rigid porous medium

$$
\frac{\partial}{\partial x}\left[K_{x x} k_{r} \frac{\partial \psi}{\partial x}\right]+\frac{\partial}{\partial z}\left[K_{z z} k_{r}\left(\frac{\partial \psi}{\partial z}+1\right)\right]=\frac{d \theta}{d \psi} \frac{\partial \psi}{\partial t}
$$

where $\psi$ is pressure head ( $\mathrm{L}), \theta$ is the volumetric water content, $\mathrm{K}_{\mathrm{xx}}$ and $\mathrm{K}_{\mathrm{zz}}$ are the components of saturated conductivity $(\mathrm{L} / \mathrm{T})$ in the $\mathrm{x}$ - and $\mathrm{z}$ - direction, respectively, $\mathrm{k}_{\mathrm{T}}$ is the relative permeability, $x$ and $z$ are cartesian coordinates $(\mathrm{L})$ and $t$ is time $(T)$. We use the convention that 


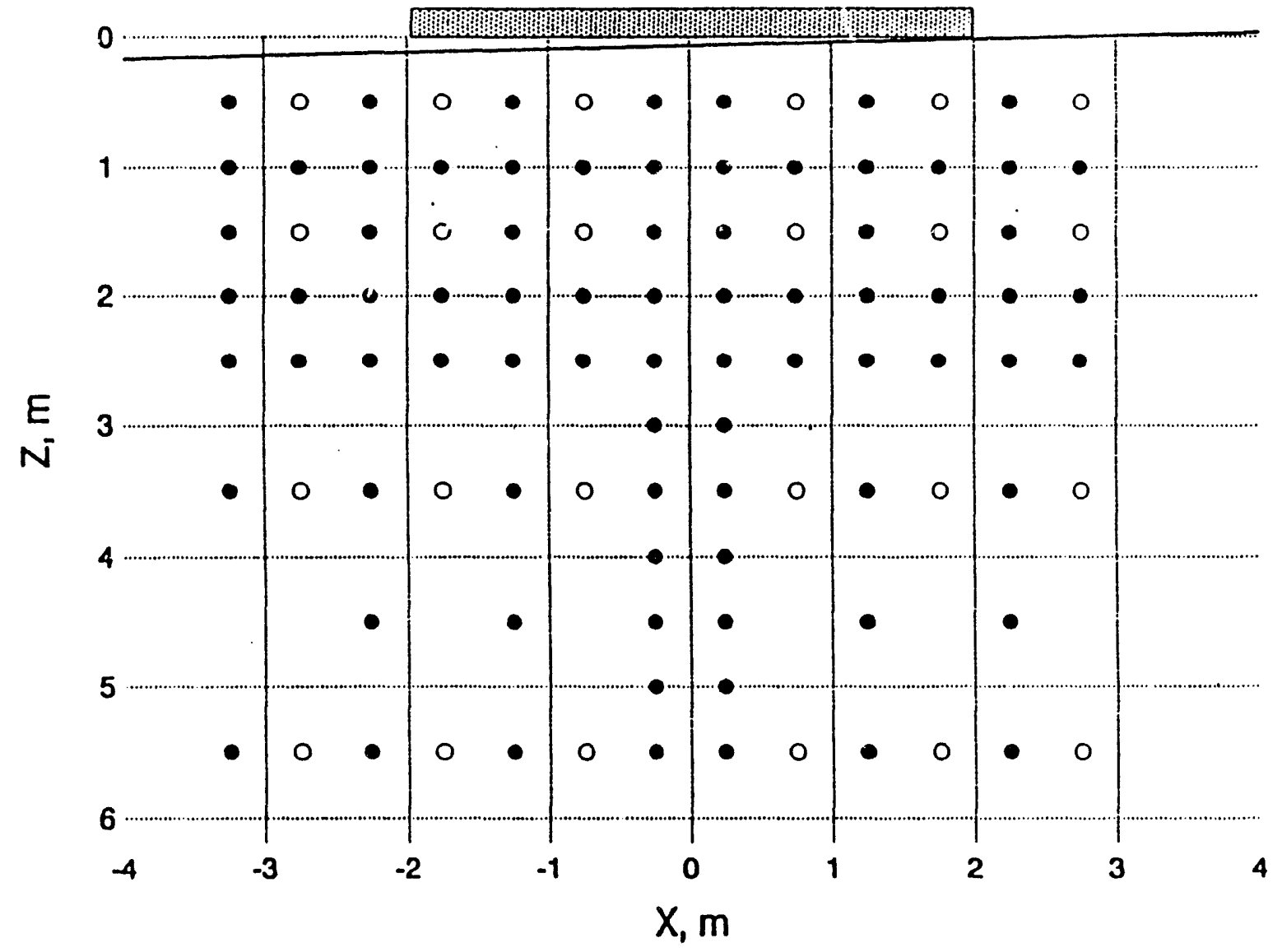

- Tensiometer o Solution Sampler Irigated Area - Soil Surface

Figure 3. Cross-sectional view showing location of tensiometers and solute samplers underneath the irrigation plot (from Wierenga et al., 1990). 
the $z$-coordinate points upward. Constitutive relations used in the flow modeling are taken to be of the form (van Genuchten, 1980)

$$
\begin{gathered}
\theta= \begin{cases}\theta_{s} & \psi \geq 0 \\
\theta_{r}+\left(\theta_{s}-\theta_{r}\right)\left[1+(-\alpha \psi)^{\beta}\right]^{\gamma} & \psi<0\end{cases} \\
k_{r}=S_{e}^{1 / 2}\left[1-\left(1-S_{e}^{1 / \gamma}\right)^{\gamma}\right]^{2}
\end{gathered}
$$

where $\theta_{\mathrm{s}}$ is the saturated water content, $\theta_{\mathrm{r}}$ is a residual water content, and $\alpha, \beta$ and $\gamma$ are shape parameters with $\gamma=1-1 / \beta$. $S_{e}$ is the effective saturation defined as $S_{e}=\left(\theta-\theta_{\mathrm{r}}\right) /\left(\theta_{\mathrm{s}}-\theta_{\mathrm{r}}\right)$.

Transport simulations were based on the advection-dispersion equation for a conservative solute

$$
\begin{gathered}
\frac{\partial}{\partial x}\left(D_{x x} \frac{\partial c}{\partial x}\right)+\frac{\partial}{\partial z}\left(D_{z z} \frac{\partial c}{\partial z}\right)+\frac{\partial}{\partial x}\left(D_{x z} \frac{\partial c}{\partial z}\right)+ \\
\frac{\partial}{\partial z}\left(D_{z x} \frac{\partial c}{\partial x}\right)-V_{x} \frac{\partial c}{\partial x}-V_{z} \frac{\partial c}{\partial z}=\theta \frac{\partial c}{\partial t}
\end{gathered}
$$

where $c$ is solute concentration $\left(M / L^{3}\right), D_{x x}, D_{z z}, D_{x z}$ and $D_{z x}$ are dispersion coefficients $\left(L^{2} / T\right)$ and $V_{x}$ and $V_{z}$ are the $x$ - and $z$-components of the Darcy velocity tensor $(L / T)$. The dispersion coefficients incorporate the effects of hydrodynamic mixing and molecular diffusion and are determined as

$$
\begin{aligned}
& D_{x x}=\alpha_{L} \frac{v_{x}^{2}}{|V|}+\alpha_{v} \frac{V_{Z}^{2}}{|V|}+\tau D^{o} \\
& D_{z z}=\alpha_{v} \frac{V_{x}^{2}}{|V|}+\alpha_{L} \frac{V_{Z}^{2}}{|V|}+\tau D^{o}
\end{aligned}
$$




$$
D_{z x}=D_{x z}=\left(\alpha_{L}-\alpha_{v}\right) \frac{V_{x} V_{z}}{|v|}
$$

where $\alpha_{\mathrm{L}}$ and $\alpha_{\mathrm{v}}$ are the longitudinal and vertical dispersivity (L), respectively, $\mathrm{D}^{\circ}$ is the bulk solution coefficient of molecular diffusion $\left(\mathrm{L}^{2} / \mathrm{T}\right)$ and $\tau$ is a water content dependent tortuosity coefficient given by (Millington and Quirk, 1959)

Using the tortuosity factor given by (6) has the effect of reducing molecular diffusion as the water content decreases. It was found necessary to introduce this tortuosity factor after initial simulations with a constant effective diffusion coefficient indicated a significant overprediction of diffusion.

$$
\tau=\theta^{10 / 3} \theta_{s}^{-2}
$$

The flow equation is solved in VAM12D using the Galerkin finite element technique with either Picard or Newton-Raphson iteration methods. The transport equation is treated using the upstream-weighted finite element method. The transport solution in VAM2D can account for linear equilibrium sorption as well as (chained) decay reactions. However, these processes were ignored in the present analysis. For bo hl now and transport problems, spatial discretization is performed using simple rectangular elements or orthogonal curvilinear elements, allowing element matrices to be computed using highly efficient influence coefficient matrix formulas. The numerical schemes used are well docuriented (Huyakorn and Pinder, 1983; Huyakorn et al., 1984, 1985, 1989) and are not repeated here. Various aspects of the computational procedure that are related specifically 10 mass balance accuracy and convergence of the transient, variably saturated flow solution are discussed in the next section.

\subsubsection{Numerical Procedures for Efticienı Flow and Transport Simulation}

Simulation of transient water sird solute movement in very dry soils represents a numerically challenging problems. Fiperience with pressure head based solutions of the Richards equation has shown that it may be difficult to obtain a solution with accurate mass balance, unless very fine space and time discretizations are employed (e.g. Hills et al., 1989). Typically it is found that mass is "lost" in the simulation and that computed infiltration depths are less than they should be. Mass balance errors in numerical solutions of (1) are usually related to the manner in which the storage terms in the governing equaticn are treated. Although effective ways to treat this problem have been known for some time (e.g. Cooley, 1983, Hornung and Messing, 1984) difficulties that have been encountered in modeling of the Las Cruces trench experiments have caused renewed interest in the mass balance problem in unsaturated flow modeling (Hills et al., 1989; Celia et al., 1990). 
Milly (1985) has discussed several alternative ways to evaluate the moisture capacity $\mathrm{C}=\mathrm{d} \theta / \mathrm{d} \psi$ in finite element solutions of (1) that can be used to achieve an accurate moisture mass balance. The usual definition of the moisture capacity as the tangential approximation of $\mathrm{d} \theta / \mathrm{d} \psi$ will result in an exact mass balance only for infinitely small space and time discretizations. For more practical grid sizes and time steps, substantial mass balance errors may occur when sharp moisture fronts are present. In order to obtain a mass conservative result, Milly (1985) pointed out that the moisture capacity should be defined in such a way that the following holds at the element level

$$
\int_{e} \bar{C}\left(\psi^{t+\Delta t}-\psi^{t}\right) d e=\int_{e}\left(\theta^{t+\Delta t}-\theta^{t}\right) d e
$$

in which $\bar{C}$ is the effective soil water capacity expressed in terms of the finite element interpolation functions within the element and where integration is performed on an element by element basis. The resulting expression for the nodal values of $\mathrm{C}$ depend on how the finite elemunt storage matrix is formed, i.e., whether or not a diagonalized (lumped) matrix is used. The simplest scheme results when mass-lumping is applied so that the element storage matrix is the same as in finite difference schemes, i.e., a diagonal matrix given by

$$
[C]^{e}=\frac{\Delta_{x} \cdot \Delta_{y}}{4 \Delta t}\left[\begin{array}{lll}
C_{1} & & 0 \\
& \ddots & \\
0 & & C_{4}
\end{array}\right]
$$

where $\Delta_{x}$ and $\Delta_{y}$ are the element dimensions. In this case the nodal values of $C_{i}$ are given simply by

$$
C_{i}^{t+\Delta t}=\frac{\theta_{i}^{t+\Delta t}-\theta_{i}^{t}}{\psi_{i}^{t+\Delta t}-\psi_{i}^{t}}
$$

The same result has been presented by Horning and Messing (1984). Celia et al. (1990) have recently reviewed an alternative approach, based on the mixed form of the Richards equation which yields an equivalent result. In the VAM2D simulations a mass-lumped flow solution was used with a backward difference time approximation and with nodal moisture capacities evaluated using (9). The average cumulative mass balance errors expressed as a fraction of the total added amount of water was $1.2 \%$ for all flow simulations. In contrast, initial simulations which used a non-mass conserving way to evaluate moisture capacities showed mass balance errors as high as $20 \%$. The mass balance error could presumably be reduced further by using a finer finite element grid and smaller time steps. However, it was felt that the level of accuracy achieved in the flow simulations was commensurate with the level of detail in available field data. 
While mass balance accuracy of a numerical unsaturated flow solution can be quite sensitive to the manner in which storage terms are calculated, the mass balance error in solute transport solutions can be similarly sensitive to the way in which the velocity components $\left(V_{x}\right.$, $V_{z}$ ) are computed. When saturation and hydraulic conductivity values vary drastically across an element, it is intuitively clear that transport solutions can be sensitive to the nodal averaging procedures used to calculate elemental velocity values. Velocities are obtained by local application of Darcy's Law which requires knowledge of the effective elemental hydraulic conductivity. The computationally most expedient way to calculate these is to evaluate element water content and relative conductivities after averaging nodal pressure head values, i.e.,

$$
\begin{aligned}
& \langle\psi\rangle=\frac{1}{n} \sum_{j=1}^{n} \psi_{j} \\
& \langle\theta\rangle=\theta(<\psi>) \\
& \left.<k_{r}\right\rangle=k_{r}(<\theta>)
\end{aligned}
$$

where $<>$ denotes elemental value, $\mathrm{k}_{\mathrm{r}}$ is the relative permeability and $\mathrm{n}$ is the number of nodes associated with the element. In this case the constitutive relations $\theta(\psi)$ and $\mathrm{k}_{\mathrm{r}}(\theta)$ need to be evaluated only once for each element. However, in the transport simulations of the trench experiment, the above scheme resulted in solute mass balance errors as high as $10 \%$, even though very good mass balance was obtained in the flow solution itself. It was found that solute mass balance errors could effectively be eliminated by performing the averaging over the nodal values of $\theta$ and $\mathrm{K}_{\mathrm{r}}$ themselves as

$$
<\theta>=\frac{1}{n} \sum_{j=1}^{n} \theta\left(\psi_{j}\right)
$$

and

$$
<k_{r}>=\frac{1}{n} \sum_{j=1}^{n} k_{r}\left(\theta_{j}\right)
$$

In the flow and transport simulations, the bulk of the computational effort is taken up by the nonlinear flow solution, and a considerable savings can be achieved by using efficient time stepping procedures in conjunction with techniques to enhance convergence of the iterative solution scheme. VAM2D incorporates several of these features and the trench simulations provided a good test for their effectiveness. The features in VAM2D are not entirely new, rather they are based on techniques that have been found to be effective elsewhere (e.g. Cooley, 
1983; van Genuchten, 1982; Huyakorn et al., 1984). Mass-lumping and use of a fully implicit time approximation are well known to reduce oscillations and thus imprc ve convergence (e.g. Milly, 1985; Celia et al,, 1990). Use of a Newton-Raphson iteration procedure will also improve convergence compared to the Picard scheme. However, the Newton-Raphson procedure itself requires approximately twice the effort per iteration that the Picard scheme does, so the trade-off between Picard and Newton-Raphson iteration is not always clear. In the VAM2D trench simulations, Picard iteration was used in conjunction with an under-relaxation scheme for updating nodal pressure head values between iterations, based on a procedure proposed by Cooley (1983). This combination was found to be quite competitive in speed of convergence with Newton-Raphson iteration while requiring less computational effort. Another aspect of the computational scheme is the selection of optimal time step size during the transient simulation. Aside from the effect that time step size has on the simulation accuracy, there will be a large effect on CPU time. An overly small timestep may slow the simulation down, even though the solution will probably converge rapidly ai each time step. On the other hand, convergence may be very slow or fail at all if a toc large timestep is used, necessitating cutbacks in time step size and/or a large number of iterations per time step. VAM2D incorporates a simple automatic time step adjustment scheme, adopted from van Genuchten (1982), which has proven very effective. In this scheme an initial, minimum and maximum time step size are specified as input parameters. The simulation is started using the initial time step size. At the beginning of each new time step, the step size is adjusted depending on the number of iterations required during the just completed time step. If convergence was rapid, the time step size is increased by a preset fraction until the maximum allowable stepsize is reached, otherwise the step size is kept constant or, if convergence was slow, decreased. The minimum time step value guards against excessively long simulation times if convergence is slow. The maximum step size is set to control errors in the numerical approximation. In the Las Cruces trench simulations the initial time step was set to $\Delta t_{\text {in }}=1$ day and the maximum time step was set to $\Delta t_{\max }=7$ days. All simulations discussed in this report covered a 277 day time period; between 90 and 130 time steps were required for the transient simulations.

A final consideration is the selection of an appropriate pressure head convergence tolerance. In the relatively wet parts of the modeled domain, a small convergence tolerance should be used, since water contents and conductivities will be sensitive to relatively small pressure head changes. On the other hand, in the dry parts of the domain, the water contents approach residual values and are quite insensitive to pressure head changes. Consequently, a much larger convergence tolerance can be used here. In order to accommodate both considerations, we used a combination of absolute and relative tolerances. The nodal convergence tolerance, $\tau_{\mathrm{i}}$, is then determined from

$$
\tau_{i}=\tau_{a}+\tau_{r}\left|\psi_{i}\right|
$$

where $\tau_{\mathrm{a}}$ and $\tau_{\mathrm{r}}$ are the absolute and relative tolerances and where $\tau_{\mathrm{r}}$ typically ranges from 0.01 to 0.001 . 
All simulations reported here were performed on a $25 \mathrm{MHz} 80386$ personal computer equipped with a 80387 FPU and 8 megabytes of RAM memory. The flow and transport simulations required between 4 (Scenario I and II) and 7 (Scenario III) hours of computer time. While these simulation times are by no means trivial, they compare quite favorably with results reported by Hills et al. (1989) which demonstrates the effectiveness of the computational schemes employed in the VAM2D computer code.

\subsubsection{Model Domain and Boundary Conditions}

Flow and transport were modeled in a two-dimensional vertical ( $x-z$ plane) cross-section. Flow and transport in the $y$-direction were not considered. Restricting the modeling to two dimensions is dictated by the selection of the 2-D VAM2D computer code. However, considering the relatively large dimension of the irrigation area in the $y$-direction (Figure 2) and the uniform water and tracer applications, this assumption was deemed reasonable for most of the actual plot, with possible exception of the ends of the plot. The modeled domain and assigned boundary conditions are depicted in Figure 4. This figure also shows the spatial coordinate convention used in the modeling. The $x$-coordinate origin is taken to be directly underneath the center of the experimental plot. The modeled region extends $600 \mathrm{~cm}$ to both the left and right. The vertical (z-) coordinate is taken to be positive upwards. The soil surface is thus located at an elevation of $500 \mathrm{~cm}$. The dimensions of the modeled region were chosen large enough that boundary effects on flow and transport would be negligible. As shown in Figure 4, the left and right side boundaries are assigned no-flux conditions for flow and transport. The upper boundary which corresponds to the soil surface is also assigned a no-flux condition, except for the zone that corresponds to the irrigation area. This boundary section was assigned time-dependent water and solute flux conditions corresponding to the water and solute applications in the field experiment. The lower boundary was assigned a zero pressure head gradient and zero concentration gradient condition. These conditions will allow the free-exit of water and solute through the boundary, and are a reasonable representation of physical conditions in a deep, well-drained soil.

The finite element discretization of the model domain is shown in Figure 5. A total of 1508 rectangular elements was used, with the element size varying from $10 \mathrm{x} 10 \mathrm{~cm}$ underneath the source to $30 \times 30 \mathrm{~cm}$ near the side and lower boundaries. The chosen discretization provided sufficient resolution in the flow and transport simulations without requiring excessive

computational effort. Initial conditions and assignment of material zones were different for the various simulation scenarios considcred, and are discussed below.

\subsubsection{Flow and Transport Modeling Scenarios}

An important aspect of the conceptualization underlying the flow and transport modeling was that soil hydraulic properties and their spatial distribution have a pronounced influence on flow and solute transport behavior. We therefore evaluated different simulation scenarios that 
employed varying amounts and detail of site specific hydraulic data for model calibration. Alternatively, these different simulation scenarios may be thought of as representing different conceptual. indels of the key factors controlling flow and solute transport of the trench site.

The different simulation scenarios/conceptual models were:

I. Uniform, isotropic soil with hydraulic properties of a typical sandy loam soil. This scenario used no site specific data for flow calibration.

IIa. Uniform isotropic soil with hydraulic properties given by averages obtained from trench soil core data.

IIb. Uniform, but anisotropic soil with soil hydraulic properties determined as in Scenario IIa, with exception of saturated hydraulic conductivity which was allowed to be anisotropic and calculated assuming a lognormal distribution of local $\mathrm{K}$ values.

IIc. As Scenario IIa, except that the effect of hysteresis in the moisture retention characteristic is accounted for in the computer simulation.

III. Non-uniform, isotropic soil with spatial variation of hydraulic properties and initial conditions reflecting as close as possible that obtained from soil core samples and neutron logging, respectively.

In each of the above scenarios, the initial condition for the flow simulations was defined in terms of moisture content(s) as measured at the site prior to the experiment. In order to convert these to initial pressure head(s) which are required for the VAM2D computer code, the inverse of (2) was used with appropriate values of the parameters $\theta_{\mathrm{s}}, \theta_{\mathrm{r}}, \alpha$ and $\beta$. It should be noted that the individual soil core values of $\partial_{\mathrm{r}}, \alpha$ and $\beta$ used for Scenarios II and III are different from the values given in the Las Cruces trench database (Wierenga et al., 1989). For a number of the soil cores, the $\theta_{\mathrm{r}}$ values given in the database are larger than actual water contents measured in the field at the same $x$ - and $z$-locations, prior to the initiation of water and solute application. This would imply that the initial pressure head at these locations was undefined. In reality the soil cores, which were collected inside the trench, and neutron probe measurements represent data from spatially different locations, i.e., different y-coordinate (see Figure 2) and the conflict between $\theta_{\mathrm{r}}$ values in the database and actual measurements of water contents may be an artifact of modeling the site as a two-dimensional cross-section in which soil variations in the $y$-direction are ignored. On the other hand, the parameter $\theta_{\mathrm{r}}$ in (2) is essentially a curvefitting parameter and there is no physical objection to imposing certain constraints in the parameter estimation. A new set of values of $\theta_{\mathrm{r}}, \alpha$ and $\beta$ was therefore determined for each soil core using the following procedure: The soil layer from which each core was collected was determined. An upper bound for $\theta_{\mathrm{r}}$ was determined for each of the nine identified soil layers as slightly less than the minimum observed water content for that layer. $\theta_{\mathrm{r}}, \alpha$ and $\beta$ were 


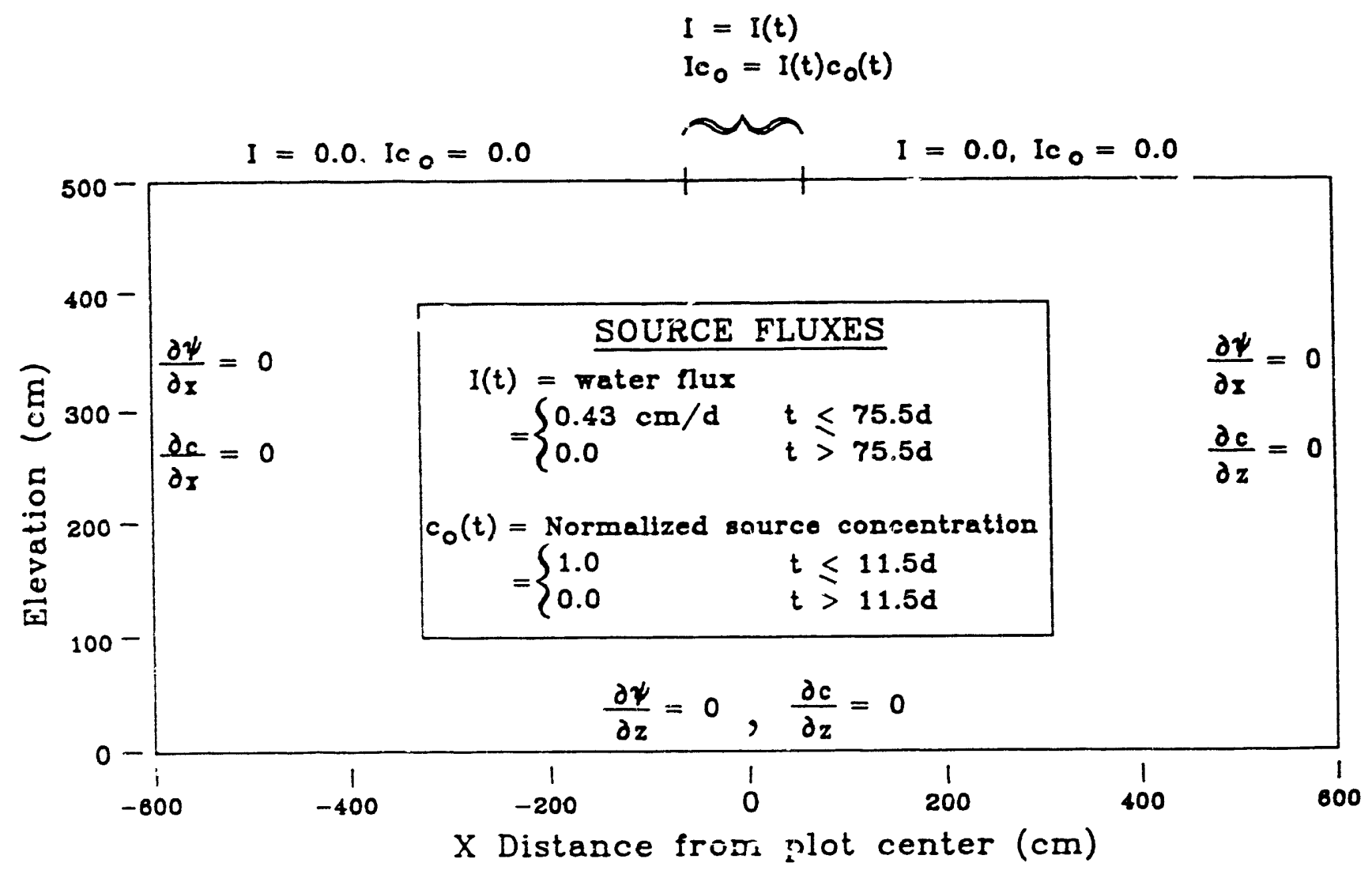

Figure 4. Schematic view of the modeled two-dimensional cross-section and assignment of boundary conditions. 


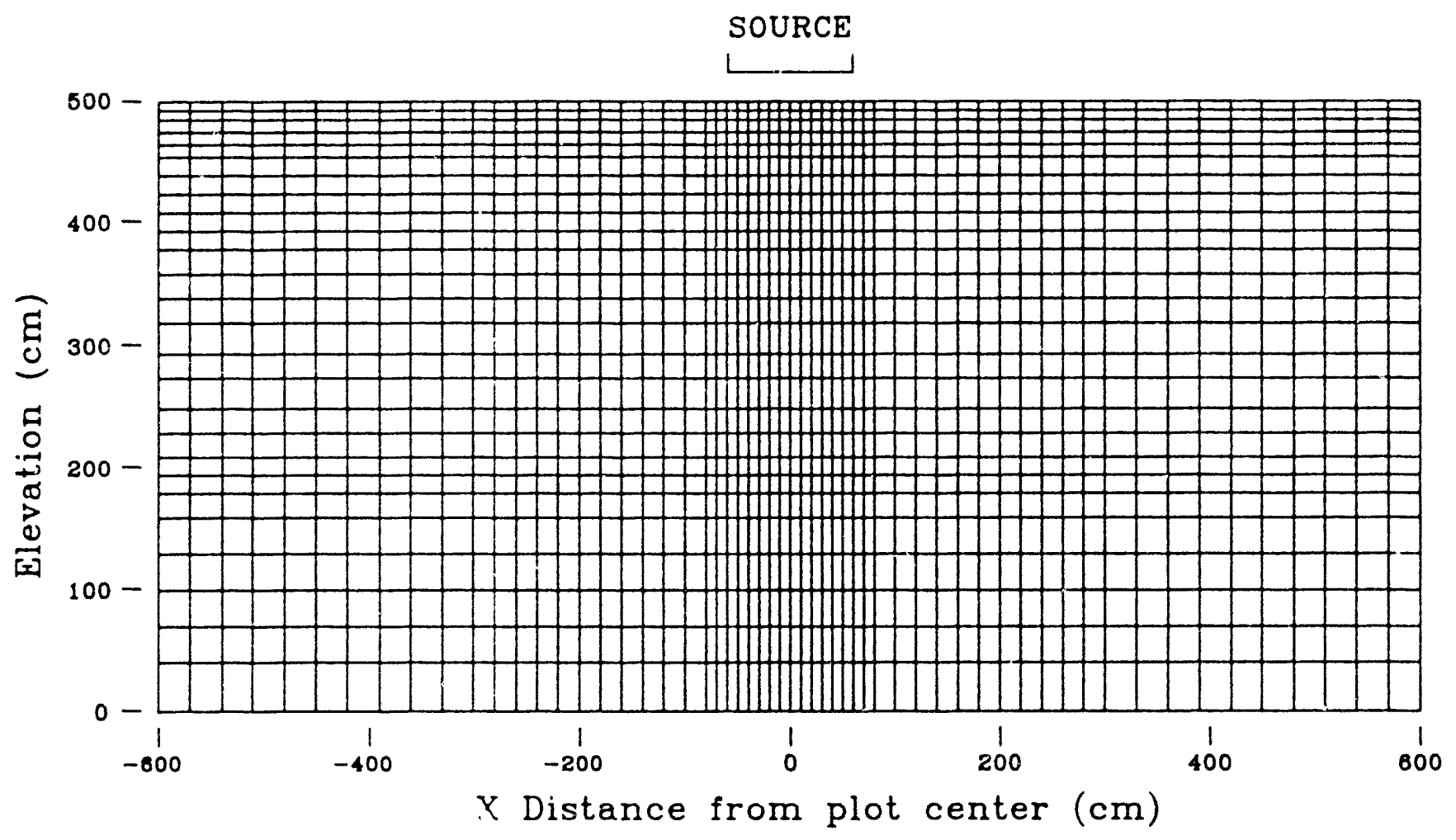

Figure 5. Finite element discretization used in VAM2D flow and transport simulations. 
subsequently fitted again to the experimental $\theta(\psi)$ curves under this constraint. The resulting van Genuchten parameters for each core sample used in the VAM2D modeling are listed in Appendix A. The rationale and input parameter selection for each of the modeling scenarios are discussed below.

\subsubsection{Scenario I}

In this scenario it was assumed that very little site specific data was available for model calibration. This case corresponds to the situation, in practice not at all uncommon, where computer modeling is performed while little or no site characterization data is available and one has to estimate key model parameters. Since for the Las Cruces site there actually is quite a large amount of data available, it is possible to assess the accuracy of model predictions obtained with estimated parameters. This scenario represents a base case for evaluating the gain in accuracy obtained with the other modeling scenarios which do use site specific information. The soil in Scenario I was assumed to be uniform and isotropic. The only site specific information used was the soil textural classification (sandy loam) and an estimate of the initial water content. Estimated van Genuchten soil parameters for the sandy loam soil were obtained from the literature (Carsel and Parrish, 1988; see Table 1). The initial water content distribution was taken to be uniform and assigned a value of $\theta_{\mathrm{i}}(\mathrm{x}, \mathrm{z})=0.10$, which corresponds approximately to the actual average water content in the soil at the initiation of irrigation.

Table 1. Flow and Transport Parameter Values Used in VAM2D Simulations

\begin{tabular}{|c|c|c|c|c|c|}
\hline & \multicolumn{5}{|c|}{ Modeling Scenario } \\
\hline & I & IIa & Ilb & IIc & III \\
\hline \multicolumn{6}{|c|}{ Hydraulic Parameters } \\
\hline $\begin{array}{l}\mathrm{K}_{\mathrm{xx}} ; \mathrm{K}_{\mathrm{zz}}(\mathrm{cm} / \mathrm{d}) \\
\theta_{\mathrm{g}} \\
\theta_{\mathrm{r}} \\
\alpha\left(\mathrm{cm}^{-1}\right) \\
\beta\end{array}$ & $\begin{array}{l}106 ; 106 \\
0.410 \\
0.065 \\
0.075\end{array}$ & $\begin{array}{l}270 ; 270 \\
0.320 \\
0.025 \\
0.112\end{array}$ & $\begin{array}{l}1310 ; 655 \\
0.320 \\
0.025 \\
0.112\end{array}$ & $\begin{array}{l}270 ; 270 \\
0.320 \\
0.025 \\
0.112 ; 0.224\end{array}$ & $\begin{array}{l}\text { individually } \\
\text { assigned to } \\
\text { every element }\end{array}$ \\
\hline \multicolumn{6}{|c|}{ Transport Parameters } \\
\hline $\begin{array}{l}\alpha_{\mathrm{L}}(\mathrm{cm}) \\
\alpha_{\mathrm{T}}(\mathrm{cm}) \\
D^{\circ}\left(\mathrm{cm}^{2} / \mathrm{d}\right)\end{array}$ & $\begin{array}{l}5.0 \\
5.0 \\
1.0\end{array}$ & $\begin{array}{l}5.0 \\
5.0 \\
1.0\end{array}$ & $\begin{array}{l}5.0 \\
5.0 \\
1.0\end{array}$ & $\begin{array}{l}- \\
-\end{array}$ & $\begin{array}{l}5.0 \\
5.0 \\
1.0\end{array}$ \\
\hline
\end{tabular}




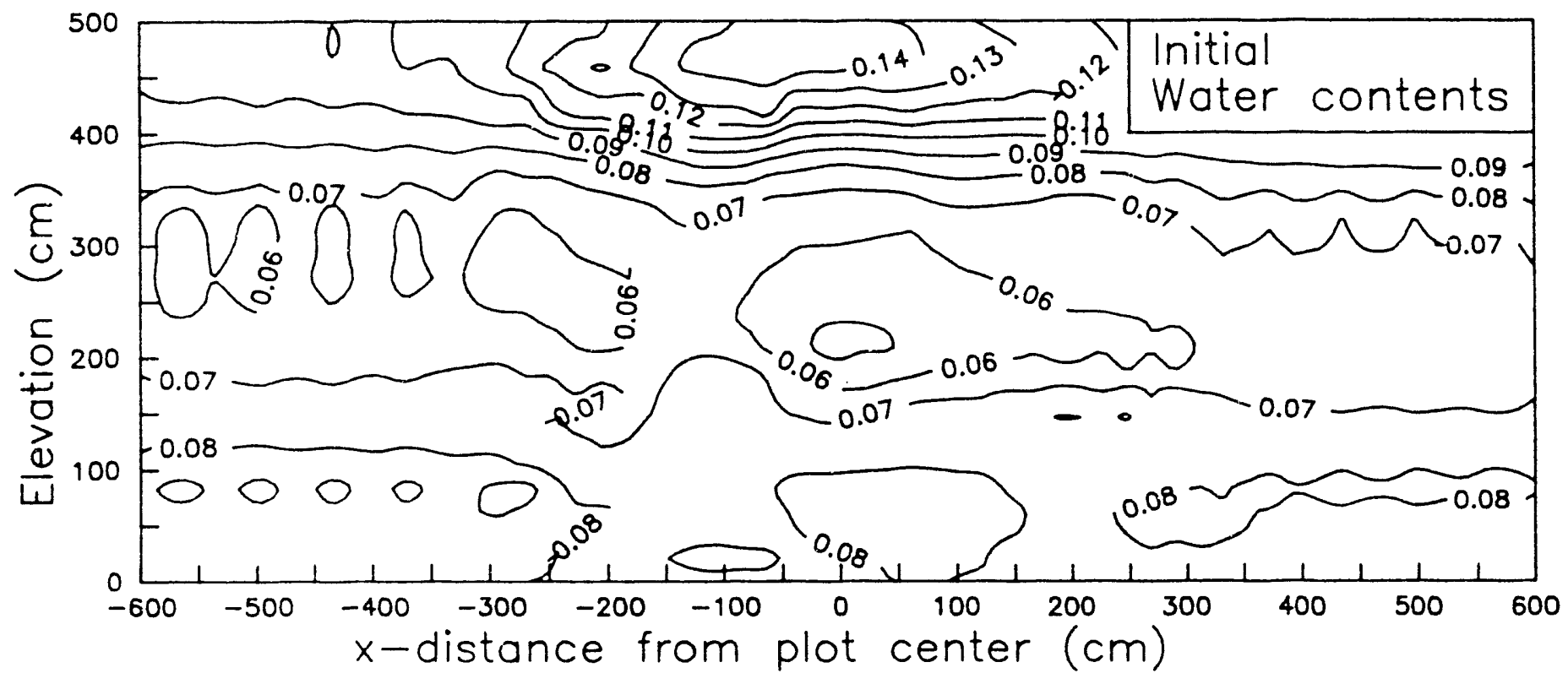

Figure 6. Initial water content distribution measured in central neutron probe row. 


\subsubsection{Scenario IIa}

In this scenario, the soil was again taken to be uniform, but it was assumed that sufficient site characterization data were available to accurately define average values of the van Genuchten parameters. The values (Table 1) used are the averages of the parameters for the individual soil cores. This modeling scenario tests the assumptioin that any effects of local soil heterogeneity are averaged out at the scale of the field experiment, and that the experiment can thus be reproduced using averaged soil properties. The average values of parameters $\theta_{\mathrm{r}}, \theta_{\mathrm{s}}$, and $\alpha$ and $\beta$ were calculated as arithmetic means of individual soil core values, while saturated conductivity was calculated as a geometric mean of individual soil core values (Wierenga et al., 1989). The initial water content distribution in Scenario Ila was obtained from field neutron probe readings taken just prior to the water and solute application. Data from the central row of neutron probe access tubes (Figure 2) were used. The field data (Figure 6) show a distinct variation of water content with depth, but more or less uniform water contents in the $\mathrm{x}$-direction. The vertical variation of water contents was retained in the simulation, but a uniform water content in the $x$ direction was used, resulting in the modeled initial water content vertical distribution shown in Figure 7.

\subsubsection{Scenario IIb}

Scenario IIb is a variant of Ila; it was included in the simulation following evaluation of Scenario IIa, in order to improve the agreement between the VAM2D simulation and observed water content distributions following infiltration and redistribution. Scenario IIb differs from IIa in the way saturated hydraulic conductivity is evaluated. In Scenario IIb, the average hydraulic conductivity was computed assuming a lognormal distribution of individual K-values, i.e.,

$$
<K>=\exp \left(\mu_{\ln }+1 / 2 \sigma_{\ln }^{2}\right)
$$

where $\mu_{\ln }$ and $\sigma_{\ln }$ are the mean and standard deviation, respectively, of the log transformed $\mathrm{K}$ values of individual soil cores and angular brackets denote mean value. Additionally, the saturated hydraulic conductivity in this scenario was taken to be anisotropic with

$$
<K_{x x}>=2<K_{z z}>
$$

Since all soil cores were taken vertically downward, measured K-values represent vertical conductivities; the mean conductivity calculated from (7) thus estimates $\mathrm{K}_{\mathrm{zz}}$. The horizontal conductivity in Scerario IJb was simply assigned a value twice as large as the vertical value. Values of the hydraulic parameters used in this scenario are listed in Table 1. 


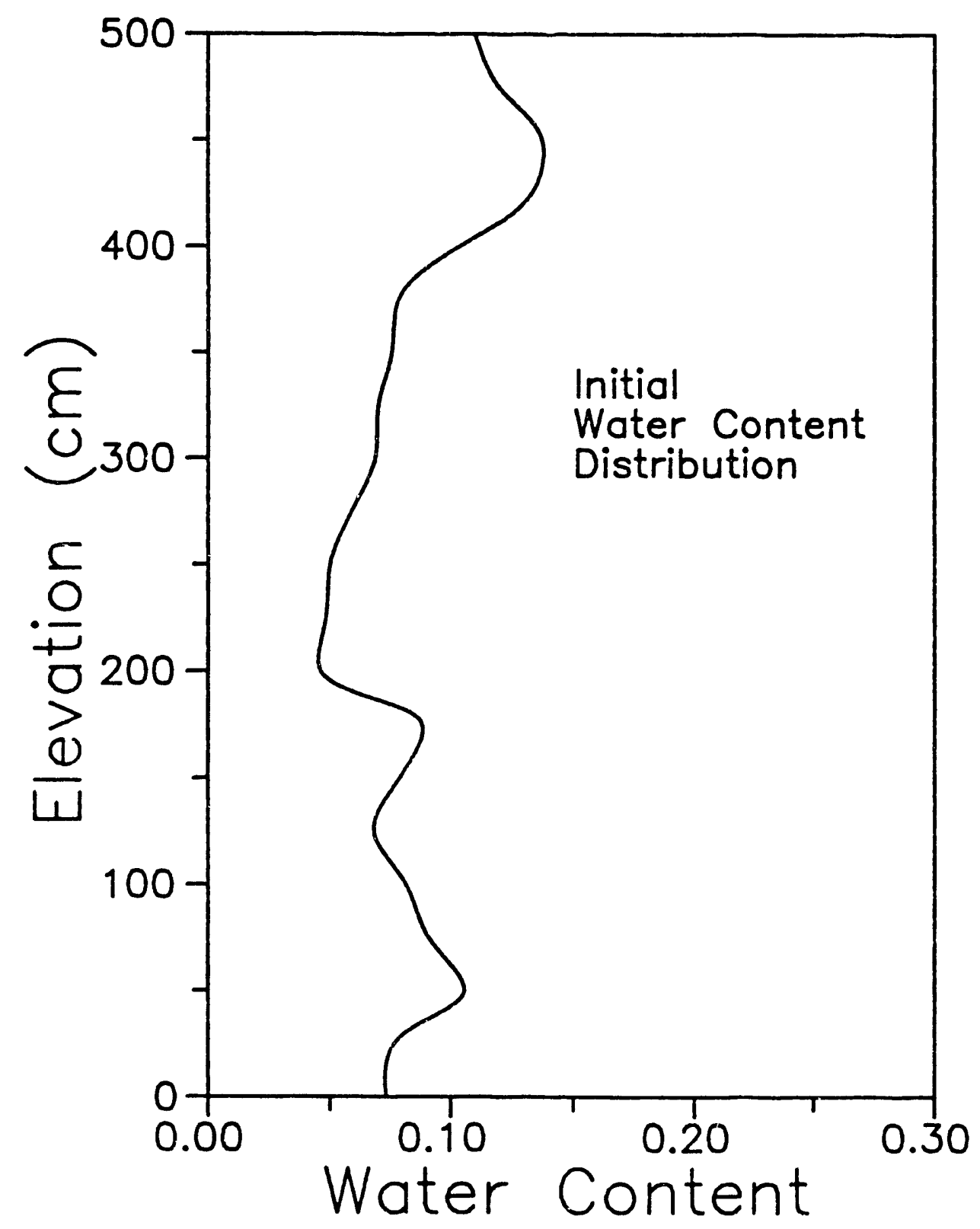

Figure 7. Depth variation of initial water content for Scenario IIa, IIb, and IIc. 


\subsubsection{Scenario IIc}

This simulation was another variant of the second modeling scenario, designed to evaluate the possible influence of soil moisture hysteresis on infiltration and distribution of water. The VAM2D computer code has the ability to model hysteresis in the $\theta(\psi)$ relation, using the procedure described by Kool and Parker (1987). The general procedure for modeling hysteresis requires that soil moisture parameters describing both the drying and wetting branches of the soil moisture characteristic are specified. The code then keeps track of the state, either wetting or drying, as well as the appropriate $\theta(\psi)$ scanning curve of each element in the computational grid during the simulation, and evaluates the hydraulic properties accordingly. No actual data on soil moisture hysteresis are available for the trench site. Measured $\theta(\psi)$ relations all correspond to the main drying branch of the moisture characteristic. Lacking data on the wetting characteristic, the same average moisture parameter values were used for both wetting and drying branches, except that the $\alpha$ parameter for the wetting branch was assigned a value of twice the drying branch value, as recommended by Kool and Parker (1987). The resulting hysteretic moisture retention characteristic is shown graphically in Figure 8. It can be seen that the assumed ratio of $\alpha^{\mathrm{w}}: \alpha^{\mathrm{d}}=2.0$, imposes only a modest degree of hysteresis. However, the ratio used is typical of undisturbed field soils (Kool and Parker, 1987).

\subsubsection{Scenario III}

In the final simulation scenario, all available data were utilized to determine spatially variable soil hydraulic properties and initial water contents. Material properties and initial water contents were allowed to vary from element to element. The van Genuchten parameter values describing hydraulic relationships at element centroids of the finite element grid were determined by spatially interpolating values from soil cores collected in a two-dimensional ( $x-z)$ grid inside the trench. Initial water contents were determined similarly from field neutron probe readings taken just prior to the field experiment. Measurements from the middle neutron probe row were used. Spatial interpolation was achieved by kriging. In the case of saturated hydraulic conductivity, the interpolation was performed on ln-transformed values. The resulting distribution of saturated conductivity is shown as an illustrative example in Figure 9. Although different material properties were assigned to each element in the computational grid, these hydraulic parameter values were determined from samples taken several meters away from the location of the actual infiltration experiment. It can thus not be expected that the elemental soil properties result in good point-wise correspondence with actual soil properties. However, invoking the assumption of ergodicity (Dagan, 1990), it is expected that the spatial variability of soil properties is reproduced in Scenario III, allowing a direct evaluation of the effects of local soil heterogeneity on simulated flow and transport behavior.

\subsubsection{Solute Transport Parameters}

Tritium and bromide were added as tracers during the first part of the irrigation experiment. Both tracers would be expected to behave as ideal, non-reactive tracers. Field 


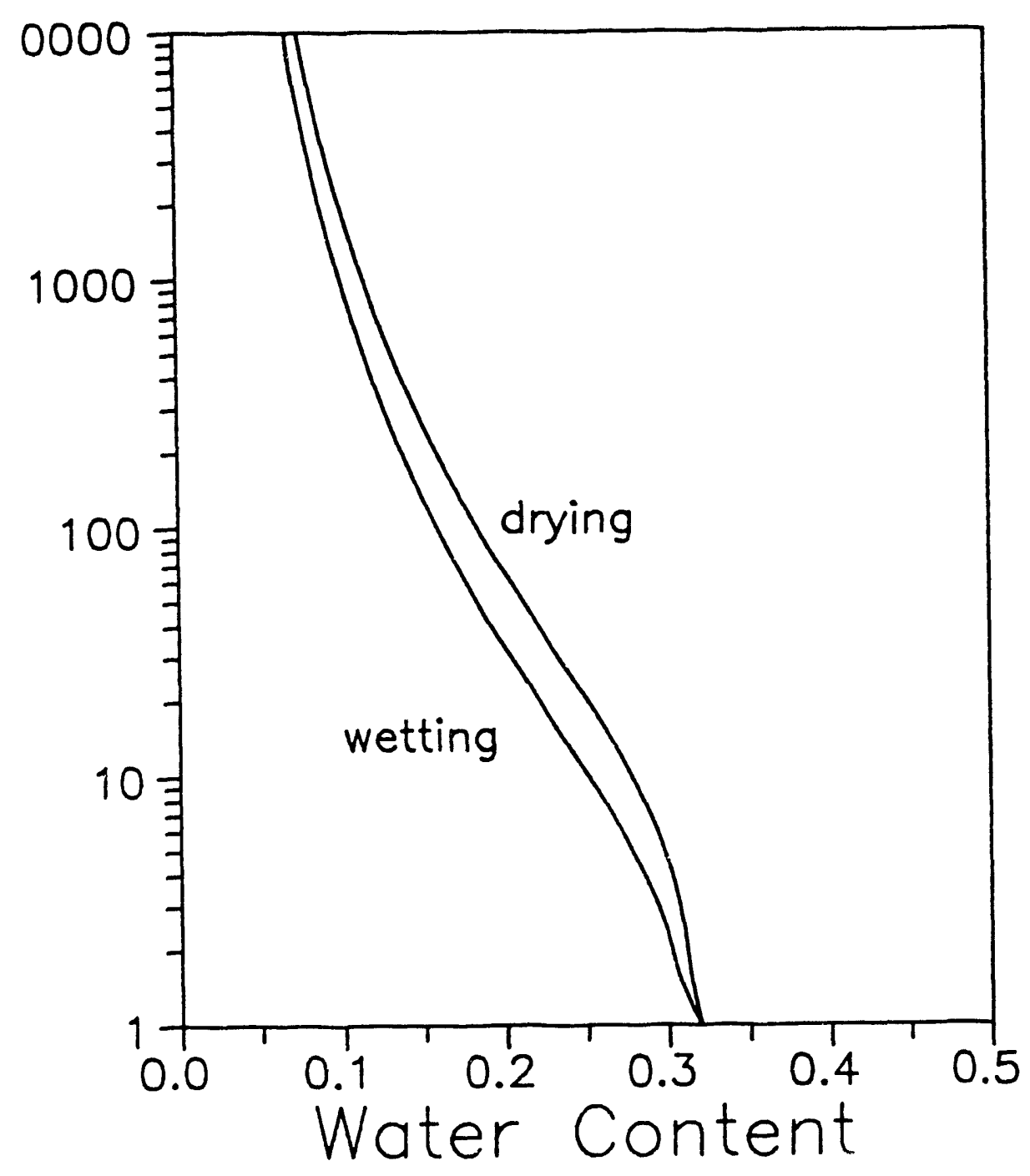

Figure 8. Wetting and drying moisture characteristic curves used in the hysteretic flow simulation (Scenario IIc). 


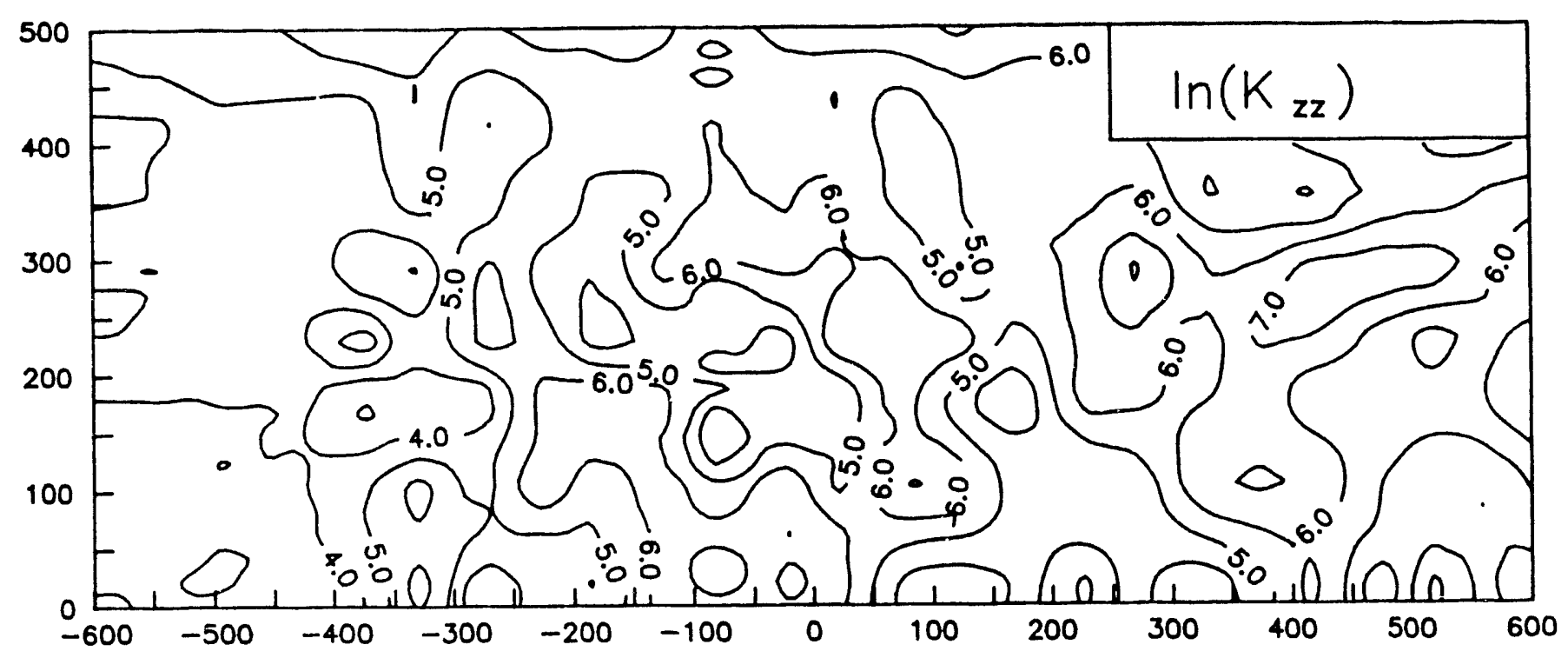

Figure 9. Spatial variation of $\ln \mathrm{K}(\mathrm{cm} / \mathrm{d})$ for Scenario III. 
concentration monitoring data however showed noticeable divergence of the tritium and bromide plumes. The probable cause is the influence of anion exclusion on bromide migration (Wierenga et al., 1990). For this reason, the transport simulations of a generic, non-reactive solute were compared against the tritium field data only. Radioactive decay of tritium during the simulation period was ignored. The error due to ignoring tritium decay is on the order of $4 \%$ at the endof the 277 day simulation period (Wierenga et al., 1990). The only additional parameters necessary for the transport simulations were therefore the longitudinal and transverse dispersivities, $\alpha_{\mathrm{L}}$ and $\alpha_{\mathrm{T}}$, and the molecular diffusion coefficient, $\mathrm{D}^{\circ}$. In contrast to the detailed data on soil hydraulic properties, there is little data available on transport parameters for the Las Cruces trench. The following dispersion parameters were assumed for all simulations: $\alpha_{\mathrm{L}}=\alpha_{\mathrm{L}}=5.0 \mathrm{~cm} ; \mathrm{D}^{\circ}=$ $1 \mathrm{~cm}^{2} / \mathrm{d}$ (Table 1). These values are the same as used by Wierenga et al. (1990) in their flow and transport simulations and thus facilitate comparison of their transport predictions with the VAM2D results. Since the value assigned to the dispersivity in the advection-dispersion transport model represents the uncertainty about local variations in pore water velocity, an argument can be made that larger dispersivity values should be used in the simulation with averaged hydraulic parameters (I, II), and a smaller dispersivity in the case of Scenario III. In the latter case the variation in hydraulic properties was described in detail, and thus a more accurate representation of local pore water velocity fluctuations would presumably be obtained in this case. The effect of varying dispersivity values was however not explored in this study.

\subsection{MODEL EVALUATION CRITERIA}

To evaluate flow and transport results for the different simulation scenarios, predicted water content and solute distributions at $t=277$ days after the start of irrigation were compared against field results. To accommodate the fact that different initial water content distributions were used, we follow the convention of Wierenga et al. (1990) to evaluate the flow simulations on the basis of water content changes, $\theta$, rather than actual water contents, where

$$
\theta(x, z, t)=\theta(x, z, t)-\theta(x, z, t=0)
$$

Two dimensional $(x-z)$ field water content distributions were available from the three neutron probe rows installed parallel to the trench. If the actual flow regime in the field would have been two-dimensional, then the total water content increase measured along each individual neutron probe row should have been the same with differences only in the spatial distribution due to local soil heterogeneity. In reality, there were significant differences between the three sets of neutron probe data (Figure 10), indicating that appreciable three-dimensional flow occurred in the field. The observed water contents for the three sets of neutron probe tubes shown in Figure 10 show that the water content increase at $y=2 \mathrm{~m}$ distance from the trench is substantially less than expected assuming uniform application and true 2-D flow. Measurements at the center $(y=6 \mathrm{~m})$ row of neutron probe tubes indicate an excess of moisture at this distance, while a mass-balance of the water contents at $y=10 \mathrm{~m}$ indicates that at this distance the water content increase agrees fairly well with the amount of applied water under 2-D flow conditions. Deviations from strictly 2-D flow were probably caused by the spatial 

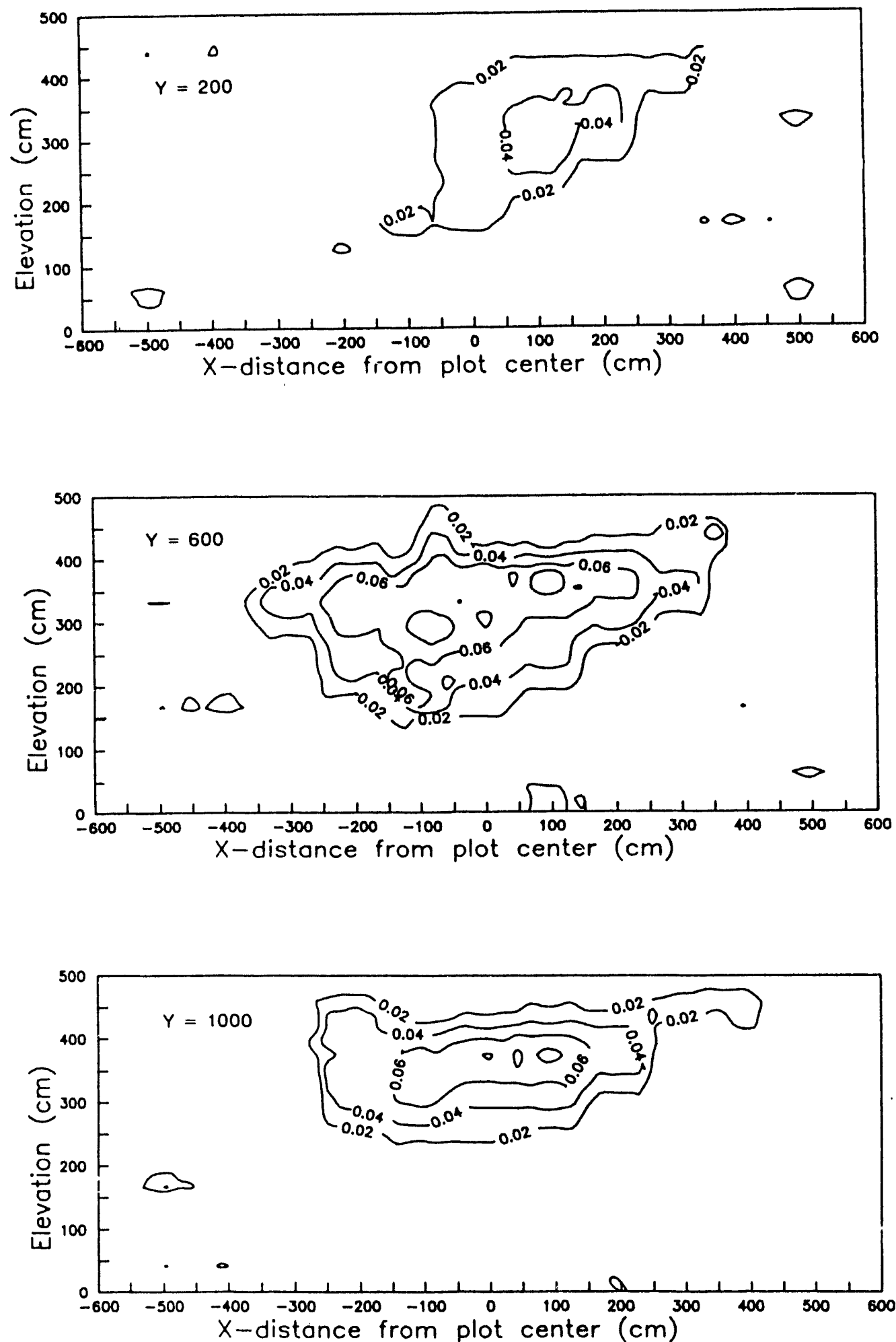

Figure 10. Water content increases at 276 days measured in three neutron probe rows; $y$-value indicates distance from trench face $(\mathrm{cm})$. 
variation of soil hydraulic properties and initial water content distribution. Non-uniformity in the water application during the irrigation period is expected to have contributed only little to the observed soil moisture distribution. To minimize the influence of three-dimensional flow in comparisons with the two-dimensional simulations, the water content data from the three neutron probe rows were averaged in the third dimension, i.e., along the $y$-axis. Tritium concentration data for transport modeling evaluation were available from a single twodimensional grid of solution samplers, all of which were installed at a distance of $50 \mathrm{~cm}$ away from the trench face. The significance of lateral ( $y$-direction) solute migration on measured concentration data could thus not be evaluated directly.

In addition to visual comparisons of contour plots of field data and simulated results, spatial moments of water content and solute distributions were computed and compared. This provides a quantitative measure of the goodness of model predictions. The ij-th spatial moment of the water content change, $\mathrm{M}_{\mathrm{ij}}^{\Theta}(\mathrm{t})$, is defined as (Freyberg, 1986)

$$
M_{i j}^{\Theta}(t)=\int_{-\infty}^{\infty} \int_{-\infty}^{\infty} \Theta(x, z, t) x^{i} z^{j} d x d z
$$

Analogously, the $i j-t h$ moment of the solute mass distribution, $\mathbf{M}_{\mathrm{ij}}^{\mathrm{c}}(\mathrm{t})$ is defined as

$$
M_{i j}^{c}(t)=\int_{-\infty}^{\infty} \int_{-\infty}^{\infty} \theta(x, z, t) c(x, z, t) x^{i} z^{i} d x d z
$$

Of most interest are the lower-order moments, i.e., the zero, first and second moments $(i+j=$ 0,1 or 2 , respectively). The zero-th moment is equal to the total mass present in the system. The first moments, normalized by total mass, define the location of the center of mass (Freyberg, 1986), in the $\mathrm{x}$ - and $\mathrm{z}$ - directions

$$
\begin{aligned}
& x_{c}=M_{10} / M_{00} \\
& z_{c}=M_{01} / M_{00}
\end{aligned}
$$

The second normalized moment determines a spatial covariance tensor:

$$
\sigma=\left[\begin{array}{cc}
\sigma_{x x} & \sigma_{x z} \\
\sigma_{z x} & \sigma_{z z}
\end{array}\right]
$$

with 


$$
\sigma_{x x}=M_{20} / M_{00}-x_{c}^{2} ; \sigma_{z z}=M_{02} / M_{00}-z_{c}^{2} ; \sigma_{x z}=\sigma_{z x}=M_{11} / M_{00}-x_{c} z_{c}
$$

The components of this covariance tensor provide a measure of the spread of the water and solute plumes about their center of mass. Since the zero-th moment (mass added) was the same in all modeling scenarios considered we used first and second moments to evaluate the model simulations. 


\section{RESULTS AND DISCUSSION}

In this section, modeling results are presented and evaluated against field observations. Flow modeling is discussed first, followed by transport modeling.

\subsection{FLOW MODELING}

Results of the Scenario I, IIa and III simulations, as well as field results are presented in Figure 11. In all cases, contour plots of water content increases at the end of the simulation $(t=277$ days) are shown. The field results correspond to measurements at $t=276$ days. During the redistribution phase moisture movement was very slow, so that the 1 day time difference between simulation results and field data has negligible influence on the comparison. The field data represent averages from the three rows of neutron probes. By averaging the field data in the y-direction, most of the effects of three-dimensional flow could be removed. It should be noted however that the amount of water present in the averaged field results (Figure 10), is approximately $15 \%$ higher than it should be based on strictly 2-D flow. As a consequence, the field results appear somewhat "wetter" than any of the simulations. The field results show a distinct horizontal spreading of the moisture plume and rather irregular water content contours. The degree of horizontal spreading is not an artifact of the averaging applied to the field data, but was also exhibited by water content distributions measured along individual neutron probe rows (see Figure 10). The predicted moisture plumes for the four different modeling scenarios reproduced the observed results to varying degrees. The uniform soil Scenarios I and IIa predicted moisture plumes which are smooth and symmetric about the $\mathrm{x}=0$ axis. Differences between Scenarios I and IIa are primarily due to differences in the assigned soil moisture retention curves (Figure 12). The curve obtained from the literature for Scenario I is a typical curve for a light textured (sandy loam) soil, with a rather abrupt decrease in water content at pressure heads below a few tens of centimeters. In contrast, the curve for Scenario II, which is a composite of individual soil core measurements shows a much more gradual change of water content with pressure head. As a result, Scenario I over-predicted the downward movement of water, while the applied irrigation was retained as shallower depths in Scenario IIa, which agrees better with observed results. The horizontal spread of the plume between 350 and $400 \mathrm{~cm}$ elevation in Scenario IIa reflects the influence of depth-varying initial water contents. Scenario III resulted in quite good visual agreement with field observations. Although this heterogeneous soil scenario did not necessarily result in accurate point-wise prediction of field results, it did reproduce the observed non-symmetric plume shape and irregular water content contours. Upon closer inspection it can be seen that the horizontal spreading in the simulation occurs mostly around the edges of the water plume. The contours in the wetter center of the plume indicate more dominantly downward flow. In contrast, the field data show distinct horizontal spreading at all contour levels. This was exhibited both by the average result as well as by data from the individual neutron probe rows.

Comparison of Scenario IIa and III results indicates that explicit consideration of soil variability, as opposed to using averaged soil properties, results in better agreement with field 

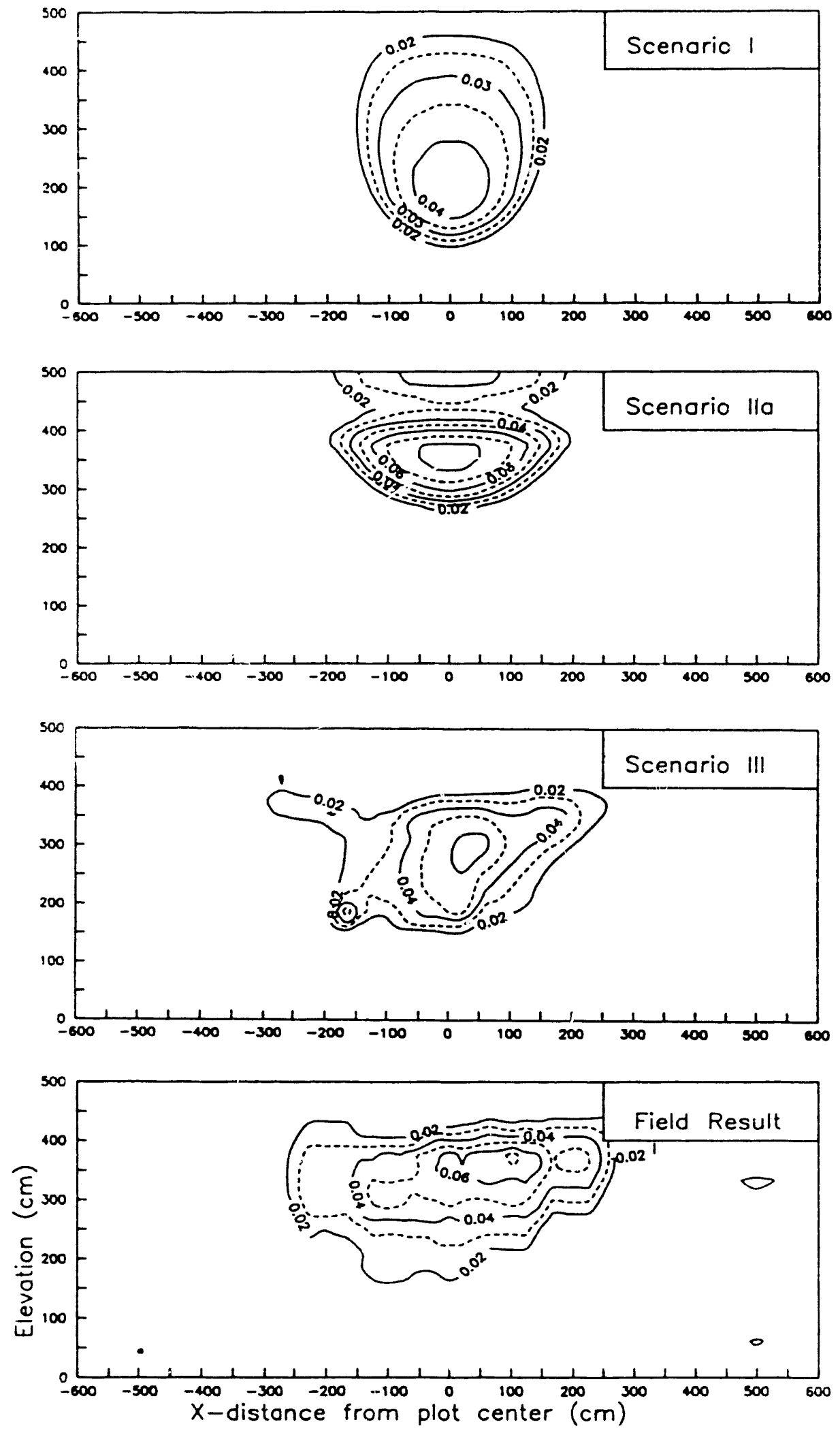

Figure 11. Comparison of predicted water plumes at $t=277$ days for Scenarios I, IIa, and III with field results. 


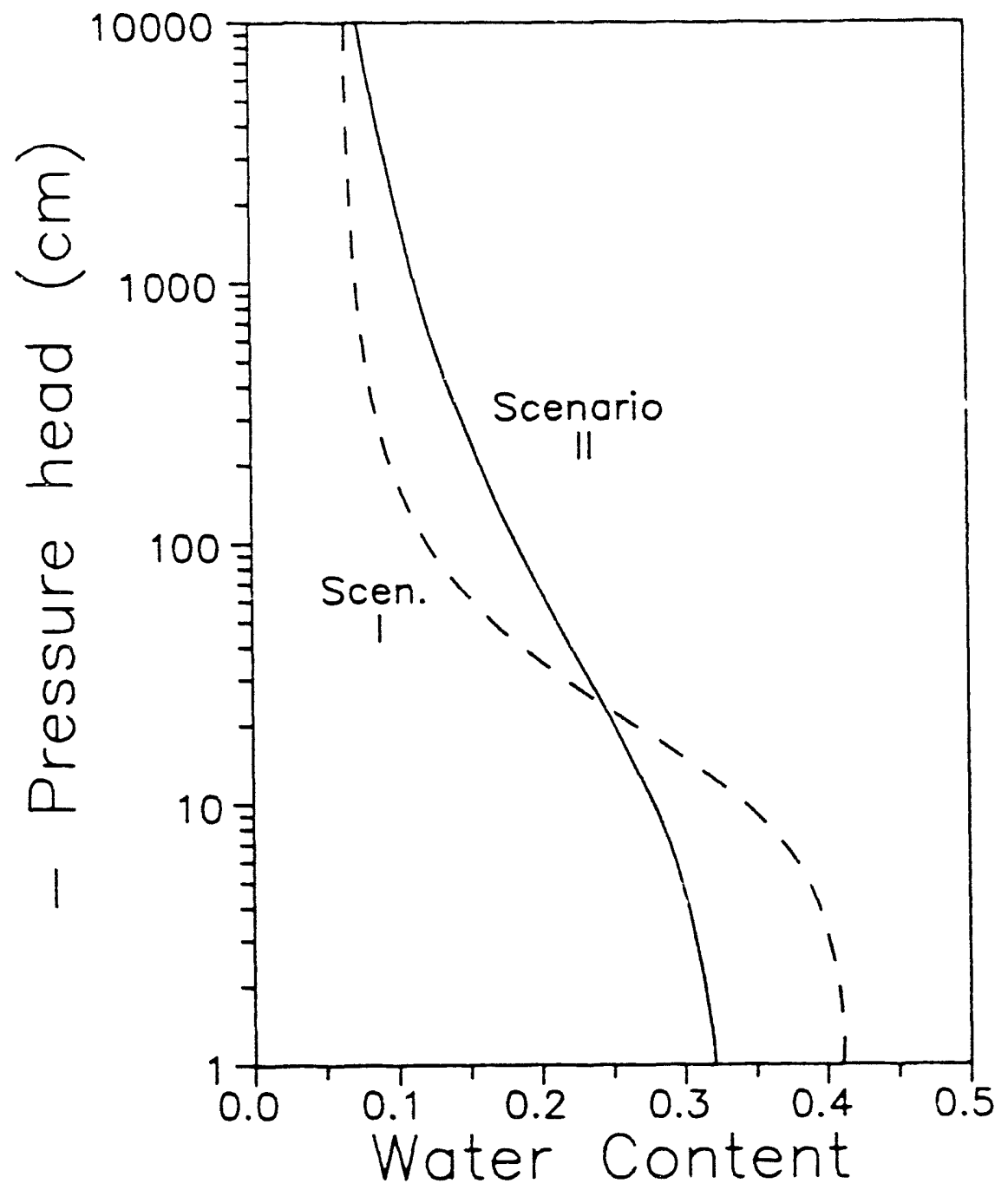

Figure 12. Soil water retention curves used in the Scenario I and II flow modeling. 
observations. One of the features of both the field data and Scenario III modeling results is the distinct horizontal spreading of the moisture plume. The horizontal spreading was not reproduced at all in the uniform soil scenario I. Some spreading can be seen in the result for scenario IIa. The spreading in the plume reflects the vertically non-uniform initial condition used in this model scenario. The degree of horizontal spreading was however much less than observed in the field. The observed spreading resembles the effect of anisotropy in soil hydraulic properties. It is unknown to what extent $t$ ' e actual soil at the trench site is anisotropic (see below). All soil cores were taken vertically downward, and parameters measured from these cores, especially saturated conductivity, thus represent vertical values. In the case of the Scenario III simulation, the apparent anisotropy was strictly a result of the local variability of soil properties, since isotropy was assumed in the modeling.

In order to evaluate the effect of anisotropy on modeling results, a variant of Scenario IIa was included in the modeling as Scenario IIb. The purpose of Scenario IIb was to see to what extent field results could be reproduced by assuming anisotropy in an otherwise uniform soil. In Scenario IIb, the average value of hydraulic conductivity, i.e., $\left\langle\mathrm{K}_{\mathrm{zz}}\right\rangle$, was recalculated assuming a lognormal distribution of conductivity values. The horizontal conductivity was then assigned a value of twice the mean vertical conductivity to produce the desired anisotropic effect. Flow results for Scenario IIb are compared with field results and with results from Scenario III in Figure 13. It can be seen that a 2:1 anisotropy ratio induced horizontal spreading similar to that observed in the field. The vertical spread of the Scenario IIb plume however, was less than either that observed in the field or predicted by Scenario III.

The mean conductivity in Scenario IIb was calculated from a lognormal distribution, rather than as a geometric mean as in Scenario IIa. The former value is about 2.5 times greater than the geometric mean value. The main motivation for using the larger value in Scenario IIb was to obtain sufficient vertical plume movement in the simulation. Using the geometric mean value in the anisotropic simulation resulted in a much too low predicted plume depth after 277 days. Lognormal probability distributions of saturated hydraulic conductivity have quite often been observed in hydrologic studies (e.g., Peck, 1983; Sudicky, 1986) and this lends some justification to the procedure for calculating $\langle K\rangle$. Interestingly though, the actual probability distribution of saturated conductivity of core samples from the Las Cruces trench does not appear to follow a lognormal distribution. The experimental data could not be described well with either a normal, lognormal or exponential distribution (Goodrich and Davis, 1989). Comparison of soil core and in-site conductivity measurements does suggest higher $\mathrm{K}$-values in the horizontal than vertical direction. However, conclusive data does not exist on the actual existence and magnitude of hydraulic conductivity anisotropy at the site. The a priori justification for selecting the hydraulic conductivity values in Scenario IIb is thus rather slim. Nevertheless, the simulation demonstrates that the infiltration of water in heterogeneous soil could be reproduced reasonably well by assuming anisotropy in an otherwise uniform soil.

Other factors that could influence the degree of horizontal spreading of the moisture plume are the presence of layers with different hydraulic properties and the influence of hysteresis in the soil moisture characteristic. Quite possibly, the observed result may reflect the 

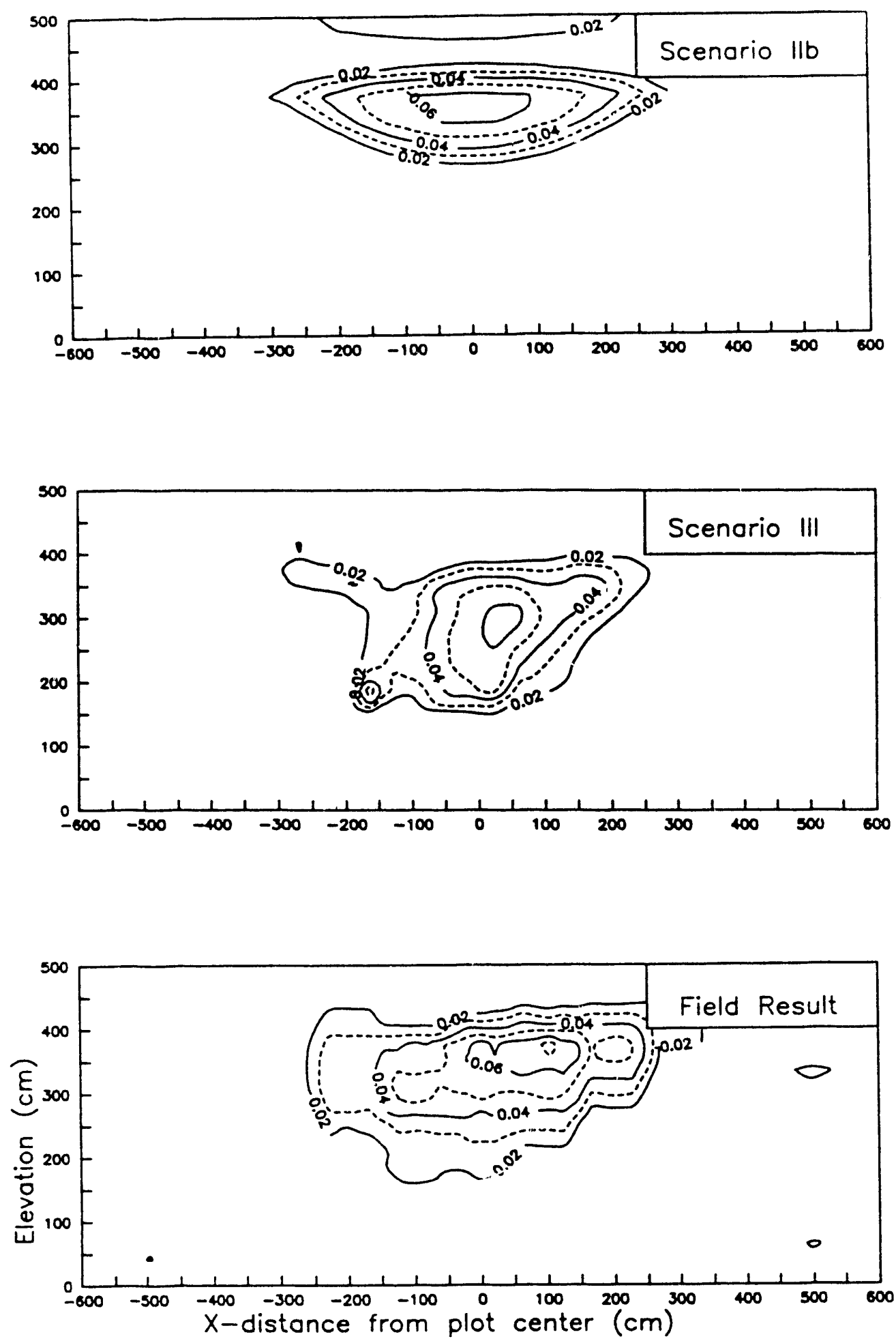

Figure 13. Comparison of predicted water plumes at $t=277$ days for Scenarios IIb and III with field results. 
influence of a combination of factors. Nine different soil layers have been identified in the upper $6 \mathrm{~m}$ of the soil profile at the site (Wierenga et al., 1989). The different layers have beendistinguished based on visual criteria which may or may not correlate with differences in hydraulic properties. Average values of the van Genuchten parameters for each of the morphological soil layers have been calculated also (Wierenga et al., 1989). These values indeed show differences between layers. A statistical analysis of saturated hydraulic conductivity values has been conducted by Sandia National Laboratories (Goodrich, 1990). This analysis suggested that three, rather than nine, layers with significantly different saturated hydraulic conductivity exist. The analysis did not consider hydraulic parameters other than the saturated conductivity. Unsaturated flow behavior though, will depend on interactions between the entire set of parameters defining the hydraulic constitutive relations. Since this is a complex interrelationship, it is not straight-forward to relate flow responses to differences in individual model parameters. Collin et al. (1990) have direstly evaluated the impact of vertical soil variations on unsaturated flow modeling by simulating the trench experiment using a layered model with layer boundaries and properties taken from Wierenga et al. (1989). Their layered model thus falls in between the Scenarios IIa and III considered here. It was found that the layered model resulted in better agreement with experimental data especially in terms of lateral spreading than did a uniform soil model. This suggests that the presence of hydraulically contrasting, although individually uniform and isotropic soil layers may have contributed to the apparent anisotropic flow behavior observed in the field.

Another possible explanation for the observed flow behavior is that the lateral spreading reflects the influence of hysteresis in the soil moisture retention characteristic. The expected effect of hysteresis on moisture movement would be to enhance water movement during the wetting phase and retard movement during the redistribution phase. The moisture retention characteristic of porous media is well known to be hysteretic, although the effect on unsaturated flow, particularly in heterogeneous and imperfectly known field soils is less clear. This is a result of both a paucity of data and a lack of modeling tools that have capability to account for hysteresis. VAM2D is one of the few multi-dimensional computer codes that have this capability. In the absence of data on capillary hysteresis, a number of additional assumptions were made for the hysteretic flow simulation (Scenario IIc). The experimental moisture retention data for the trench site all represent primary drainage curves. As a first approximation, soil wetting and drying moisture characteristics can often be described with the same set of van Genuchten parameters, with the value of parameter $\alpha$ for the wetting curve set to twice the value of the drying curve (Kool and Parker, 1987). To verify the possible significance of hysteresis, a variant of the scenario IIa incorporating hysteresis with $\alpha^{\mathrm{w}}=2 \alpha^{\mathrm{d}}$ (See Figure 8) was simulated.

In a hysteretic simulation $i \hat{i}$ must also be specified whether the initial condition corresponds to a wetting or drying condition. The upper meter of the soil at the field site was probably in a wetting condition as a result of rainfall in the weeks prior to covering the soil. The initial condition in the lower part of the soil is uncertain. However, since this part of the profile was quite dry, assuming either wetting or drying conditions leads to the same result since the wetting and drying retention curves converge at the prevailing low pressure heads. For 
simplicity therefore, the entire profile was assigned an initial wetting condition in the simulations. Other than allowing for hysteresis, this simulation was identical to Scenario IIa.

Results for the hysteretic case are depicted in Figure 14. To facilitate comparison, the original simulation results for Scenario IIa are shown also. It can be seen that accounting for hysteresis does indeed lead to different flow predictions. However, the effect of hysteresis is to reduce, rather than enhance lateral spreading. The results presented in Figure 14 show that in the hysteretic simulation the vertical plume penetration depth after 277 days is greater, while the lateral plume extent is less, than the original simulation of Scenario IIa. The reason for this behavior is that the field experiment represents a wetting event, and water movement is determined primarily by the wetting branch of the moisture retention characteristic. The shape of the wetting $\theta(\psi)$ curve in the hysteretic simulation is similar to the $\theta(\psi)$ curve used in modeling scenario I, and the moisture distribution simulated by scenario IIc approximates the result of scenario I. This results in greater downward movement and less lateral spreading of the moisture plume at the end of the 277 day period compared to the simulation based on the drying branch of the moisture retention characteristic used in the original Case IIa scenario. The effect of hysteresis in the simulation is completely the opposite of the observed field behavior. This suggests that capillary hysteresis either does not have a significant effect on water movement, or if hysteresis is present, its influence is masked by other, opposing processes.

First and second values of the plume moments (Table 2) confirm conclusions from the visual plume comparisons. Since the effect of hysteresis in the field experiment was apparently not significant, the Scenario IIc was omitted from the moment analysis. All modeling scenarios predict essentially vertical downward movement of the plume center-of-mass, i.e., a negligible value for $\mathbf{M}_{10}$. In contrast, the field results show a distinct sideways movement of the moisture plume. The extent of downward movement of the plume center-of-mass $\left(M_{01}\right)$ is overpredicted in Scenarios I and III, but underpredicted in Scenarios Ila and IIb. The amount of under- and overprediction, respectively, in Scenarios IIb and III, is about the saine. The plume second moments clearly illustrate the horizontal spreading of the moisture plume observed in the field. The spread of the plume is approximated most closely in Scenario III, while it can be seen again that Scenario I results in a poor prediction of the plume shape; this scenario predicts a vertically, rather than horizontally, elongated plume. 

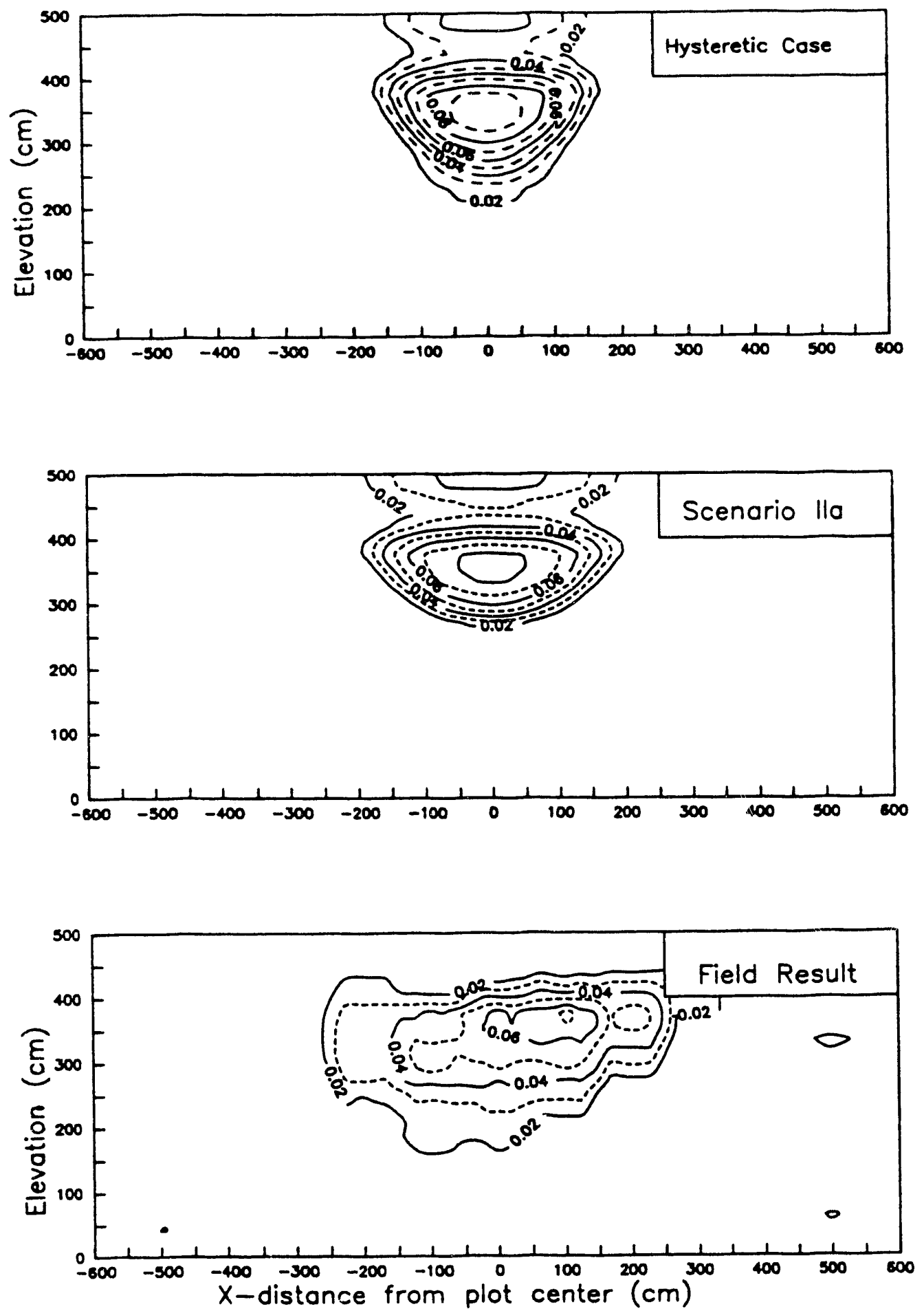

Figure 14. Predicted moisture plume at $t=277$ days for hysteretic case (Scenario IIc) compared with the non-hysteretic simulation (Scenario IIa) and field result. 
Table 2. Normalized Spatial Moments of Simulated and Observed Water Plumes

\begin{tabular}{lrrrrrr}
\hline \multicolumn{1}{l}{ Moments } & I & \multicolumn{2}{c}{$\begin{array}{l}\text { Scenario } \\
\text { Ila }\end{array}$} & IIb & III & Field \\
\hline $\mathrm{M}_{10} \quad(\mathrm{~cm})$ & -0.4 & 0.3 & 0.4 & 7.3 & 60.5 \\
$\left.\mathrm{M}_{01}{ }^{1}\right)$ & $(\mathrm{cm})$ & 287.2 & 376.4 & 366.3 & 274.4 & 318.9 \\
$\mathrm{M}_{20}$ & $\left(\mathrm{~cm}^{2}\right)$ & 8272.4 & 28984.9 & 43978.8 & 52826.4 & 60109.5 \\
$\mathrm{M}_{02}$ & $\left(\mathrm{~cm}^{2}\right)$ & 11086.4 & 6650.0 & 6676.3 & 7112.7 & 8859.1 \\
\hline
\end{tabular}

1) Reflects z-coordinate convention used in modeling, i.e., $z$ is positive upwards with soil surface at $z=500 \mathrm{~cm}$.

In summarizing the results of the VAM2D flow simulations, it is clear that the most accurate result is obtained with the Scenario III simulation which accounts on closely as possible for local scale soil heterogeneity. A key feature of the observed field behavior, namely the lateral spreading of the moisture plume, can be reproduced by allowing for anisotropy in the saturated conductivity. Since no data on directional hydraulic conductivities are available, it is not possible to determine how significant local anisotropy actually is, or whether the apparent anistropy results solely from local soil heterogeneities. The good results obtained with simulation Scenario III suggest that the latter explanation may be the most appropriate.

The notion that the effect of local heterogeneities may manifest itself as an apparent large scale anisotropy is supported theoretically by stochastic unsaturated flow models developed by L.W. Gelhar and co-workers at MIT. One conclusion from this work is that the anisotropy of effective conductivity in unsaturated media is dependent on the mean pressure head. Yeh et al. (1985) have presented the following expression for the apparent anisotropy of hydraulic conductivity under unit gradient flow with soil layering parallel to the $\mathrm{x}$-axis:

$$
\frac{\hat{k}_{x x}}{\hat{k}_{z z}}=\exp \left[\frac{\sigma_{f}^{2}+\sigma_{a}^{2} \psi^{2}}{1+A \lambda}\right]
$$

where ^ denotes effective value and $\mathrm{A}, \lambda, \sigma_{\mathrm{f}}$ and $\sigma_{\mathrm{a}}$ are soil dependent parameters related to the mean, isotropic, hydraulic conductivity and its spatial covariance function. Specifically, $\sigma_{\mathrm{f}}^{2}$ is the variance of the $\log$ saturated conductivity $\left(\ln \left[K_{s}\right]\right), A=\int \ln k_{\mathrm{T}} / d \psi$ is the mean value of the slope of the $\ln k_{\mathrm{T}}(\psi)$ relation, $\sigma_{\mathrm{a}}^{2}$ is the variance of $\mathrm{A}$, and $\lambda$ is the correlation length in the 
direction perpendicular to the soil layering. The stochastic flow theory as represented by (20) suggests that soils which behave as uniform media under relatively wet conditions will exhibit increasing effects of heterogeneity as they become drier. Inspection of (20) shows that it predicts that the apparent anisotropy will vary as the exponential of pressure head squared. Equation (20) can be incorporated in a numerical flow solution in a straight-forward manner, and provided the necessary statistical parameters are known, it allows evaluation of this aspect of the stochastic unsaturated flow theory in an otherwise deterministic flow simulation. McCord et al. (1988) have used (20) in numerical simulations of subsurface water and solute movement along a hillslope and have obtained good qualitative agreement between simulations and observed data. The implementation of (20) in VAM2D is discussed by Huyakorn et al. (1989), following in part suggestions of Polmann et al. (1988). Polmann et al. (1988) also provide values of the parameters in (20) for the Las Cruces site, and which are summarized in Table 3. First of all, it may be noted that (20) provides an expression for the anisotropy ratio, but not for the actual, directional relative permeabilities themselves. In order to obtain the desired, monotonically decreasing $\hat{\mathrm{k}}_{\mathrm{xx}}(\psi)$ and $\hat{\mathrm{k}}_{\mathrm{zz}}(\psi)$ relations, an empirical logarithmic interpolation procedure was developed which ensures that both $\hat{k}_{x x}$ and $\hat{k}_{z z}$ decrease with decreasing pressure head while maintaining the correct anisotropy ratio for any $\psi$ value.

Table 3. Statistical parameters describing the anisotropic $\mathrm{k}_{\mathrm{r}}(\psi)$ relation. Parameter values from Polmann et al. (1988).

Parameter Value
A $\left(\mathrm{cm}^{-1}\right)$
0.117
$\sigma_{\mathrm{a}}^{2}\left(\mathrm{~cm}^{-2}\right)$
0.0007
$\sigma_{\mathrm{f}}^{2}$
0.36
$\lambda(\mathrm{cm})$
25.0

In implementing the algorithm in the VAM2D code, it was found that the predicted anisotropy ratio is quite sensitive to the chosen values of $\mathrm{A}$ and $\sigma_{\mathrm{a}}^{2}$. The stochastic theory is based on an exponential relationship between relative permeability and pressure head

$$
k_{r}=e^{\alpha \psi}
$$

where 


$$
\alpha=A+a
$$

and $\mathrm{a}$ is the local variation of $\mathrm{A}$, with zero mean and variance $\sigma_{\mathrm{a}}^{2}$. In the original theory, $\mathrm{A}$ is constant, independent of $\psi$. When a different relative permeability relation, such as the van Genuchten relation (3) is employed, $A$ is no longer a constant but becomes a function of $\psi$. With the van Genuchten $k_{T}(\psi)$ relation, the corresponding value of $A$ will decrease as the pressure head $\psi$ becomes more negative. For instance, using the van Genuchten $k_{\tau}(\psi)$ relation for the Las Cruces trench soil, with parameters from the averaged $\theta(\psi)$ data (Scenario II), the value of the slope of $\ln k_{1}$ decreases from $A=0.57$ near saturation to $A=10^{-3}$ at $\psi=-2500$ $\mathrm{cm}$. Using a variable $\mathrm{A}$ therefore tends to considerably magnify the predicted degree of anisotropy. Recognizing this, Polmann et al. (1988) suggested that it would be reasonable to assume a constant coefficient of variation for $A$, i.e., a fixed ratio of $\sigma_{a} / A$. However, in this case $\sigma_{\mathrm{a}}^{2}$ will vary as $\mathrm{A}$ squared which in turn will tend to reduce the predicted anisotropy. To illustrate these different effects, the predicted anisotropy ratio for the Las Cruces trench soil is plotted in Figure 15 using (20) with different ways of calculating $A$ and $\sigma_{\mathrm{a}}^{2}$. When these parameters are fixed at their nominal value (Table 3 ), the predicted anisotropy ratio increases quickly with decreasing pressure head, up to 7 orders of magnitude at $\psi=300 \mathrm{~cm}$. When A is treated as a variable and computed from the van Genuchten $\mathrm{k}_{\mathrm{r}}(\psi)$ relation with constant $\sigma_{\mathrm{a}}^{2}=$ 0.0007 , the computed anisotropy ratio becomes even more extreme. On the other hand, when $\sigma_{\mathrm{a}}^{2}$ and $\mathrm{A}$ are both treated as variables with a fixed ratio $\sigma_{\mathrm{a}} / \mathrm{A}$, the anisotropy is effectively suppressed. In this latter case, the predicted anisotropy remains less than 1.5 over the entire range of pressure heads. The extreme sensitivity of predicted anisotropy to different, plausible ways of evaluating model parameters is clearly a problematic aspect of incorporating the algorithm into a general purpose simulator like VAM2D. This aspect of the stochastic theory is not explored further. Rather, in subsequent analyses, the parameters $A$ and $\sigma_{\mathrm{a}}$ were both treated as constants with values as given in Table 3, i.e., the modeled anisotropy ratio for the Las Cruces trench site is represented by the solid curve in Figure 15. The applicability of (20) for the trench experiment was evaluated through additional flow simulations. The Scenario IIa average hydraulic parameters were used together with (20). The result of this simulation is shown in Figure 16. As in the previous figures, the results are shown in terms of the increase in water contents after 277 days. The simulation predicts a large degree of horizontal spreading of the applied irrigation water and relatively little vertical penetration of the plume. Compared to the field result, the apparent anisotropy is significantly overpredicted by the model. The computed anisotropy ratio in the simulation is around 7:1 or $8: 1$ in the center of the plume and reaches much higher values at the drier fringes of the plume. As shown in the simulation of scenario IIb, the apparent anisotropy ratio in the field result is approximately $2: 1$. Estimating the effective anisotropy factor from the stochastic flow theory via (20) results in a poor prediction of the field experiment. As mentioned, McCord et al., (1988) have reported good qualitative agreement between field observations of solute transport in unsaturated soil and simulations employing (20). A possible explanation for the poor results obtained here, compared to the findings of $\mathrm{McC}$ Cord et al., is the drier conditions of the trench experiment. It may well be that while the assumptions and simplifications embodied in (20) hold in relatively wet soils, they failed under the much drier conditions of the second Las Cruces Trench experiment. 


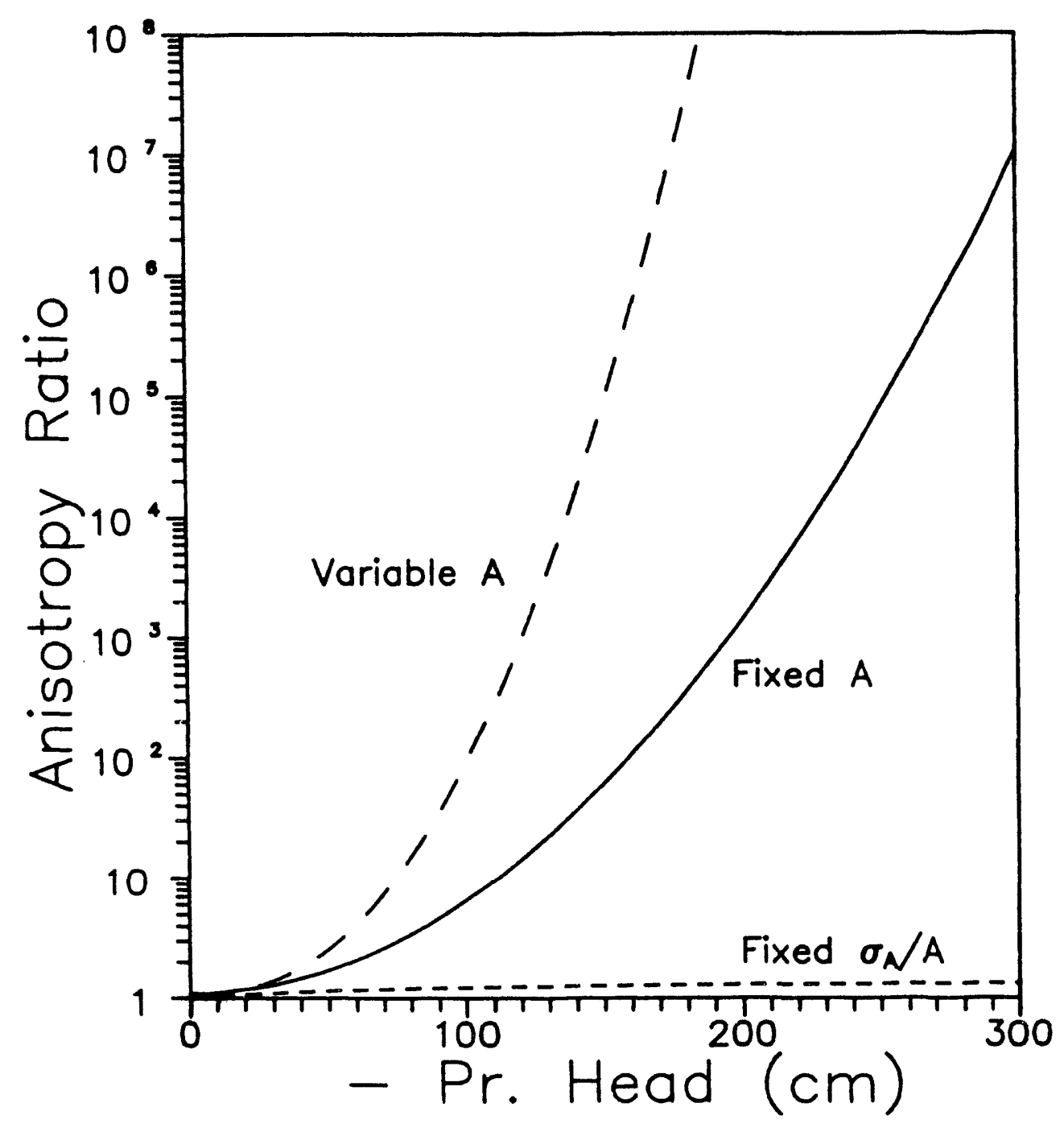

Figure 15. Anisotropy ratio as a function of pressure head, $\psi$, predicted from (20) with parameters for the Las Cruces trench site from Polmann et al. (1988) and different ways of evaluating $\mathrm{A}$ and $\sigma_{\mathrm{a}}$. 


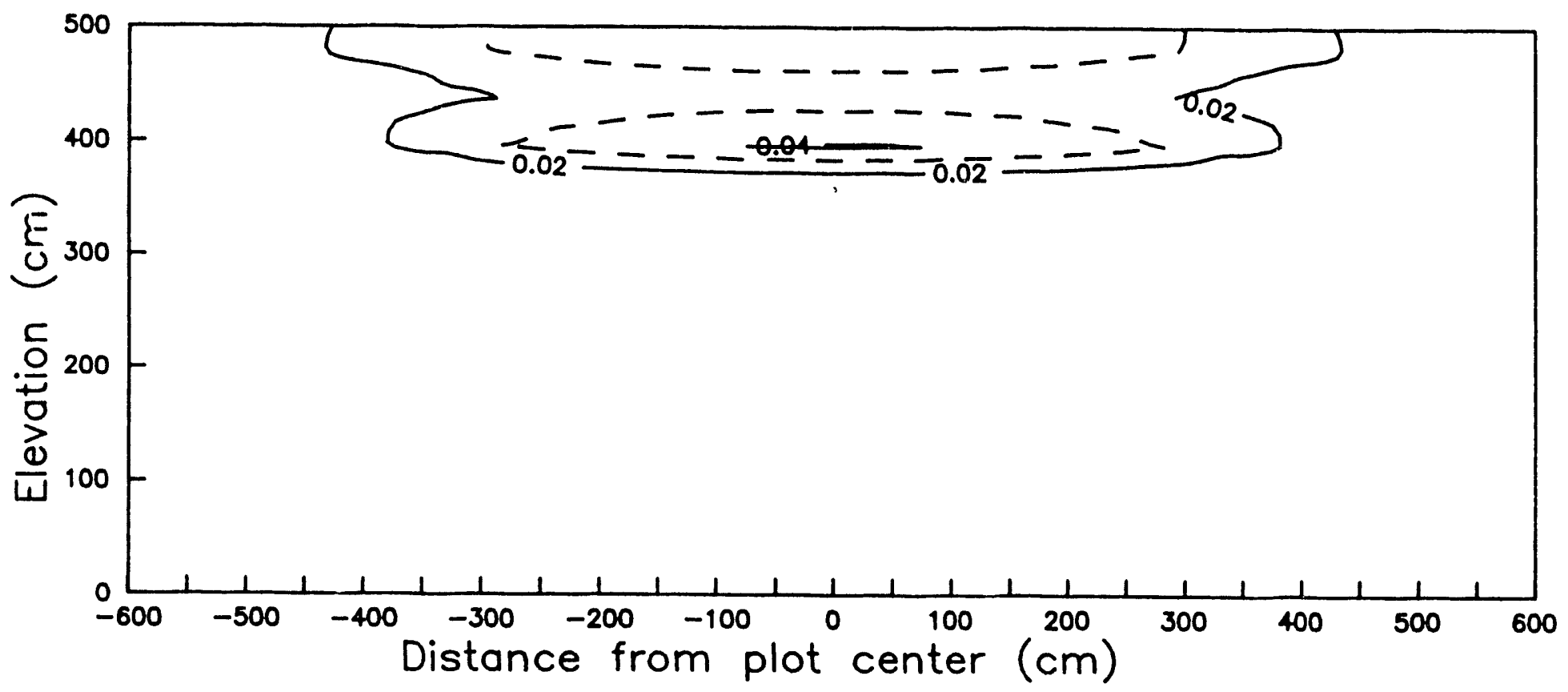

Figure 16. Moisture piume at $\mathrm{t}=277$ predicted using pressure head dependent anisotropy of the relative permeability. 


\subsection{SOLUTE TRANSPORT MODELING}

Tritium transport was simulated for the four Scenarios I, IIa, IIb, and III; Scenario IIc involving hysteresis was not considered in the transport analysis. As mentioned, the same dispersion parameters were used in all cases; differences in transport predictions thus reflect differences in flow simulations. Predicted tritium profiles were compared with data from in-situ solution samplers. As only one set of solution samplers was installed, there is no spatial averaging of concentration data as was done for the field water content data. Nor is it possible to directly evaluate the extent of three-dimensional solute migration. Predicted concentration contours for the four modeling scenarios are compared with field tritium data in Figure 17. First and second spatial moments of the solute mass distribution are given in Table 4.

Table 4. Normalized Spatial Moments of Simulated and Observed Tritium Plumes

\begin{tabular}{lrrrrrr}
\hline \multicolumn{1}{l}{ Moments } & \multicolumn{1}{c}{ I } & \multicolumn{1}{c}{ Scenario } & \multicolumn{1}{c}{ IIb } & \multicolumn{1}{c}{ III } & Field $^{\text {l) }}$ \\
\hline $\mathrm{M}_{10} \quad(\mathrm{~cm})$ & 0.0 & 0.0 & 0.0 & 17.8 & -10.9 \\
$\mathrm{M}_{01}{ }^{2}$ & $(\mathrm{~cm})$ & 361.8 & 405.5 & 418.3 & 367.5 & 423.7 \\
$\mathrm{M}_{20}$ & $\left(\mathrm{~cm}^{2}\right)$ & 4338.0 & 5578.9 & 9019.8 & 7379.1 & 9967.9 \\
$\mathrm{M}_{02}$ & $\left(\mathrm{~cm}^{2}\right)$ & 4623.0 & 2608.9 & 2165.2 & 5757.1 & 2352.9 \\
\hline
\end{tabular}

1) Computed using water content data from nearest neutron probes at $y=200 \mathrm{~cm}$.

2) Reflects $\mathrm{z}$-coordinate convention used in modeling, i.e., $\mathrm{z}$ is positive upwards with soil surface at $z=500 \mathrm{~cm}$.

In calculating the field moments, water content measurements from the neutron probe row at $y=200 \mathrm{~cm}$ (i.e., nearest the solution samples) were used in (17). As expected, the predicted tritium plume for each scenario follows the flow results shown in Figures (11) and (13). Compared to the flow results in Figures 11 and 13, it can be seen that the solute front in all cases lags behind the water front. This is due to a "snowplow" effect in the flow modeling, in which displaced antecedent water is pushed ahead of the infiltrating water and solute front. The predicted depth of tritium penetration is significantly greater for Scenarios I and III than observed in the field. The overall plume shape for Scenarios IIa and IIb shows better agreement with the field result. Upon closer inspection it is seen however, that the concentration values observed in the field are substantially larger than maximum values in any of the four simulation scenarios. The highest measured relative concentration value at $t=277$ days was about 0.45 , 

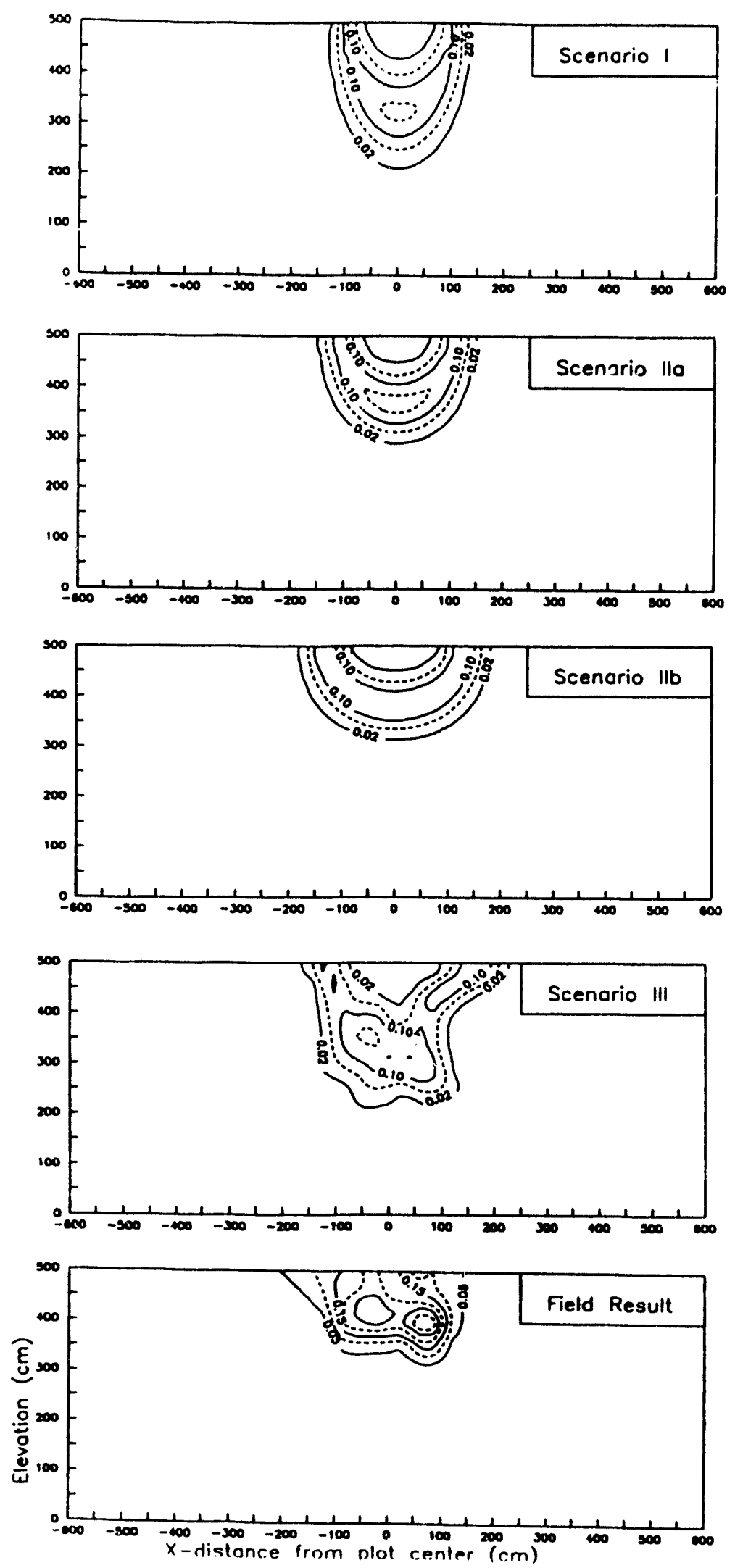

Figure 17. Predicted concentration distributions at $t=277$ days for four modeling scenarios and field results. 
while none of the predicted concentrations was higher than 0.17 . A partial reason for this discrepancy may have been overestimation of dispersivities and thus greater spreading in the modelled results. However, this cannot explain the apparently much larger solute mass measured in the field than was present in the simulation results. As mentioned, the solute mass present in the simulations was equal to the applied mass to within about $2 \%$. The recovered field solute mass cannot be directly calculated since there were no coincidental water content and concentration measurements. The high concentration measurements in the field may in fact reflect much lower actual than predicted water contents at the solution sampler locations. Water content data from the nearest neutron probe row at $y=200 \mathrm{~cm}$ from the trench show indeed much lower than average water contents (Figure 10). However, even if these water contents are combined with measured tritium concentration data, the resulting solute mass as estimated by the zero-th moment of the field tritium distribution is still approximately $80 \%$ higher than the actually applied amount based on uniform application and two-dimensional transport. Lacking more spatially detailed solute data, it can only be surmised that the high concentration values observed in the field represent a solute and/or water content distribution that is highly uneven in the y-direction. Since the discrepancy with simulated results is so large, the field observations cannot really be used to judge the individual simulations. Still, it is of some interest to note that Scenario III, which resulted in the best flow predictions, does not result in the closest transport predictions. Based on the first and second spatial moments (i.e., plume shape); Scenario IIb results in the best approximation of the field solute distribution. 


\section{CONC.LUSIONS}

The first observation in evaluating modeling results of the second trench experiment against field flow and trarisport data, is the significant influence of three-dimensional flow and transport in the field. Even though the field experiment was designed to produce twodimensional flow and transport, as much as $40 \%$ of the applied water may have moved in the lateral y-direction (INTRAVAL 1989 Progress Report). Water content data from three rows of neutron probes access tubes are available. By averaging data in the third dimension (y-direction) it was possible to minimize the 3-D flow effects and obtain a set of field results that can be used to evaluate the two-dimensional modeling results. Only one set of measured concentration data is available. Coupled with the lack of coincidental water content measurements, the available concentration data are too limited to provide a basis for rigorously evaluating the transport modeling results.

The flow simulations indicate the strong influence of soil heterogeneity on water infiltration and redistribution. In evaluating the flow simulations, we observe that in two cases, Scenario IIb and III, good agreement with field data was obtained. The use of non-site specific model calibration data in Scenario I on the other hand resulted in poor predictions. Results of the Scenario IIc suggest that hysteresis is not a significant factor in this experiment. It should be noted that no parameter adjustment was performed in Scenario III in order to match the field results. Scenario IIb on the other hand, was included as one of the modeling scenarios following evaluation of Scenario IIa. Hydraulic parameters for IIb were deliberately chosen to reproduce the observed horizontal plume spread.

We did not obtain very good agreement between observed and simulated tritium transport. The ability to quantitatively evaluate the solute transport simulations is limited due to the lack of coincident water content and concentration data and the lack of spatial resolution in the solution sampler data, especially with respect to three-dimensional solute migration. There was significant water movement in the third $(\mathrm{y}-)$ dimension and presumably the same applies to solute movement in the experiment. Based on predicted and observed tritium plume shapes, the uniform but anisotropic soil model (Scenani IIt) jielded results at least as good as the simulation based on the more complex, heterogeneo ss soil model (Scenario III). It would probably be wrong to conclude from this that the Scenario IIa represents a more accurate description of the actual field site than Scenario III. However, this fact does illustrate that when the conceptualization of important processes is incomplete (e.g., ignoring 3D migration) and/or when sufficient data for model calibration and validation are lacking, a more complex model may not provide any more reliable results than a simpler model.

These results suggest the desirability of incorporating as much information as possible about soil heterogeneity in a computational model. In the absence of detailed data, it may be possible to account for the effects of soil variability as an apparent large scale anisotropic effect. It is less clear, however, how to a priori determine the effective anisotropy or even, as illustrated in the case of calculating the effective mean saturated hydraulic conductivity, how to average variable soil properties to obtain a meaningful effective value. The finding that local 
soil heterogeneity may manifest itself as an apparent large scale anisotropy, agrees qualitatively with conclusions from stochastic flow theories developed at MIT. In this case, however, we were not successful in using the stochastic theory to predict the large scale hydraulic conductivity. This may reflect a deficiency in the underlying theory, or alternatively, the hazards of using an isolated result from this theory, i.e., Equation (20), outside of the context of the stochastic theory of which it is a part.

Finally, referring back to the first objective of this study, the simulations of the Las Cruces trench experiment have proven to be a very valuable test for the computational routines employed in VAM2D. Difficulties encountered in initial simulations have led to further improvements to the numerical schemes used in VAM2D. Simulations reported here used the various procedures discussed in Section 2.2.2 to achieve an accurate and numerical solution. Our results show that it is indeed feasible to obtain accurate and mass-conservative solutions to the variably saturated flow and transport equations, even when sharp saturation fronts are present, provided the numerical schemes are internally consistent and coefficients are evaluated in a consistent and appropriate manner. 
PART 2

APPLICATION OF THE VAM2D COMPUTER CODE FOR MODELING OF FLOW AND TRANSPORT IN FRACTURED MEDIA USING AN EQUIVALENT POROUS MEDIUM APPROACH 


\section{SUMMARY}

This study investigates the applicability of the equivalent porous medium (EPM) approach for predicting contaminant migration in fractured groundwater systems. In the absence of detailed site characterization and flow and transport validation data for a field site, a hypothetical disposal site overlying a fractured aquifer is considered. Steady state hydraulic head and transient solute concentration distributions are generated by performing detailed simulations using the FRACTRAN discrete fracture flow and transport model. Values of the effective flow and transport parameters used in the porous medium approximations are determined from knowledge of the fracture orientation, spacing and apertures, using relationships that are readily available from the literature. Expressions for effective transport parameters given in the literature pertain to one-dimensional transport scenarios, but are applied here to a two-dimensional flow and transport problem. The VAM2D computer code is used to perform the porous medium simulations. Three different transport scenarios are considered involving conservative as well as reactive contaminants. The VAM2D results are compared to the 'true' FRACTRAN results for the three scenarios. In all cases, the VAM2D results are based on flow and transport parameters computed directly from the fracture characteristics, without any additional calibration. VAM2D results are found to closely duplicate the FRACTRAN hydraulic head distribution, indicating that the porous medium approximations are quite accurate for flow modeling. In all three transport cases, agreement between the VAM2D predictions and 'true' FRACTRAN results was reasonable to good. The agreement improves when diffusional exchange between fractures and the porous matrix is relatively rapid. The results of this study suggest that for modeling problems of a practical scale, porous medium approximations may be adequate, and that provided accurate fracture characterization data is available for determining values of the effective flow and transport parameters, porous media models can be to predict flow and transport behavior. 


\section{INTRODUCTION}

In the great majority of cases where computer models are used to predict groundwater flow and/or migration of dissolved chernicals in the subsurface, the geologic medium is assumed to behave as a porous medium. This assumption is either made explicitly or implicitly via the use of Darcy's Law and the advection-dispersion equation to describe flow and transport, respectively. At the same time, it is increasingly recognized by hydrogeologists that many natural geologic media do not behave as idealized porous media, but often contains regions of preferential flow and transport. In the soils literature, the term 'macropores' (e.g. van Genuchten and Jury, 1987) is often used to describe this phenomenon. In groundwater hydrology the term 'fractures' is commonly used (e.g. Freeze and Cherry, 1979). We will adopt the latter term in this study. When an interconnected fracture network exists, the fractures often are the dominant pathways for movement of water and solutes. This is the case even when fracture apertures are quite narrow, e.g. on the order of microns, and the total volume of fractures contributes little to the total porosity of a soil or aquifer. On the other hand, it may not be realistic to only consider the fractures and ignore the matrix, especially when modeling solute transport. Diffusion of solute from fractures into the (porous) matrix may significantly reduce the effective rate of travel of an advancing solute plume (Neretnieks, 1980; Grisak and Pickens, 1980a, b). Conversely, the same process of matrix diffusion will, in cases of aquifer remediation, cause long-term release of contaminants back into an aquifer, and thus reduce the effectiveness of clean up schemes. In short, the presence of both fractures and matrix must be considered in any quantitative assessment of flow and transport in fractured media.

A number of different approaches can be used to achieve this objective. In order of decreasing complexity (and rigorousness) they are: the discrete fracture modeling approach, the double porosity approach, and the equivalent porous medium approach. The advantages and limitations of each approach have been discussed extensively in the literature (e.g., Parker and Valocchi, 1986; Pankow et al., 1986; Berkowitz et al., 1988; Khaleel, 1989). In the discrete fracture approach, individual fractures are modeled explicitly. Separate flow and transport equations are applied to fractures and matrix with linking through appropriate coupling terms. Limitations of this approach include the computer resources required to run a simulation code and the detailed information required to describe the fracture geometry. In the double porosity approach, fractures and matrix are considered as two separate but overlapping criteria, without any specific geometry. Diffusion is usually considered to be the only transport mechanism in the matrix, with exchange of solutes between matrix and fractures described as a first-order kinetic process. The rate coefficient and other parameters in the double porosity model are usually determined by inverse procedures, i.e., estimated from tracer tests (e.g., Parker and van Genuchten, 1984). Alternatively, the parameters can be approximated directly given data on fracture and matrix geometry (van Genuchten, 1985).

The double porosity approach is conceptually appealing and computationally much less demanding than the discrete fracture modeling approach. Nevertheless, the great majority of available groundwater flow and transport codes are based on porous media models and do not 
have the capability to consider any kind of fractured media explicitly. The alternative then may be to simply treat the fractured medium as an equivalent porous medium and model it using appropriate values of the (effective) porous medium transport parameters. The applicability of the equivalent porous medium (EPM) approach for describing flow and transport in fractured media has been investigated in a large number of studies (Snow, 1969; Long et al., 1982; Pankow et al., 1986; Parker and Valocchi, 1986; Berkowitz et al., 1988; Khaleel, 1989). These studies have yielded varying conclusions regarding the applicability of the EPM approach. Evaluations of the adequacy of the porous medium approximation may depend on the desired level of accuracy, whether flow or transport is considered, and on the type of fracture network considered. When regular and interconnected fracture systems are considered, the EPM approach is generally more satisfactory than in cases where random and/or sparsely connected fracture systems are considered. Endo (1984) concluded that for some fracture systems, fluid flow can be predicted using porous media assumptions, but that the same may not be valid for modeling solute transport. A general result is that the need to distinguish between fractured and porous media is very much a matter of scale. Flow and transport behavior in fractured media will approach that of porous media when large enough travel scales are considered. The determination of what constitutes a large enough travel scale to allow use of porous medium approximations for a fractured medium depends on a number of fracture characteristics and transport parameters.

In addition to dependence on fracture geometry, porous medium behavior will be reached more quickly if the conditions are such that exchange between the fractures and matrix is "rapid" relative to the rate of movement in the fractures. Factors that favor porous medium-like transport behavior thus include: low flow rate in fractures, closely spaced fractures, and rapid diffusion in the matrix. In an equivalent porous medium approximation of solute transport, the presence of fractures is accounted for in the effective dispersion tensor. The primary contribution to dispersion is now the velocity contrast between matrix and fracture, resulting in typically large dispersion coefficients. Expression for the effective transport parameters in EPM models of fractured media have been derived for a number of fracture geometries (e.g., Fried, 1975; van Genuchten and Dalton, 1986). Existing approximations pertain to relatively simple fracture geometries and one-dimensional, steady state flow, but they offer a means to determine effective EPM model transport parameters given information on fracture characteristics, such as spacing and mean aperture. Available results on the field validation of the EPM approach for describing fractured media are scarce. Pankow et al., (1986) discuss evaluation of the EPM approach for describing contaminant transport at two waste disposal sites on fractured media. At one site, the fracture spacing was small with a high degree of fracture connectivity and a relatively high matrix diffusion coefficient. Field observations at this site indicated a uniform flow field and a regular plume shape. Pankow et al., (1986) use these observations to argue that the EPM approach should be applicable at this site. At the second site, the fracture characteristics were less favorable for EPM approximations. Combined with the irregularity of an observed contaminant plume, it was argued that the EPM approach would not be valid at this site. The authors, however, did not present any simulation results to determine the actual modeling errors involved when using porous media approximations to model their two sites. 
A significant factor explaining the scarcity of field validation results of EPM models for fractured sites is probably the difficulty in obtaining accurate fracture characterization and model validation data. One alternative is to conduct numerical experiments, using computer generated fracture networks, to produce synthetic flow and transport data against which to evaluate EPM simulations. One such application has been discussed by Berkowitz et al., (1988). In their study, tracer breakthrough curves were generated, using a discrete fracture flow and transport model, for a two-dimensional, regular fracture network under different flow conditions. Since the shape of the breakthrough curves could be described quite well using the single continuum advection-dispersion model, it was concluded that, except for the region close to the source, the EPM model was sufficient for modeling solute transport. The authors suggested that in an actual field situation, it would be required to conduct tracer tests in order to estimate the effective EPM transport parameters. Berkowitz et al., (1986) did not consider the possibility of using information on the fracture characteristics to estimate transport parameters for the EPM model directly.

Our present study is concerned with evaluating the applicability of the EPM approach for modeling flow and transport in a fractured aquifer. Our approach is similar to the one of Berkowitz et al., in that we consider a synthetic fracture network and use a numerical discrete fracture computer model to generate results against which to evaluate the EPM model predictions. However, the emphasis in this study is on validation of the EPM approach using effective transport parameters that are calculated directly from known fracture characteristics, rather than calibrated against tracer breakthrough or other transport results.

Although the lack of real world data for model validation is a primary motivation for considering a synthetic fracture system, the use of numerically generated flow and transport data has the advantage that it allows the evaluation of the EPM approach under a range of conditions and over different time scales. The problem considered here is necessarily a much simplified version of reality, but still the modelled system is designed to approximate a real world disposal site. The computer model used to perform the EPM model simulations is the VAM2D code (Huyakorn et al., 1989), but results of this study should apply to any porous medium flow and transport model. 


\section{METHODOLOGY}

\subsection{DESCRIPTION OF WASTE DISPOSAL SITE}

In this study we investigate the migration of solutes from a hypothetical waste disposal trench excavated in a fractured formation. The conceptual system is shown in cross-sectional view in Figure 2.1. Dimensions of the modeled domain and different material zones are depicted in this figure. The modeled cross-section measures $150 \mathrm{~m}$ in length $\times 20 \mathrm{~m}$ in height. The $10 \mathrm{~m}$ wide $\times 3 \mathrm{~m}$ deep disposal trench is located near the upper left corner of the domain and is capped with a low permeability clay cover. The hydraulic boundary conditions include constant hydraulic heads on the left and right boundaries, a fixed head upper boundary where the head value varies linearly from left to right, and a no-flow lower boundary condition. These boundary conditions impose steady state flow with a gradient pointing in the direction of the positive $\mathrm{x}$-axis. The assumption of steady state, uniformly saturated conditions is dictated by the use of the FRACTRAN computer code for generating contaminant plumes (see next section). Throughout this report, we consider fully saturated conditions. However, the analysis and conclusions regarding contaminant transport pertain equally well to steady state, unsaturated systems, provided the water saturation is constant in each porosity zone. In this case, porosity should be replaced by volumetric water content and saturated hydraulic conductivity should be replaced by unsaturated conductivity, with a value determined by ambient water content. Complications will arise however, in determining the hydraulic conductivity of fractures under partially saturated conditions. In general, it is expected that under unsaturated conditions, fractures may quickly be desaturated and as a result become barriers rather than conduits for flow and transport. While modeling flow and transport in variably saturated system is a problem of great practical significance, we do not consider this additional complication here, and restrict the analysis to a fully water-saturated system. However, the situation of (near) saturated conditions is not at all unrealistic for many actual, near-surface waste disposal sites in the northern United States and Canada (e.g. Grisak and Cherry, 1975; Cherry, 1989) upon which the problem considered here is loosely based. At many of these sites, disposal pits or trenches are excavated in low permeability glacial tills. These formations typically have very low matrix permeability. Relatively humid climatic conditions may result in saturated or near-saturated conditions, even at shallow depth, throughout much of the year. Bulk hydraulic and transport characteristics are often strongly influenced by the presence of fractures. The presence of fractures in such deposits has been attributed to a variety of mechanisms, including weathering, desiccation, post-glacial stress relief, shearing and tectonism (Vorauer et al., 1986). Typically, fracturing is most intensive near the soil surface, with fractures becoming sparser with depth. This fracture pattern is reproduced at our hypothetical site (Figure 2.2). The waste zone and clay cover are treated as non-fractured porous media. A fracture network is overlain on the rest of the model domain. We consider a regular and orthogonal fracture network consisting of four zones with a uniform fracture pattern in each zone. The upper fracture zone extends to a depth of 6 meters (elevation $14-20 \mathrm{~m}$ ). The fracture spacing is $2 \mathrm{~m}$ in the horizontal direction and $1 \mathrm{~m}$ in the vertical direction in the first fracture zone. All fractures in this zone have a uniform aperture of 60 microns $\left(610^{-5} \mathrm{~m}\right)$. The second fracture zone occurs between elevations of 10 


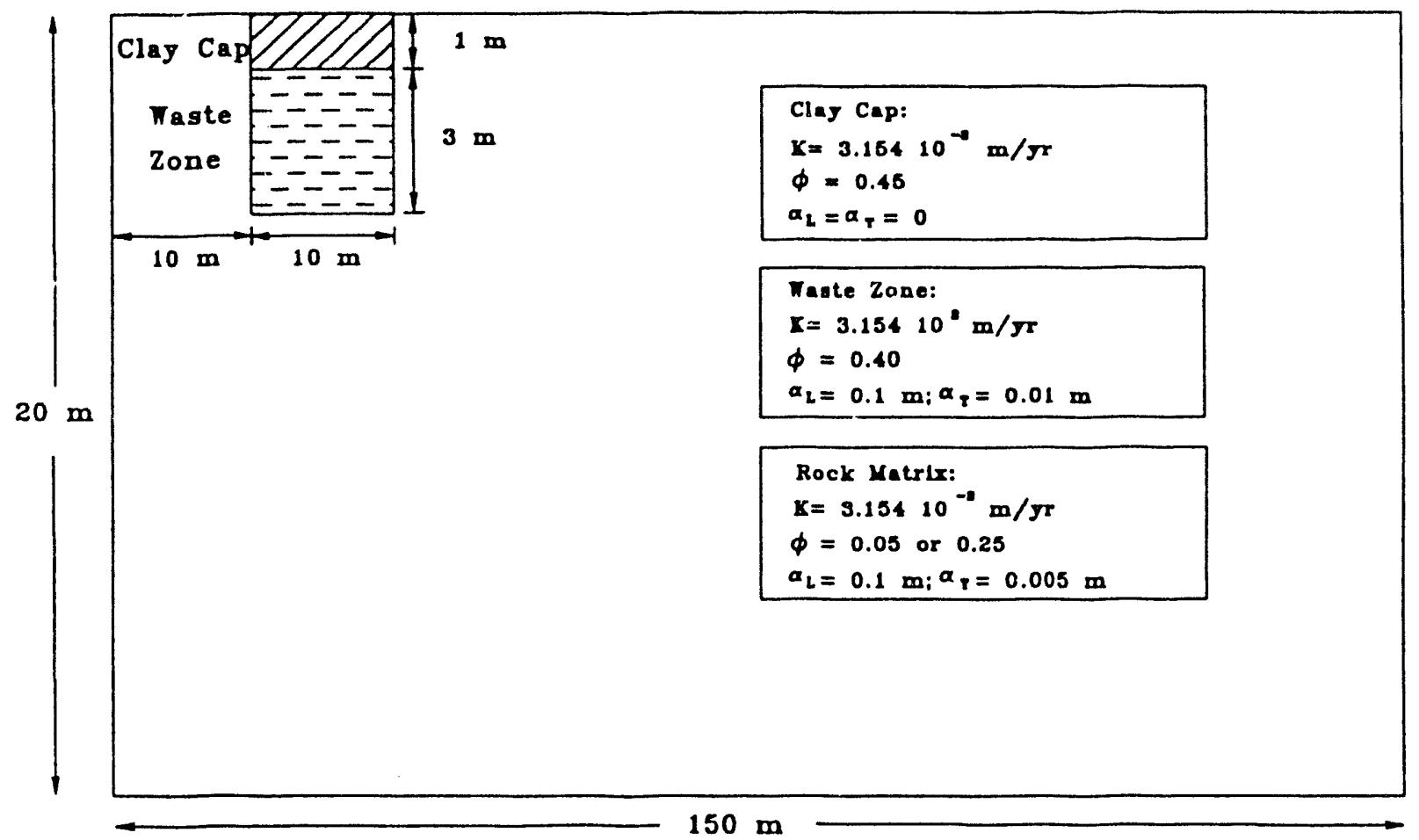

Figure 2.1. Schematic cross-sectional view of the hypothetical waste disposal site considered in this study. 
and $14 \mathrm{~m}$. The fracture spacing in this zone increases to $4 \mathrm{~m}$ and $2 \mathrm{~m}$ in the horizontal and vertical directions, respectively. The fracture aperture is reduced to $40 \mu \mathrm{m}$. The third zone occurs between elevations of $4 \mathrm{~m}$ and $10 \mathrm{~m}$. The frequency of vertical fracture in this zone is further reduced by a factor of 2 in this zone, resulting in fracture spacings in the horizontal and vertical directions of 8 and $2 \mathrm{~m}$, respectively. The fourth and deepest fracture zone shows a spacing between vertical fractures of $16 \mathrm{~m}$. Only a single horizontal fracture at the interface with the overlying zone is present. Fracture apertures in the last two zones are $20 \mu \mathrm{m}$. Figure 2.2 also shows that due to discontinuity between fracture zones, only a relatively few vertical fractures extend all the way downward across the model domain.

Two contaminant migration scenarios are considered. The first one involves a nonreactive solute species; the second involves a sorbing species. The first case is a worst-case where the retardation effect of sorption is ignored altogether. The second case involves a scenario where most sorption occurs inside the matrix and relatively little sorption on the fracture walls. Specific sorption parameters are presented in Section 3. In both scenarios it is assumed that the contaminant is initially present in the waste zone only, and is uniformly distributed at unit relative concentration. The contaminant is released from the waste zone by leaching and diffusion.

In order to model this site, we must make certain assumptions about the availability of site characterization data that would be required in an actual field situation. The most important assumption is that detailed information is available on the fracture characteristics, including knowledge on the vertical and horizontal fracture spacings and fracture apertures in the four different zones. It is also assumed that data is available, for instance from soil core analyses, on the hydraulic characteristics (hydraulic conductivity, porosity) of the clay cap, the waste zone and the porous matrix in the fractured zone. For non-conservative transport modeling, it is assumed that accurate estimates are available of distribution coefficients for both porous regions and fractures. Finally, we assume that piezometric data are available from which to assess vertical and horizontal hydraulic gradients. This latter information is used to determine the magnitude of seepage velocities which in turn are required to estimate effective dispersivities (see Section 2.3). 


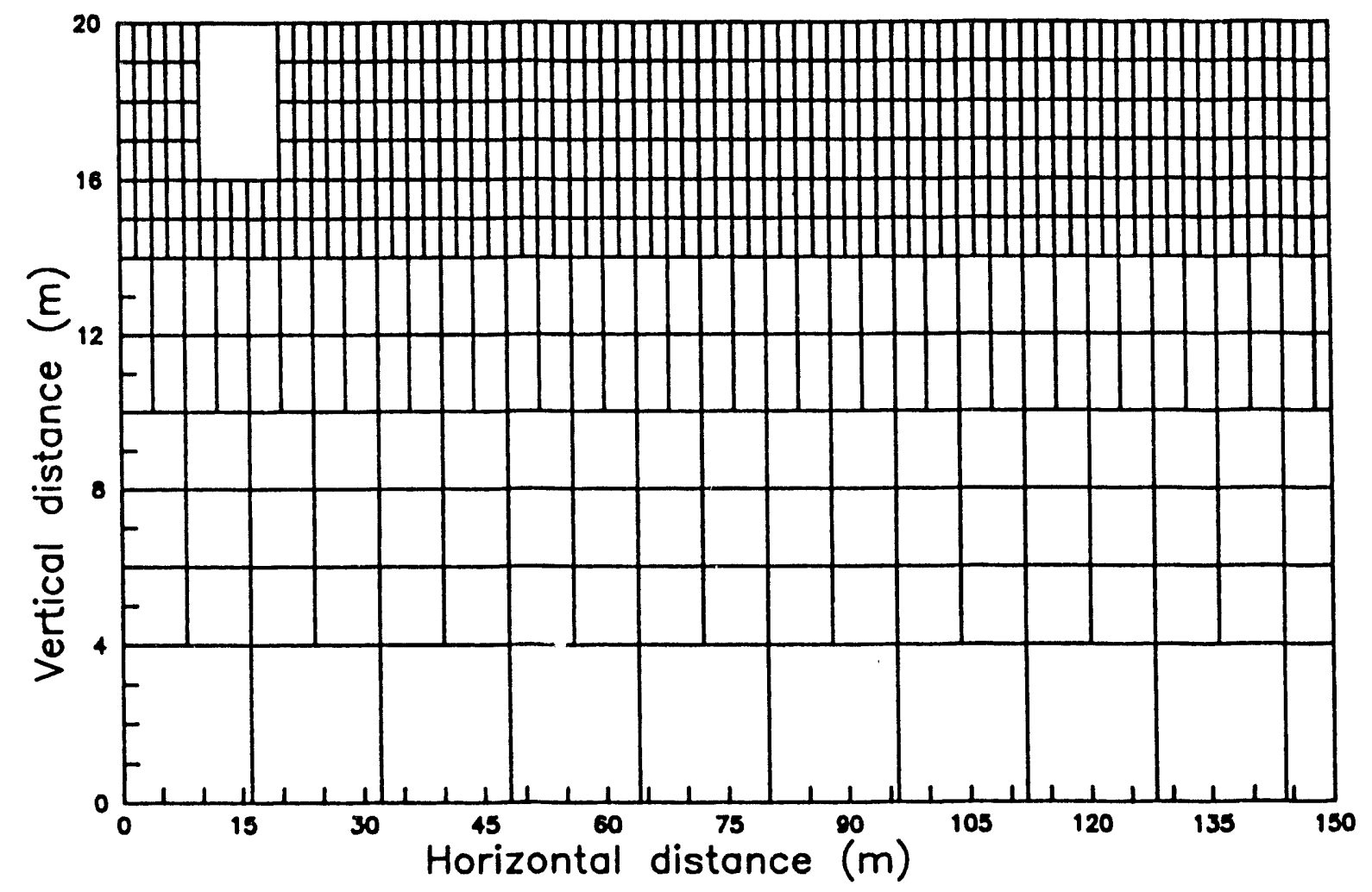

Figure 2.2. Fracture network at hypothetical waste disposal site. Note 5x vertical exaggeration. 


\subsection{FRACTRAN DISCRETE FRACTURE FLOW AND TRANSPORT MODEL}

\subsubsection{Model Formulation}

In this study, the FRACTRAN computer code (Sudicky, 1989b) is used to generate the 'true' groundwater flow field and transient contaminant plumes. FRACTRAN considers steady state fluid flow and transient solute transport in a two-dimensional, saturated, and nondeformable fractured porous medium. The present version of the model can consider either regular or irregular discrete fracture networks, where the fracture orientations are aligned with the cartesian coordinate system. The numerical model solves the flow and transport equations pertaining to fractures and porous matrix with appropriate continuity condition at the matrix-fracture interface. The flow and transport model is analogous to the one considered by Berkowitz et al., (1988).

For a porous matrix block with hydraulic conductivity $\mathrm{K}_{\mathrm{ij}}$, the steady-state groundwater flow equation is (Bear, 1972):

$$
\frac{\partial}{\partial x_{i}} K_{i j} \frac{\partial h}{\partial x_{j}}=0 \quad(i=1,2)
$$

where $\mathrm{h}=\psi+\mathrm{y}$ is the hydraulic head, where $\psi$ is the pressure head and $\mathrm{y}$ is the elevation above datum and the $x_{i}(i=1,2)$ are cartesian coordinates. Here $x_{1} \equiv x$ is taken to represent the horizontal direction; $x_{2} \equiv y$ represents the vertical direction. The fluid flow rate, $\mathrm{V}_{\mathrm{i}}$, at any point within the porous matrix is defined by the Darcy equation:

$$
v_{i}=-K_{i j} \frac{\partial h}{\partial x_{j}}
$$

Considering a system of planar fractures with constant aperture $2 b$ (see Fig. 2.4), and a uniform hydraulic head across the fracture width, then steady flow within a fracture intersecting a matrix block can be described using (see e.g., Berkowitz et al., 1988):

$$
2 b K_{f} \frac{\partial^{2} h_{f}}{\partial \ell^{2}}-\left.V_{n}\right|_{I^{-}}+\left.V_{n}\right|_{l^{+}}=0
$$

where $h_{f}$ is the hydraulic head in the fracture, $\ell$ is the distance along the fracture and $K_{f}$ is the fracture hydraulic conductivity given by (Snow, 1969):

$$
K_{f}=\frac{(2 b)^{2} \rho g}{12 \mu}
$$

where $\rho$ is the fluid density, $\mathrm{g}$ is the gravitational constant, and $\mu$ is the dynamic viscosity of the fluid. The last two terms on the left-hand side of (3) represent the normal components of the fluid leakage flux ( $\left(\mathrm{I}^{-}\right.$and $\mathrm{I}^{+}$) across the bounding interfaces that separate the fracture and porous 
matrix. This flux term along with an assumed continuity in head (i.e., $h=h_{f}$ ) along the matrixfracture interface provides the link between equations (1) and (3). By combining (4) with the one-dimensional form of the Darcy equation (2), the fluid flow rate, $v_{f}$, along the fracture axis is given by:

$$
V_{f}=-K_{f} \frac{\partial h_{f}}{\partial \ell}
$$

Transport of dissolved substances in the matrix-fracture system is described in a manner analogous to the flow problem, with separate transport equations for matrix and fractures. The formulation of FRACTRAN allows for first-order degradation (e.g., radionuclide decay) and equilibrium sorption onto fracture walls and solid phase of the porous matrix. In the present study, decay reactions are not considered and are omitted from the governing equations. The coupling between the matrix and fracture transport equations is provided by the continuity of concentration along the interface between the two regions combined with equality of the normal component of the solute mass flux across the matrix-fracture interface. Transport in the porous matrix is described by the advection-dispersion equation (Bear, 1972):

$$
\frac{\partial}{\partial x_{i}}\left(\phi_{m} D_{m i j} \frac{\partial c}{\partial x_{j}}\right)-V_{i} \frac{\partial c}{\partial x_{i}}=\phi_{m} R_{m} \frac{\partial c}{\partial t}
$$

where $c$ is the solute concentration, $\phi_{m}$ is the matrix porosity, the $D_{\text {mij }}$ are components of the dispersion tensor, $R_{m}$ is the matrix retardation factor, and $t$ is time.

Solute transport in a fluid-filled fracture is described by (e.g., Tang et al., 1981):

$$
2 b\left[\frac{\partial}{\partial \ell} D_{f} \frac{\partial c_{f}}{\partial \ell}-V_{f} \frac{\partial c_{f}}{\partial \ell}\right]=2 b\left[R_{f} \frac{\partial c_{f}}{\partial t}+\left.\Gamma\right|_{I^{-}}-\left.\Gamma\right|_{I^{+}}\right]
$$

where $c_{f}$ is the concentration in a fracture, $R_{f}$ is the fracture retardation coefficient and $V_{f}$ and $D_{f}$ are the flow rate and longitudinal hydrodynamic dispersion coefficient in the fractur:, respectively. $D_{f}$ is given by (Tang et al., 1981):

$$
D_{f}=\alpha_{f} V_{f}+D_{m}
$$

where $\alpha_{\mathrm{f}}$ is a longitudinal dispersivity, and $\mathrm{D}_{\mathrm{n}}$ is a molecular diffusion coefficient. Even in a smooth-walled fracture, hydrodynamic dispersion will occur as a result of velocity variations along the fracture cross-section (Taylor, 1953). The terms involving $\Gamma$ in (7) represent the loss (or gain) of solute mass across the fracture-matrix interface due to fluid leakage and hydrodynamic dispersion. For example, along interface $I^{*}$, the flux term is written as:

where $V_{n}$ is the normal component of the Darcy flux, and $D_{n}$ and $\partial c / \partial n$ are the dispersion coefficient and concentration gradient, respectively, acting perpendicular to the fracture-matrix 


$$
\left.\Gamma\right|_{I^{-}}=\left[V_{n} c-\phi D_{n} \frac{\partial c}{\partial n}\right]
$$

interface $\mathrm{I}^{-}$. In ac'dition, the continuity condition $c_{\mathrm{m}}=\mathrm{c}_{\mathrm{f}}$ along the fracture interface is used in coupling (6) and (7).

\subsubsection{Solution Technique}

The numerical solution of the governing equations (1) and (3) for steady state flow, and (6) and (7) for transient solute transport are obtained using the Laplace Transform Galerkin (LTG) method (Sudicky, 1989). In this method, standard Galerkin finite element techniques are applied for spatial discretization; however, the time derivatives appearing in the transport equations (6) and (7) are first removed by applying the Laplace transformation to these terms. Details of the procedure are described by Sudicky (1989a). The LTG method is very efficient for long term simulations, since it yields a solution that is conunuous in time and does not involve the use of time-stepping to advance the solution through time. It has been shown by Sudicky (1989) that the LTG scheme yields highly accurate solutions even when a relatively coarse (i.e., coarse relative to local Peclet numbers) spatial discretization is used. This is particularly important for the problem at hand because large concentration gradients can develop at matrix-fracture interfaces. The avoidance of time steps, and the corresponding avoidance of Courant number constraints involved in conventional time stepping schemes, is also very beneficial because extremely large ground water velocity contrasts can exist between the porous matrix and the fractures.

The spatial discretization used here for the porous matrix is comprised of rectangular elements. The fracture network is represented by superimposing a number of one-dimensional line elements representing individual fracture segments onto the rectangular element mesh. By super-imposing the fracture line elements onto the rectangular porous matrix grid, the equality of hydraulic head and concentration at the matrix-fracture interface is automatically accommodated. Moreover, upon element assembly, the fluid and mass exchange terms in the flow and transport equations are accounted for naturally such that explicit calculation of the terms involving $V_{n}$ in (3) and $\Gamma$ in (7) is unnecessary.

In order to obtain a high degree of accuracy in resolving head and concentration differences between fractures and matrix, a detailed finite element grid was employed in the FRACTRAN modeling of the waste disposal site depicted in Figure 2.1. A total of 27,600 elements with average dimensions of $0.5 \mathrm{~m}$ by $0.21 \mathrm{~m}$ were used to represent the porous matrix, while 6625 line elements were used to model the individual fractures shown in Figure 2.2. This discretization resulted in the use of a total of 27,993 nodal points. The large number of nodal points involved in the FRACTRAN simulations demonstrates the substantial computer resources, including in this case approximately 20 megabytes of random access memory, required for detailed modeling of fractured groundwater systems. 


\subsection{EPM APPROXIMATIONS FOR FLOW AND TRANSPORT MODELING}

Modeling of flow and contaminant transport from the hypothetical waste disposal site using the EPM approach is accomplished using the VAM2D computer code (Huyakorn et al., 1989). Details of the formulation and capabilities of VAM2D are discussed elsewhere; here we focus on the determination of effective flow and transport parameters. In an equivalent porous medium (EPM) model, Darcy's law, see Eq. (1), is assumed to describe global flow through the combined system of fractures and porous matrix. No distinction is made between hydraulic head in matrix and fractures and the hydraulic conductivities now become equivalent (or effective) parameters.

In order to model flow using the porous medium approximation it is necessary to first determine the components of the equivalent hydraulic conductivity, $\mathrm{K}_{\mathrm{ij}}$. Approximations for the equivalent hydraulic conductivity of fracture networks have been presented by Snow (1969), Maini and Hocking (1977) and others. The fracture network considered in this study consists of two parallel sets of fractures, with four different fracture zones (Figure 2.2). Maini and Hocking (1977; referenced by de Marsily, 1986) have presented the following relationships for determining the directions of anisotropy and the principal axes of the effective hydraulic conductivity for two-dimensional, parallel fracture networks:

$$
\begin{gathered}
\epsilon_{i}=\frac{1}{2} \arctan \left(\frac{\sin 2 \theta}{\cos 2 \theta K_{a} / K_{b}}\right) \\
K_{i}=\frac{K_{a} K_{b} \sin ^{2} \theta}{K_{a} \sin ^{2} \epsilon_{i}+K_{b} \sin \left(\theta-\epsilon_{i}\right)}
\end{gathered}
$$

where $\theta$ is the angle between the fracture sets, $\epsilon_{i}(i=1,2)$ is the angle of the $i$-th axis of anisotropy and $\mathrm{K}_{\mathrm{a}}$ and $\mathrm{K}_{\mathrm{b}}$ are the equivalent directional hydraulic conductivities of the two sets of fractures, see Figure 2.3.

In the present case, the angle $\theta$ equals $90^{\circ}$ which produces the simple result of:

$\epsilon_{1}=0, \epsilon_{2}=90^{\circ}, \mathrm{K}_{11}=\mathrm{K}_{\mathrm{a}}, \mathrm{K}_{22}=\mathrm{K}_{\mathrm{b}}$. In other words, this approximation indicates that vertical fractures do not contribute to the hydraulic conductivity in the horizontal direction, and vice-versa. The principal axes of anisotropy are also aligned with the cartesian coordinate system so that cross-components in the equivalent hydraulic tensor are zero. The hydraulic conductivity tensor, $[\mathrm{K}]$, can thus be written as:

$$
[K]=\left(\begin{array}{cc}
K_{11} & 0 \\
0 & K_{22}
\end{array}\right)
$$




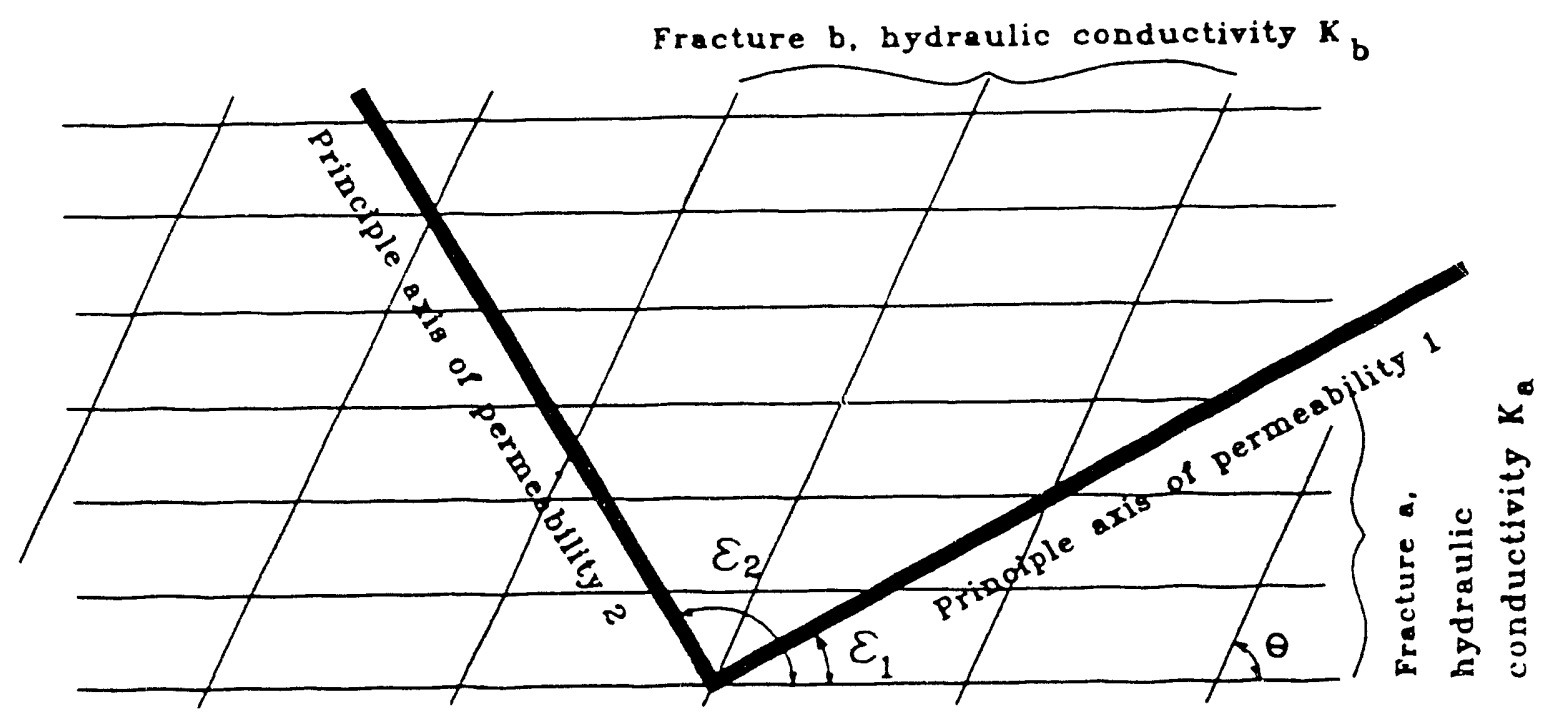

Figure 2.3. Orientation of the principal axes of anisotropy in a fracture network consisting of two sets of parallel fractures (After Maini and Hocking, 1977; reproduced with permission of the authors). 
If effects of the flow in the perpendicular direction are negligible, flow in the direction parallel to fractures in a fracture and matrix system becomes equivalent to flow through a parallel layer system. Therefore, component coefficients of the equivalent hydraulic conductivity in Eq. (12) can be calculated from the parallel layer model (Bear, 1972):

$$
\begin{gathered}
K_{i i}=\frac{1}{L_{i}}\left[b K_{f}+\left(L_{i}-b\right) K_{m}\right] \\
(i=1,2)
\end{gathered}
$$

where $L_{i}$ is half spacing of fractures in the $x_{i}$ direction, $b$ is half aperture of fractures in the $x_{i}$ direction, as shown in Figure 2.4, and $\mathrm{K}_{\mathrm{f}}$ and $\mathrm{K}_{\mathrm{m}}$ are hydraulic conductivities of fracture and matrix, respectively. The former is given by Eq. (9).

In many cases, the matrix hydraulic conductivity, $\mathrm{K}_{\mathrm{m}}$, is many orders of magnitude smaller than the fracture conductivity and the last term in (13) is often dropped. Equations (4) and (13) can then be combined to:

$$
K_{i i}=\text { constant } \cdot b^{3}
$$

which is the familiar 'cubic law' of flow through fractured media.

The transport equation upon which the EPM modeling is based is the advectiondispersion equation, see Eq. 6, which is now written for the combined matrix-fracture system as

$$
\frac{\partial}{\partial x_{i}}\left(\phi D_{i j} \frac{\partial c}{\partial x_{j}}\right)-V_{i} \frac{\partial c}{\partial x_{i}}=\phi R \frac{\partial c}{\partial t}
$$

The transport equation has the same form as (6), except that the parameters $\phi, D, V$ and $R$ are now effective parameters whose values depend on specific fracture and matrix characteristics. Sources, sinks and decay processes are ignored in (15). The dispersion coefficients $D_{i j}$ incorporate the effects of molecular diffusion and hydrodynamic mixing due to micro-scale variations in the porewater velocity. VAM2D employs the usual assumption (Bear, 1961) that hydrodynamic dispersion is linearly proportional to the average pore water velocity, leading to the following constitutive relations (Scheidegger, 1961):

$$
\phi D_{11}=\frac{\alpha_{L}\left(V_{1}\right)^{2}}{|V|}+\frac{\alpha_{r} \cdot\left(V_{2}\right)^{2}}{|V|}+D^{*}
$$




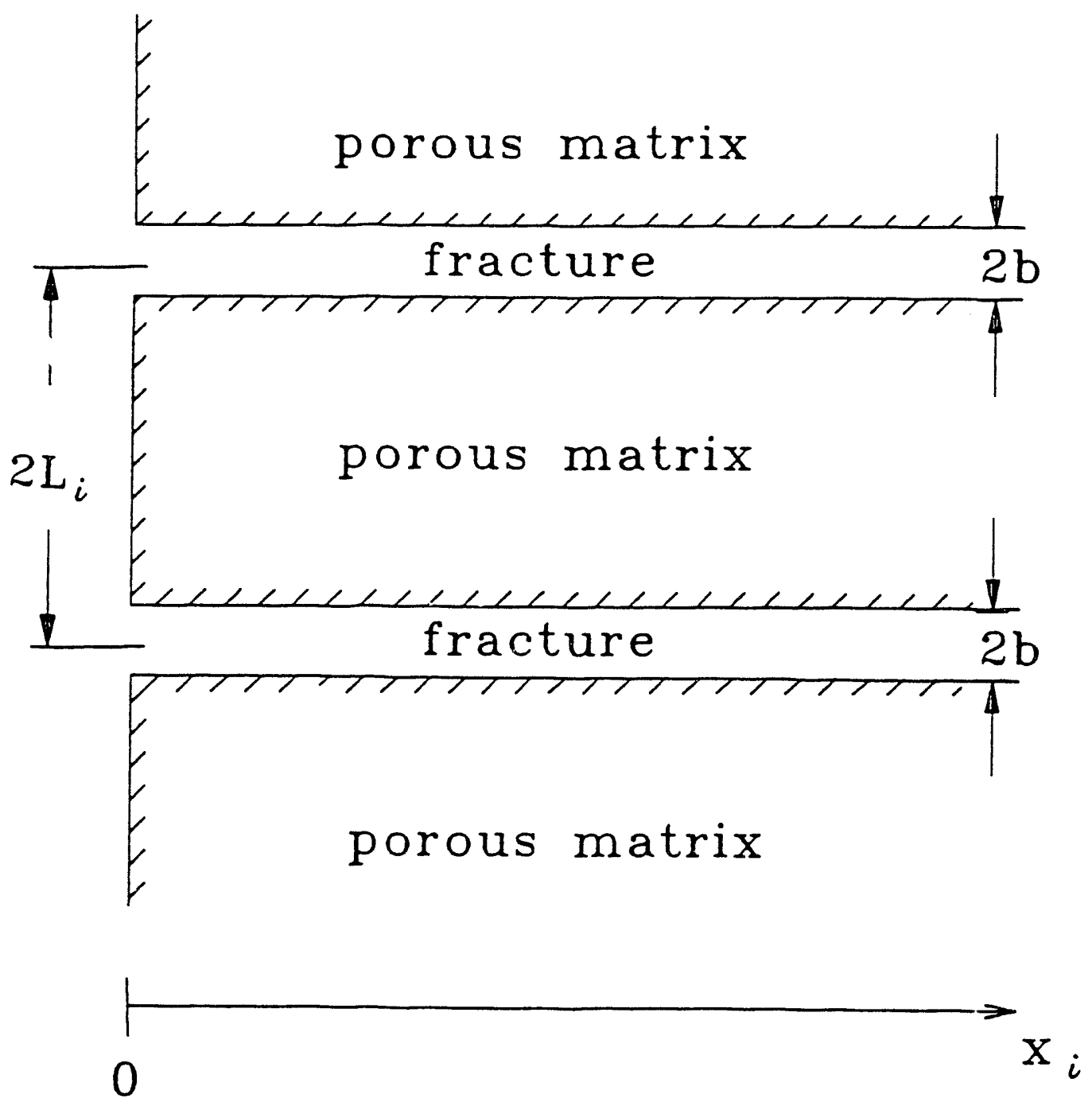

Figure 2.4. Schematic of parallel fracture system. 


$$
\begin{aligned}
& \phi D_{22}=\frac{\alpha_{L}\left(V_{2}\right)^{2}}{|V|}+\frac{\alpha_{T}\left(V_{1}\right)^{2}}{|V|}+D^{*} \\
& \phi D_{12}=\phi D_{21}=\left(\alpha_{L}-\alpha_{T}\right) \frac{V_{1} V_{2}}{|V|}
\end{aligned}
$$

where $\alpha_{\mathrm{L}}$ and $\alpha_{\mathrm{T}}$ are longitudinal and transverse dispersivities, respectively, $|\mathrm{V}|$ is the absolute value of Darcy's velocity, and $D^{*}$ is the apparent molecular diffusion in the water-phase of the porous medium. Eqs. (16) - (18) are used in VAM2D with the apparent molecular diffusion coefficient of the water-phase computed by the code using the following relation:

$$
D^{*}=\left(\phi S_{w}^{n}\right) D_{w}
$$

where $D_{w}$ is the free-water molecular diffusion coefficient, $S_{w}$ is the water saturation, and $n$ is an exponent parameter with a value of $n=1.333$.

The velocity components $\mathrm{V}_{\mathrm{i}}$ are obtained by solving the flow equation as outlined above. Determination of the effective dispersivities is one of the most critical steps in the application of the EPM approach. The presence of fractures will lead to a large contrast in pore water velocity between matrix and fracture. In the EPM approach, this velocity contrast is treated as an apparent dispersion process. In a computer simulation this is reflected in large values assigned to the dispersivity parameters. As mentioned in the introduction, several authors have presented expressions for the effective EPM model dispersion coefficients of a fractured site. Such expressions may be derived in several different ways, based on the comparison between the EPM model and a more rigorous matrix-fracture transport model. Methods that have been employed include a more or less empirical approach based on shape factors (van Genuchten, 1985), time moment analysis (Parker and Valocchi, 1986) and application of Laplace transform techniques (van Genuchten and Dalton, 1986). van Genuchten and Dalton (1986) presented the following expression for the equivalent longitudinal dispersion coefficient, $D_{e}$, for a system of parallel fractures separated by rectangular matrix blocks during steady state unidirectional flow:

$$
D_{e}=\frac{D_{f} \phi_{f}}{\phi_{T}}+\frac{\phi_{m}(L-b)^{2} v^{2} R_{m}^{2}}{3 \phi_{T} D_{m}^{*} R_{e}^{2}}
$$

where $D_{f}$ is the dispersion coefficient in fracture, $v_{e}=V / \phi_{T}$ is the seepage velocity averaged over fractures and matrix, $\phi_{\mathrm{m}}, \phi_{\mathrm{f}}$ and $\phi_{\mathrm{T}}$ are the matrix, fracture and total porosity, respectively, $\mathrm{L}$ is the fracture half spacing, $\mathrm{D}_{\mathrm{m}}^{*}$ is the apparent diffusion coefficient in the matrix, $R_{m}$ is the matrix retardation factor and $R_{e}$ is the total retardation factor of the system, defined as:

where $R_{f}$ is the retardation factor in fracture and $\phi_{T}$ is simply the sum of matrix and fracture porosity: 


$$
\begin{gathered}
R_{e}=\frac{\phi_{f} R_{f}+\phi_{m} R_{m}}{\phi_{T}} \\
\phi_{T}=\phi_{m}+\phi_{f}
\end{gathered}
$$

For the orthogonal system of parallel fractures considered in this study, the fracture porosity is given by:

$$
\phi_{f}=\frac{b\left(L_{1}+L_{2}\right)}{L_{1} L_{2}}
$$

where $L_{1}$ and $L_{2}$ are the half-spacings between fractures in the horizontal and vertical directions, respectively.

The contribution due to dispersion inside the fractures can be determined from the parallel plate model (Dullien, 1979):

$$
D_{f}=\frac{2}{105} \frac{b^{2} v_{f}^{2}}{D_{w}}
$$

where $v_{f}$ is the mean velocity in the fracture. Since for most fractured media, $\phi_{\mathrm{f}}<<\phi_{\mathrm{m}}$, $b<<(L-b)$, and $R_{m} / R_{e} \geq 1$, the effect of dispersion inside the fractures can be safely ignored, allowing (20) to be siriplified to:

$$
D_{e} \approx \frac{\phi_{m}(L-b)^{2}}{3 \phi_{T} D_{m}^{*}}\left(\frac{R_{m}}{R_{e}}\right)^{2} v_{e}^{2}
$$

For the EPM approximation of the fractured-matrix system, Eq. (25) can be written in terms of the Darcy velocity, $\mathrm{V}$, as:

$$
\phi_{T} D_{e}=\alpha_{e}|V|
$$

where the equivalent longitudinal dispersivity $\alpha_{e}$ is given by 


$$
\alpha_{e}=\frac{\phi_{m}(L-b)^{2}}{3 \phi_{T}^{2} D_{m}^{w}}\left(\frac{R_{m}}{R_{e}}\right)^{2}|V|=\text { constant } \cdot|V|
$$

In the present study, the above analysis for one-dimensional transport has been extended to a two-dimension transport problem in an anisotropic fracture-matrix system. Since groundwater flow at the hypothetical waste site is dominantly horizontal, the longitudinal dispersivity $\alpha_{\mathrm{L}}$ is calculated as:

$$
\alpha_{L}=\frac{\phi_{m}\left(L_{1}-b\right)^{2}}{3 \phi_{T}^{2} D_{m}^{w}}\left(\frac{R_{m}}{R_{e}}\right)^{2}\left|\bar{V}_{1}\right|
$$

where $\bar{V}_{1}$ is the average Darcy's velocity component in the horizontal direction, and the transverse dispersivity is calculated as:

$$
\alpha_{T}=\frac{\phi_{m}\left(L_{2}-b\right)^{2}}{3 \phi_{T}^{2} D_{m}^{w}}\left(\frac{R_{m}}{R_{e}}\right)^{2}\left|\bar{V}_{2}\right|
$$

where $\bar{V}_{2}$ is the average Darcy's velocity component in the vertical direction.

Expressions analogous to (20) have been developed for certain other fracture geometries, and are summarized by van Genuchten and Dalton (1986). They all show a quadratic relationship between the dispersion coefficient and velocity, instead of the linear relationship for true porous media. A common assumption underlying the derivation of these effective dispersion coefficients is that in the actual fracture-matrix system, advection occurs only in the fracture and that movement in the matrix is by diffusion only. In our case, the true system as modeled with the FRACTRAN code, does include flow through the matrix. This contribution is ignored in the approximation of the effective transport parameters, on the notion that the effects due to fractures are much greater than the effect of advective flow through the matrix.

Using the equations presented in this section it is possible to compute the EPM model input parameters required for the VAM2D simulations. Due to the dependence on fracture geometry and apertures, the effective flow and transport parameters must be calculated for each fracture zone. To accommodate the variation of fracture spacing and aperture with depth, the fractured region is divided into four horizontal layers in the VAM2D modeling. These layers are numbered from the lower domain boundary upward and coincide with the different fracture regions depicted in Figure 2.1. The vertical extent of each layer is as follows: 


$$
\begin{aligned}
& 0<\text { Layer } 1 \leq 4 \mathrm{~m} \\
& 4 \mathrm{~m} \text { Layer } 2 \leq 10 \mathrm{~m} \\
& 10 \mathrm{~m} \text { Layer } 3 \leq 14 \mathrm{~m} \\
& 14 \mathrm{~m} \text { Layer } 4 \leq 20 \mathrm{~m}
\end{aligned}
$$

Effective flow and transport parameters are calculated separately for each of the layers using the procedures outlined in the previous section.

\subsection{FINITE ELEMENT DISCRETIZATION FOR EPM MODELING}

For the VAM2D modeling of flow and transport, the two-dimensional domain depicted in Figure 2.1 is discretized into a rectangular finite element grid. The grid is shown in Figure 2.5. A total of 1768 elements are used in constructing this grid, with average horizontal and vertical element dimensions of $2.8 \mathrm{~m}$ and $0.6 \mathrm{~m}$ respectively. This grid is thus considerably coarser than the one used for the FRACTRAN simulations. The motivation to use the selected grid is based on considerations applicable to modeling actual field sites. First, the grid must be sufficiently small to produce a desired level of accuracy in results. On the other hand, a very fine grid such as the one used here for the FRACTRAN code, is usually not warranted due to lack of very detailed site characterization data and restrictions imposed by available computer resources. When transport simulations are to be performed, an important consideration in determining grid size, is the Peclet number constraint which says that element dimensions should not exceed 3 or 4 times the dispersivity. This criterion was employed in designing the VAM2D grid, with dispersivity in this case referring to the effective dispersivity. 


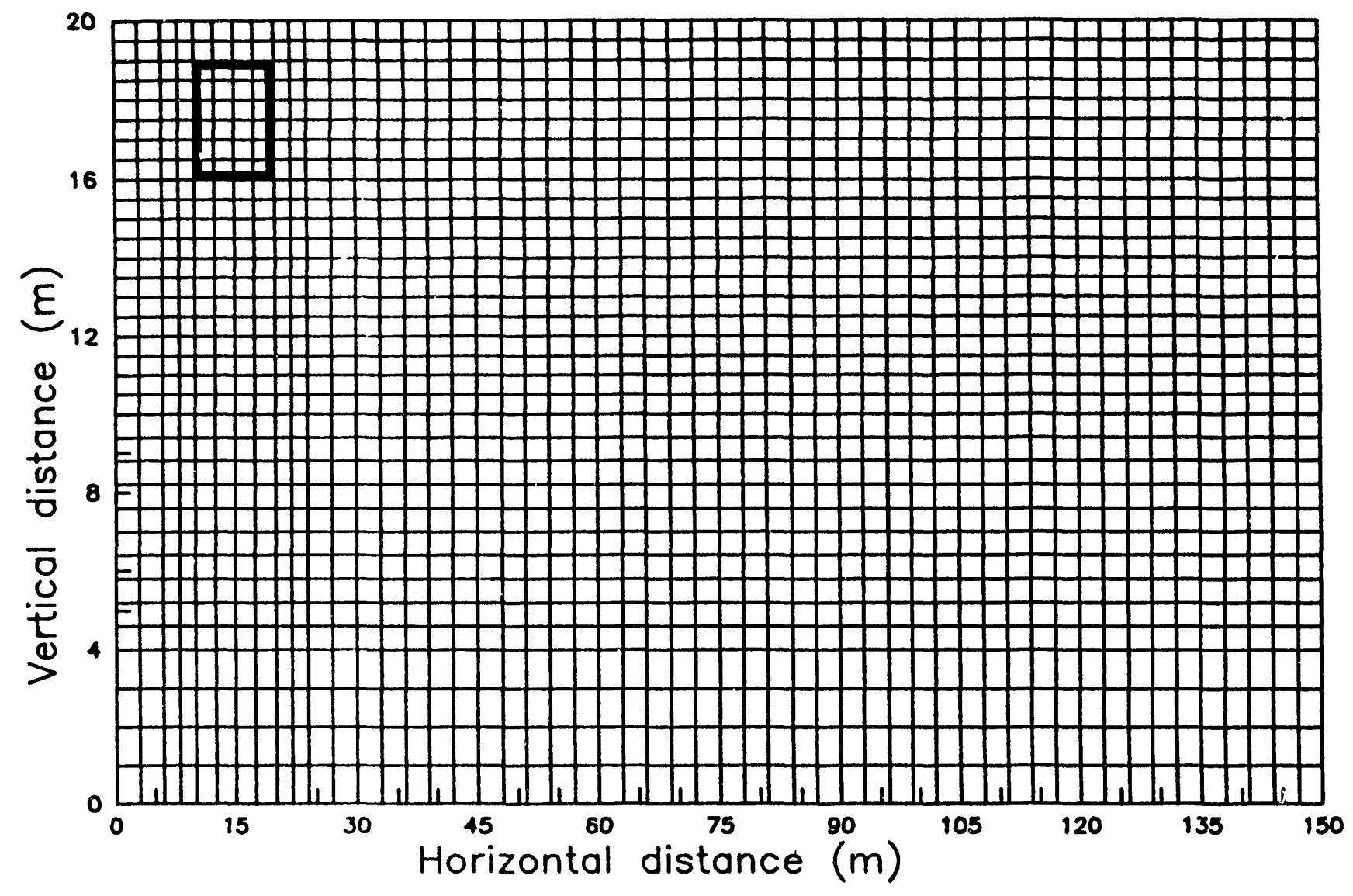

Figure 2.5. VAM2D finite element grid used in EPM modeling. 


\section{RESULTS}

\subsection{FLOW MODELING}

The first step in the modeling procedure is to simulate hydraulic head and velocity distributions in the flow region. As shown in Figure 2.1, the two-dimensional flow system consists of fracture network, matrix blocks, waste disposal zone and clay cap. The waste and clay zones will be treated as porous media, and fracture and matrix will be approximated by the EPM continuum approach as discussed in the previous section. The system is fully saturated with water and steady-state flow is assumed to remain in the whole simulation period of interest.

Parameters relating to fracture spacing, aperture, porosity and hydraulic conductivity are summarized in Table 3.1. The components of hydraulic conductivity of fractures in the vertical and horizontal directions are calculated using Eq. (4); the porosity of fractures in each layer is determined from equation (23). The properties for the porous matrix, clay cap and waste zone, are given in Table 3.2. Parameters for the flow simulation in the EPM approach are provided in Table 3.3., with equivalent hydraulic conductivities and total porosities for each layer calculated using Eqs. (13) and (22), respectively. The boundary conditions of the twodimensional steady-state flow system are shown in Figure 3.1. On the left-hand side boundary, a constant hydraulic head $(\mathrm{h}=20 \mathrm{~m})$ is kept, and a linearly varying head boundary is imposed on the top surface. The downstream right-hand side boundary is separated into two parts, the upper portion $(18 \mathrm{~m} \leq \mathrm{y} \leq 20 \mathrm{~m})$ is a seepage face with zero pressure head $(\psi=0)$, and the lower portion $(0 \leq \mathrm{y} \leq 18 \mathrm{~m})$ is subject to a constant hydraulic head $(\mathrm{h}=18 \mathrm{~m})$.

The steady state flow modeling results are presented in Figure 3.2 which shows the hydraulic head distribution predicted by VAM2D in comparison with the 'true', i.e., FRACTRAN computed head distribution. It can be seen that the agreement is quite close, indicating that the EPM approximations are effective for describing steady state flow in this fractured system. This result also indicates that the EPM approach should be effective in describing the advective transport component in subsequent contaminant transport analyses.

\subsection{TRANSPORT MODELING}

\subsubsection{Transport Scenarios}

In most real world situations, accurate assessment of contaminant migration is of more concern than groundwater flow prediction. It is also expected that inadequacies of the EPM approach will be revealed more readily in errors in transport predictions than in flow simulation results, especially for the steady state flow conditions considered here. We therefore evaluate three different transport scenarics:

1. Conservative contaminant with low matrix porosity $\left(\phi_{\mathrm{m}}=0.05\right)$.

2. Conservative contaminant with medium matrix porosity $\left(\phi_{\mathrm{m}}=\right.$ $0.25)$. 
Table 3.1. Geometric and Hydraulic Fracture Properties of the Modeled System

\begin{tabular}{||c||c|c|c|c|c||}
\hline Parameter & Direction & Layer 1 & Layer 2 & Layer 3 & Layer 4 \\
\cline { 2 - 6 } & Horizontal & 20 & 20 & 40 & 60 \\
\cline { 2 - 6 } & Vertical & 20 & 20 & 40 & 60 \\
\hline \multirow{2}{*}{ Spacing (2L) (m) } & Horizontal & 4 & 2 & 2 & 1 \\
\cline { 2 - 6 } & Vertical & 16 & 8 & 4 & 2 \\
\hline $\begin{array}{c}\text { Hydraulic } \\
\text { Conductivity (m/y) }\end{array}$ & Horizontal & $2,578.01$ & $2,578.01$ & $41,248.08$ & $92,810.44$ \\
\cline { 2 - 7 } & Vertical & $2,578.08$ & $2,578.01$ & $41,248.08$ & $92,810.44$ \\
\hline \multicolumn{2}{|c|}{ Porosity $\left(\phi_{\mathrm{f}}\right)$} & $6.2 \times 10^{-6}$ & $1.25 \times 10^{-5}$ & $3 \times 10^{-5}$ & $9 \times 10^{-5}$ \\
\hline
\end{tabular}

* In calculation of hydraulic conductivity, $\rho=1,000 \mathrm{~kg} / \mathrm{m}^{3}$ and $\mu=1 \mathrm{cp}=31,536(\mathrm{~kg} / \mathrm{m} \cdot \mathrm{y})$. 
Table 3.2. Porous Medium Properties of the Modeled System

\begin{tabular}{|c|c|c|c|}
\hline Parameter & Matrix & Clay Cap & Waste Zone \\
\hline Porosity & 0.05 or 0.25 & 0.45 & 0.40 \\
\hline $\begin{array}{c}\text { Hydraulic } \\
\text { Conductivity }(\mathrm{m} / \mathrm{y})\end{array}$ & $3.1536 \times 10^{-2}$ & $3.1536 \times 10^{-3}$ & $3.1536 \times 10^{2}$ \\
\hline
\end{tabular}

Table 3.3. Effective Hydraulic Parameters Used in the EPM Flow Modeling.

\begin{tabular}{|c|c|c|c|c|c|c|c|}
\hline \multicolumn{2}{|l|}{ Parameter } & Layer 1 & Layer 2 & Layer 3 & Layer 4 & Clay Cap & $\begin{array}{l}\text { Waste } \\
\text { Zone }\end{array}$ \\
\hline \multicolumn{2}{|c|}{ Material Number } & 1 & 2 & 3 & 4 & 5 & 6 \\
\hline \multirow{2}{*}{$\begin{array}{l}\text { Equivalent } \\
\text { Hydraulic } \\
\text { Conduct- } \\
\text { ivity }(\mathrm{m} / \mathrm{y})\end{array}$} & Horizontal & 0.044426 & 0.057316 & 0.856490 & 5.60010 & 0.003154 & 315.360 \\
\hline & Vertical & 0.034758 & 0.037891 & 0.444016 & 2.81585 & 0.003154 & 315.360 \\
\hline
\end{tabular}




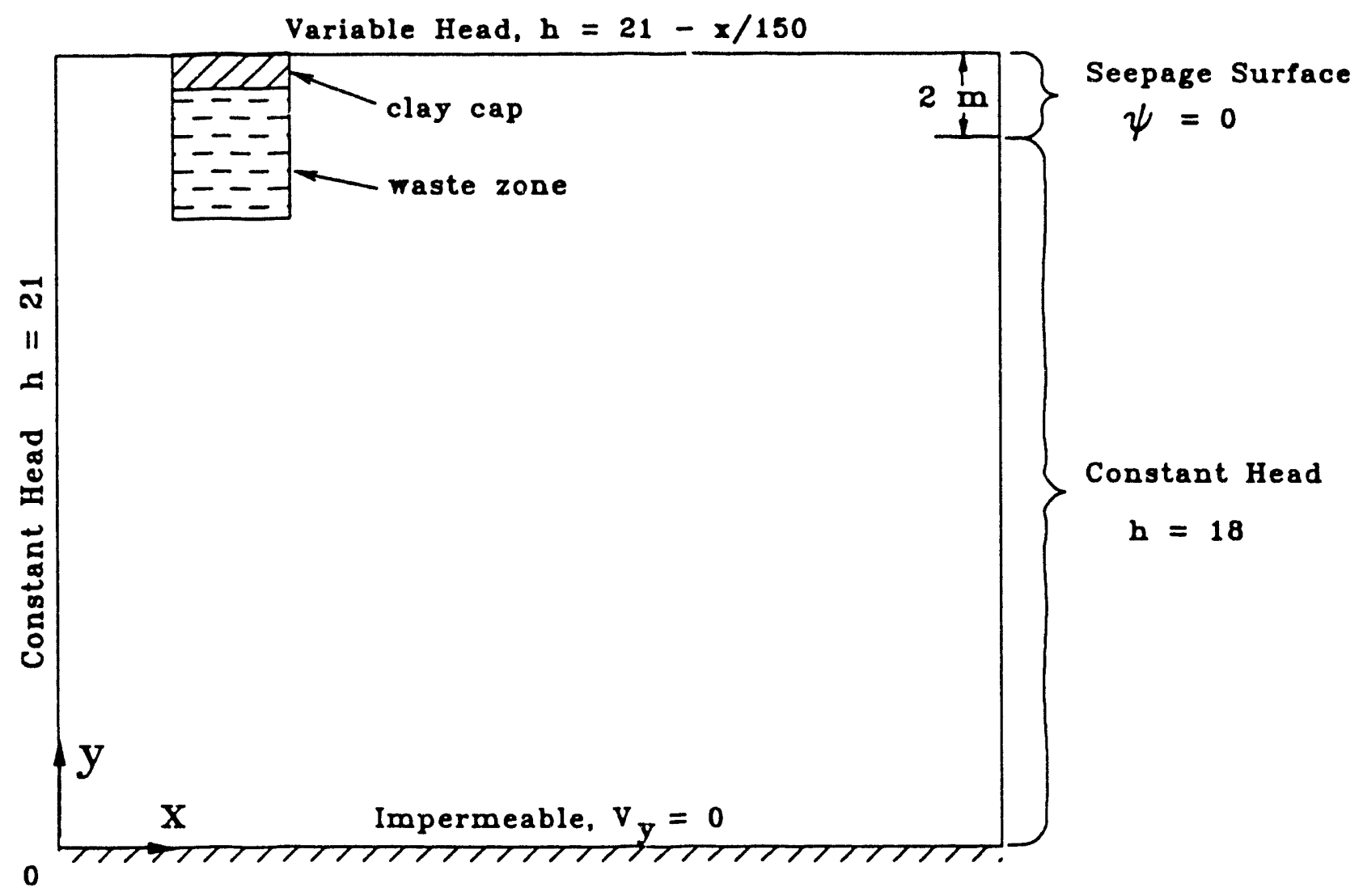

Figure 3.1. Boundary conditions and coordinates used in the EPM simulation of steady-state flow. 


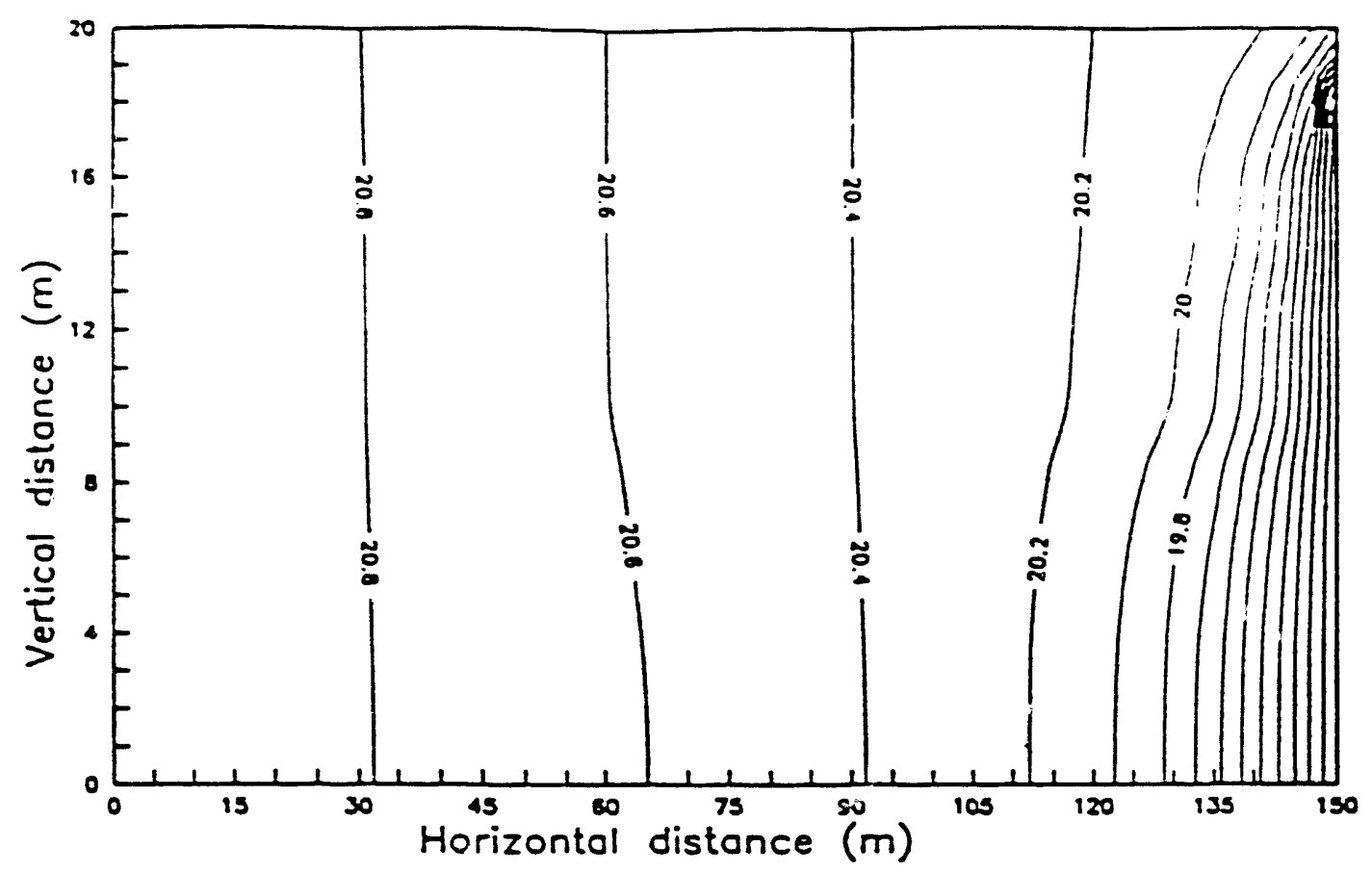

(a)

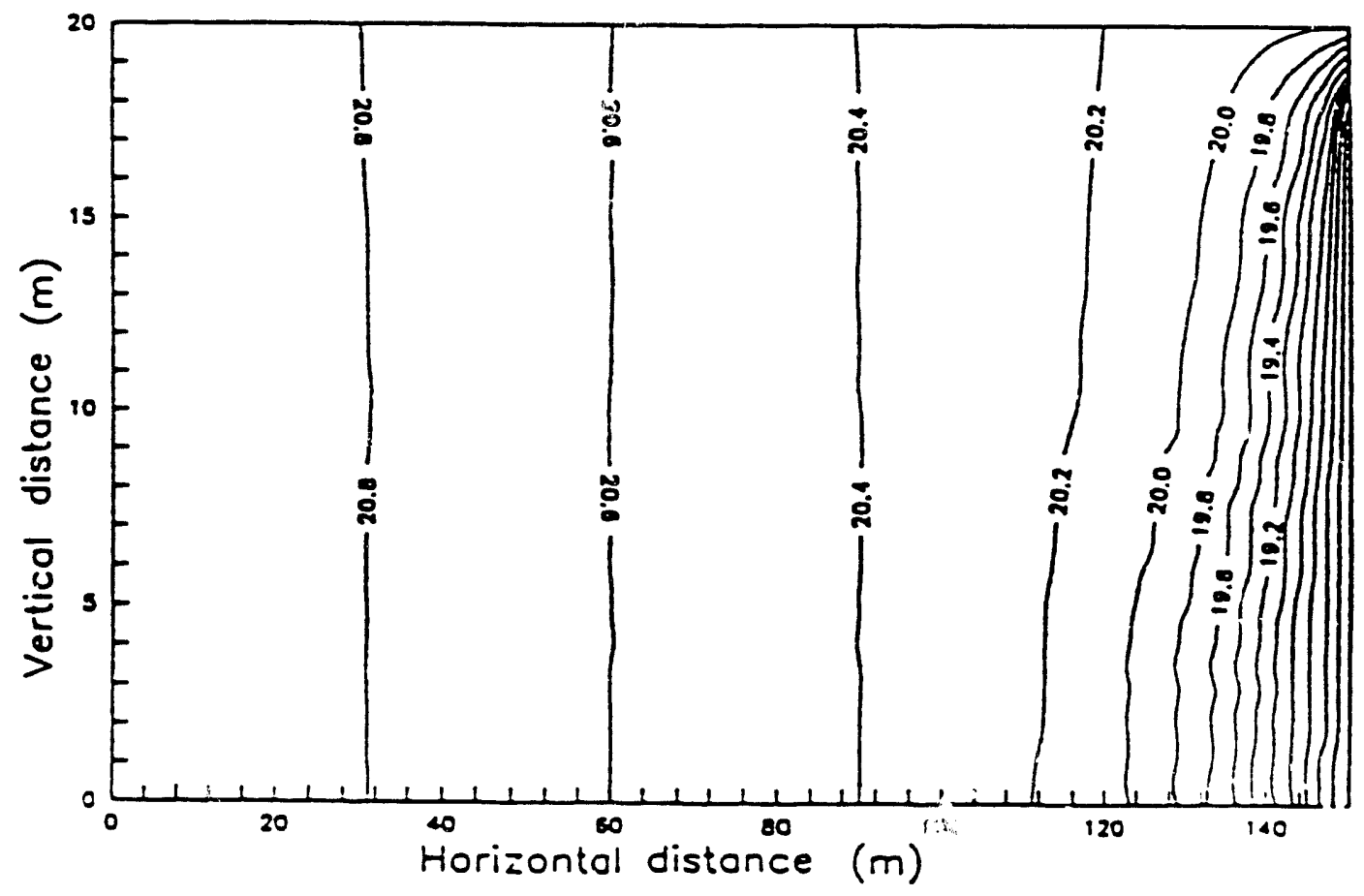

(b)

Figure 3.2. Steady-state hydraulic head distributions in the model domain computed by FRACTRAN (a) and VAM2D (b). 
3. Reactive contaminant with different retardation factors for fracture and matrix.

Results for these different scenarios are presented in the following sections.

\subsubsection{Conservative Contaminant Transport}

In conservative contaminant modeling, the only transport mechanisms involved are advection, diffusion and dispersion; reaction effects due to (radioactive) decay and adsorption, are ignored. In this case, the retardation factors in matrix, fracture, clay cap and waste zone are all set to one, so $R_{e}=1$ in Eq. (15). Figure 3.2 shows that the hydraulic head gradients, and therefore flow velocities in the system are very uniform except in the region near the left boundary. This suggests that it is possible to use average Darcy velocity components to estimate the longitudinal and transverse dispersivities for the transport calculations using Eqs. (28) and (29). Average $x$ - and $y$ - velocity values are determined by multiplying effective hydraulic conductivity and average hydraulic gradient, with the latter estimated directly from Figure 3.2. The procedure followed is analogous to a practical situation where direct measurements of velocity are rot available and the required velocity values would instead be estimated form hydraulic conductivities and gradient. Provided the fracture system is well characterized, the effective hydraulic conductivity can be calculated as outlined in Section 2, while the hydraulic gradient would typically be estimated from piezometer data. The resulting average velocity values and dispersivities used in the simulation are shown in Table 3.4. Note that the dispersivity coefficients are quite sensitive to the matrix porosity change from $\phi_{\mathrm{m}}=0.5$ to $\phi_{\mathrm{m}}$ $=0.25$. The overall porosity, $\phi_{\mathrm{T}}$, is essentially the same as the matrix porosity for both cases. The contribution of the fractures to the overall porosity is negligible, even in the most densely fractured upper layer.

The boundary and initial conditions for both conservative and reactive contaminant transport are shown in Figure 3.3. On the left-hand side and top boundaries, a Dirichlet type boundary condition with constant concentration $c=0$ is specified. On the bottom and righthand side boundaries, a Neumann boundary condition is posed with zero normal concentration gradients. The time period for the transport simulation is chosen such that the boundary conditions on the downstream boundaries will not have much effects on the prediction results. The initial condition of the system, as shown in Figure 3.3., is:

$$
c(x, y, t=0)= \begin{cases}1(10 \leq x \leq 20,16 \leq y \leq 19) \\ 0 & \text { otherwise }\end{cases}
$$

i.e., there is non-zero concentration distribution initially only in the waste zone.

With the top boundary representing a watertable, a physically more meaningful boundary condition would be obtained by prescribing a zero contaminant mass-flux condition along the upper model boundary. A zero concentration condition is used instead since the version of the FRACTRAN code used in this study permits only first-type or second-type boundary conditions, 


\begin{tabular}{|c|c|c|c|c|c|c|c|c|c|c|}
\hline & 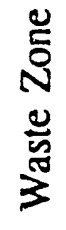 & 0 & 1 & 1 & $\overline{0}$ & ర్ & $\overrightarrow{0}$ & $\stackrel{\sigma}{0}$ & $\stackrel{\text { f }}{\circ}$ & ๖ \\
\hline . & $\begin{array}{l}\text { जे } \\
\frac{\pi}{0}\end{array}$ & $n$ & ' & ' & ơ & $\stackrel{n}{0}$ & $\begin{array}{l}8 \\
8 \\
0\end{array}$ & $\begin{array}{l}\text { ஜे } \\
\dot{0}\end{array}$ & $\stackrel{n}{\sharp}$ & 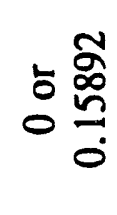 \\
\hline 总 & 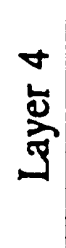 & $\forall$ & $\begin{array}{l}\tilde{0} \\
\frac{x}{0} \\
\frac{0}{a} \\
\dot{m}\end{array}$ & 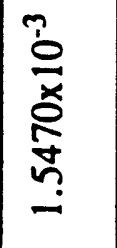 & 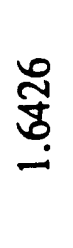 & $\begin{array}{l}0 \\
\text { ลิ } \\
\text { ஸ் }\end{array}$ & 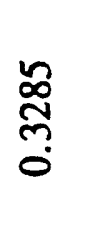 & $\begin{array}{l}\frac{a}{5} \\
\stackrel{0}{0} \\
\stackrel{0}{0}\end{array}$ & 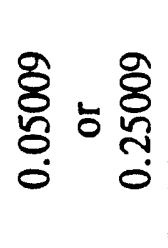 & $\frac{\stackrel{\aleph}{\infty}}{\stackrel{0}{0}}$ \\
\hline$\sum_{\sum}^{\frac{\infty}{D}}$ & 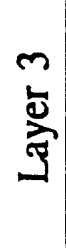 & $m$ & 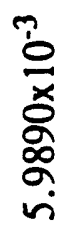 & 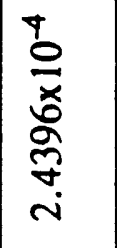 & $\underbrace{\infty}_{i}$ & ֻั & 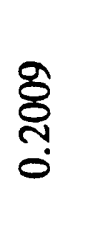 & $\begin{array}{l}\bar{z} \\
5 \\
0\end{array}$ & 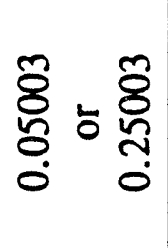 & $\frac{\widetilde{\delta}}{\stackrel{0}{\sigma}}$ \\
\hline 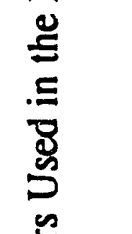 & 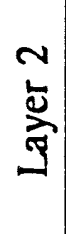 & $\sim$ & $\begin{array}{l}\frac{+}{0} \\
\frac{x}{\sigma} \\
\infty \\
\delta \\
\dot{0}\end{array}$ & $\begin{array}{l}n \\
0 \\
x \\
\alpha \\
0 \\
0 \\
0 \\
0 \\
\end{array}$ & $\begin{array}{l}\stackrel{\mathbb{S}}{\delta} \\
\stackrel{0}{0}\end{array}$ & $\begin{array}{l}\underset{+}{\infty} \\
0 \\
0\end{array}$ & $\begin{array}{l}\frac{n}{\sigma} \\
0 \\
0\end{array}$ & $\frac{n}{0}$ & 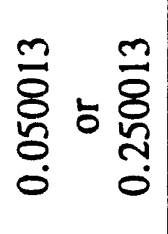 & $\frac{\tilde{\sigma}}{\stackrel{\infty}{\sigma}}$ \\
\hline 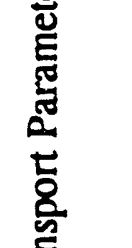 & \begin{tabular}{l}
- \\
\multirow{2}{*}{}
\end{tabular} & - & $\begin{array}{l}\frac{7}{x} \\
\frac{0}{6} \\
\frac{5}{2}\end{array}$ & 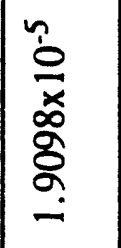 & 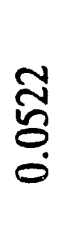 & 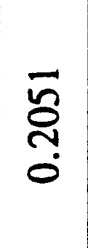 & $\begin{array}{l}\text { J } \\
\stackrel{0}{0} \\
\dot{0}\end{array}$ & $\begin{array}{l}\stackrel{0}{\Xi} \\
\stackrel{0}{0}\end{array}$ & 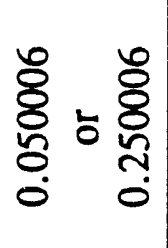 & $\frac{\widetilde{\Omega}}{\infty}$ \\
\hline 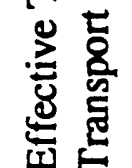 & $\frac{\bar{\Phi}}{\mathcal{U}}$ & 总 & $1>^{x}$ & $1>^{2}$ & $\vec{\sigma}$ & 5 & $\vec{\sigma}$ & tั & \multirow{2}{*}{ 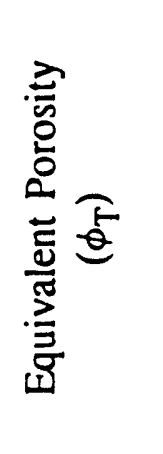 } & \multirow{2}{*}{ 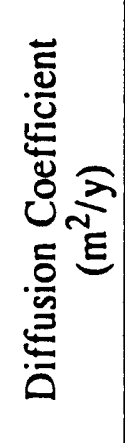 } \\
\hline$\stackrel{\dot{\sim}}{\ddot{\nu}}$ & 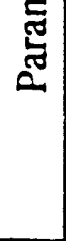 & . & \multicolumn{2}{|c|}{ 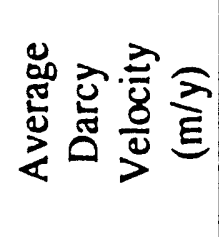 } & \multicolumn{2}{|c|}{ 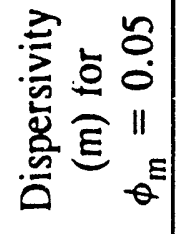 } & \multicolumn{2}{|c|}{ 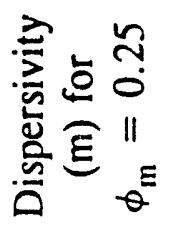 } & & \\
\hline
\end{tabular}




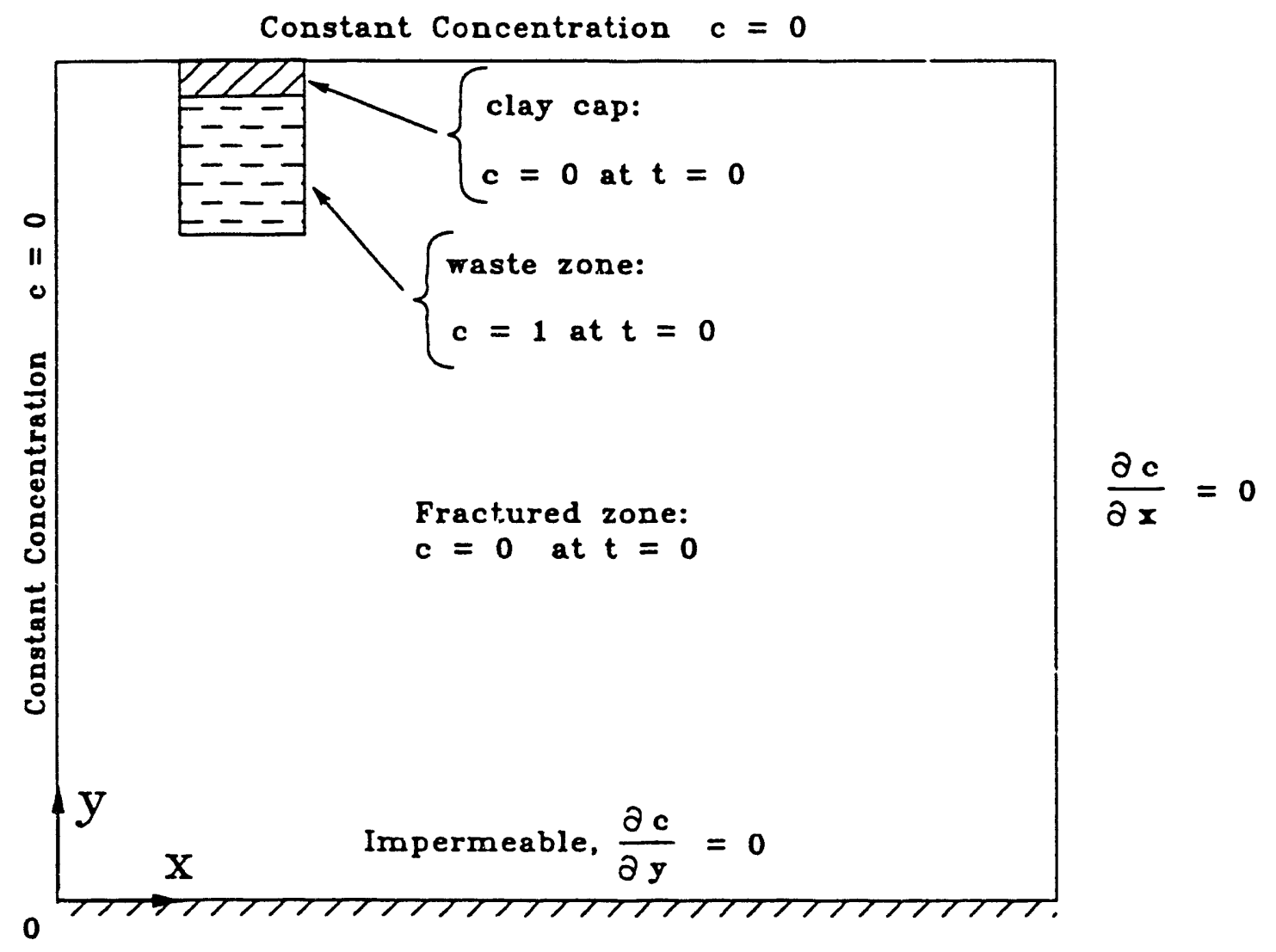

Figure 3.3. Boundary and initial conditions used in the VAM2D simulation of conservative and reactive contaminant transport. 
but not third-type conditions (i.e., prescribed mass-flux). However, since the fluid flow rates across the top boundary are quite low, and the dispersivity values used in the FRACTRAN modeling are also quite small, the FRACTRAN simulations approximate a zero flux boundary condition quite well. On the other hand, the effective dispersivities used in the VAM2D modeling are much higher than the FRACTRAN values with the result that a number of the VAM2D simulations slowed significant mass loss due to back dispersion across the top boundary, which in turn degrades the agreement between VAM2D and FRACTRAN transport results. The spurious back-mixing that may occur in EPM approximations of fractured groundwater systems has been mentioned also by others as one of the drawbacks of this approach (Berkowitz et al, 1988).

The comparison of concentration contours between VAM2D and the 'true' FRACTRAN results for the two cases of conservative contaminant transport is presented in Figures 3.4 - 3.7. Figures 3.4 and 3.5 show results after simulation times of $t=50$ years and $t=150$ years for the low matrix porosity case, $\phi_{\mathrm{m}}=0.05$. The FRACTRAN results are overlain on a graph of the fracture network. The concentration contours exhibit a jaggedness that reflects the influence of individual fractures on contaminant transport. In contrast, the VAM2D simulations show the typical smooth contours expected from a porous medium simulation. Some discrepancies between VAM2D and FRACTRAN occur near the source, which is not unexpected. The magnitude of the effective dispersivity in VAM2D allows some of the contaminant to disperse to the upstream (left) side of the disposal trench. VAM2D also shows the presence of contaminant in the clay cap which does not occur in the FRACTRAN simulation. This is an artifact of allowing dispersion into the clay layer in the VAM2D simulation. This was done to avoid certain numerical difficulties, whereas as in the 'true' FRACTRAN case the clay layer dispersivity was set to zero. At $\mathrm{t}=50$ years (Figure 3.4), the VAM2D and FRACTRAN results agree quite well; VAM2D accurately predicts the extent of the plume as represented by the 0.005 relative concentration contour, as well as the position of the concentration peak. A c'oser inspection of the VAM2D and FRACTRAN indicates some mass loss in the VAM2D result. This is shown most clearly in the smaller area enclosed by the 0.25 relative concentration contour, and is a result of the spurious back dispersion across the model top boundary mentioned above. At $t=150$ years (Figure 3.5) VAM2D again predicts the extent of the plume quite well, but the loss of mass is even more evident at this later time, resulting in under prediction of the magnitude of the concentration peak as well as an erroneous prediction of the position of the peak. The effect of the upper boundary is clearly evident in Figure 3.5, and illustrates that not only the determination of effective flow and transport parameters affects the accuracy of the EPM approximation, but that results may also be quite sensitive to the selected boundary conditions.

Results for the medium matrix porosity case, $\phi_{\mathrm{m}}=0.25$, are depicted in Figures 3.6 and 3.7. To facilitate comparison with the first case, concentration contours are plotted again for simulation times of $t=50$ and $t=150$ years. Comparison with the previous figures shows the quite dramatic effect of matrix porosity on the overall rate of plume migration. For the same Darcy flow rate, a higher porosity will result in enhanced diffusion from the fractures into the matrix, which acts as a retardation process. In the " $/ 1 \mathrm{M} 2 \mathrm{D}$ modeling this is approximated as 


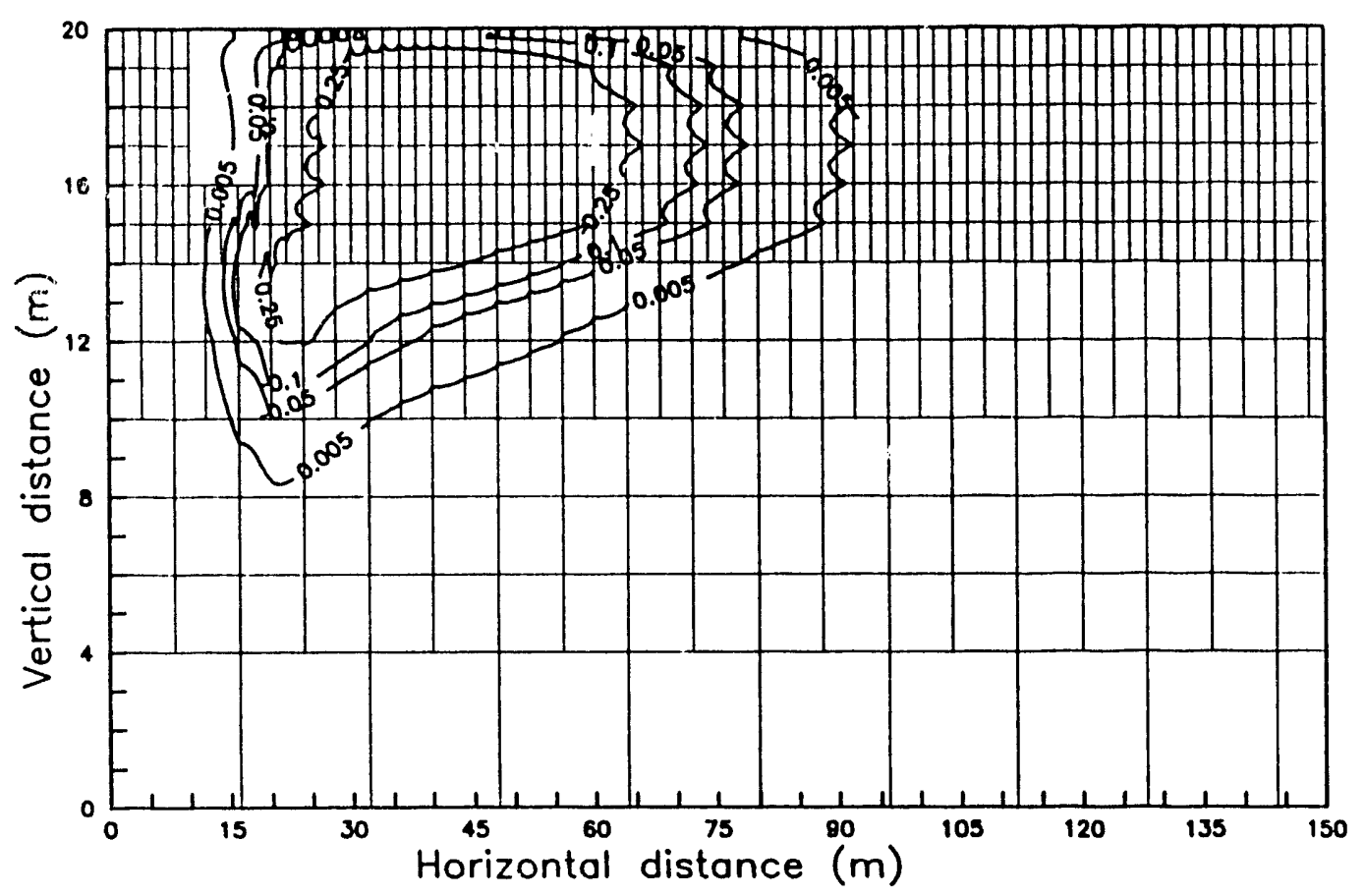

(a)

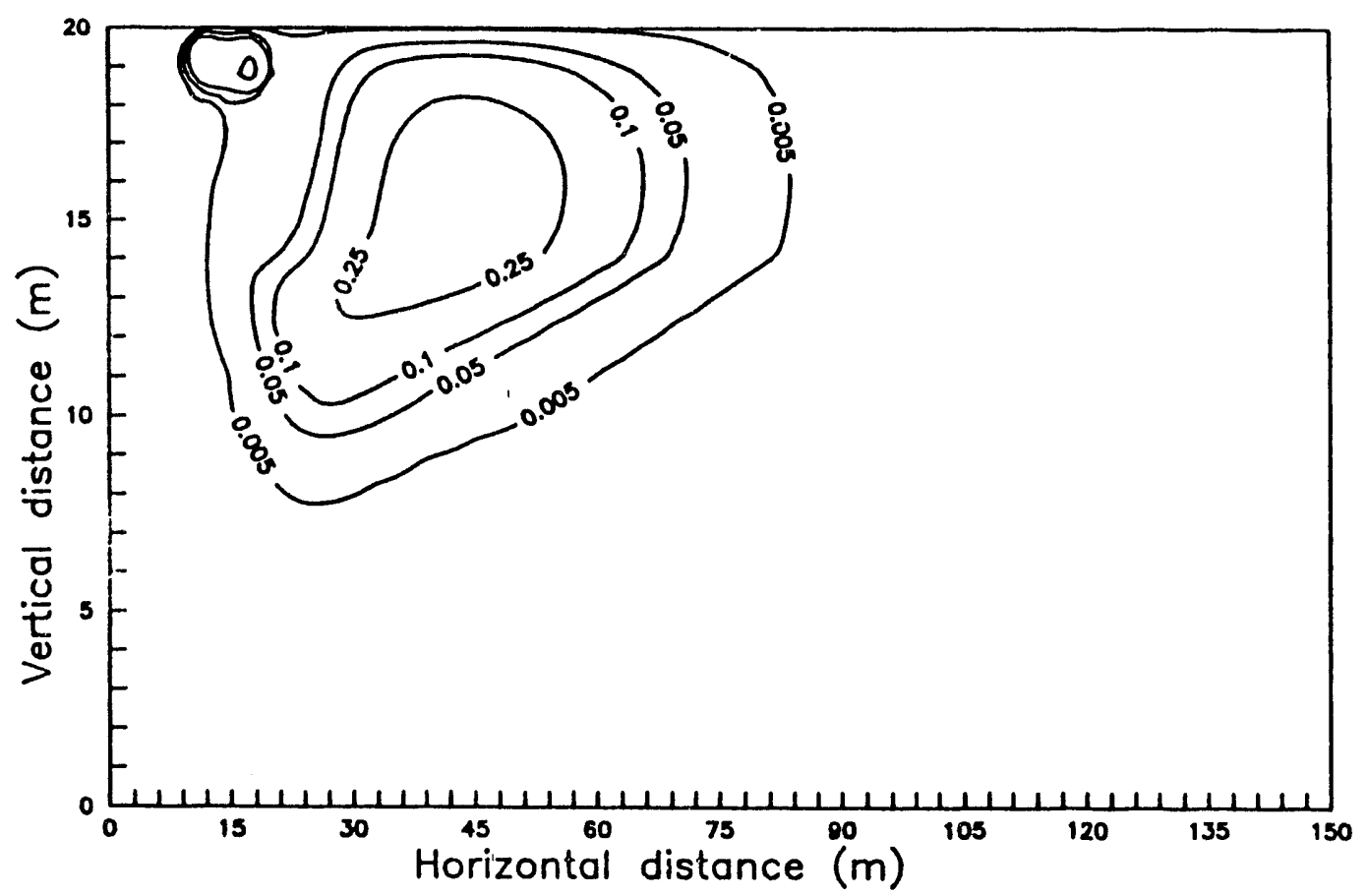

(b)

Figure 3.4. Concentration contours of conservative contaminant transport at $t$ $=50 \mathrm{y}$ with $\phi_{\mathrm{m}}=0.05$ as simulated by FRACTRAN (a) and VAM2D (b). 


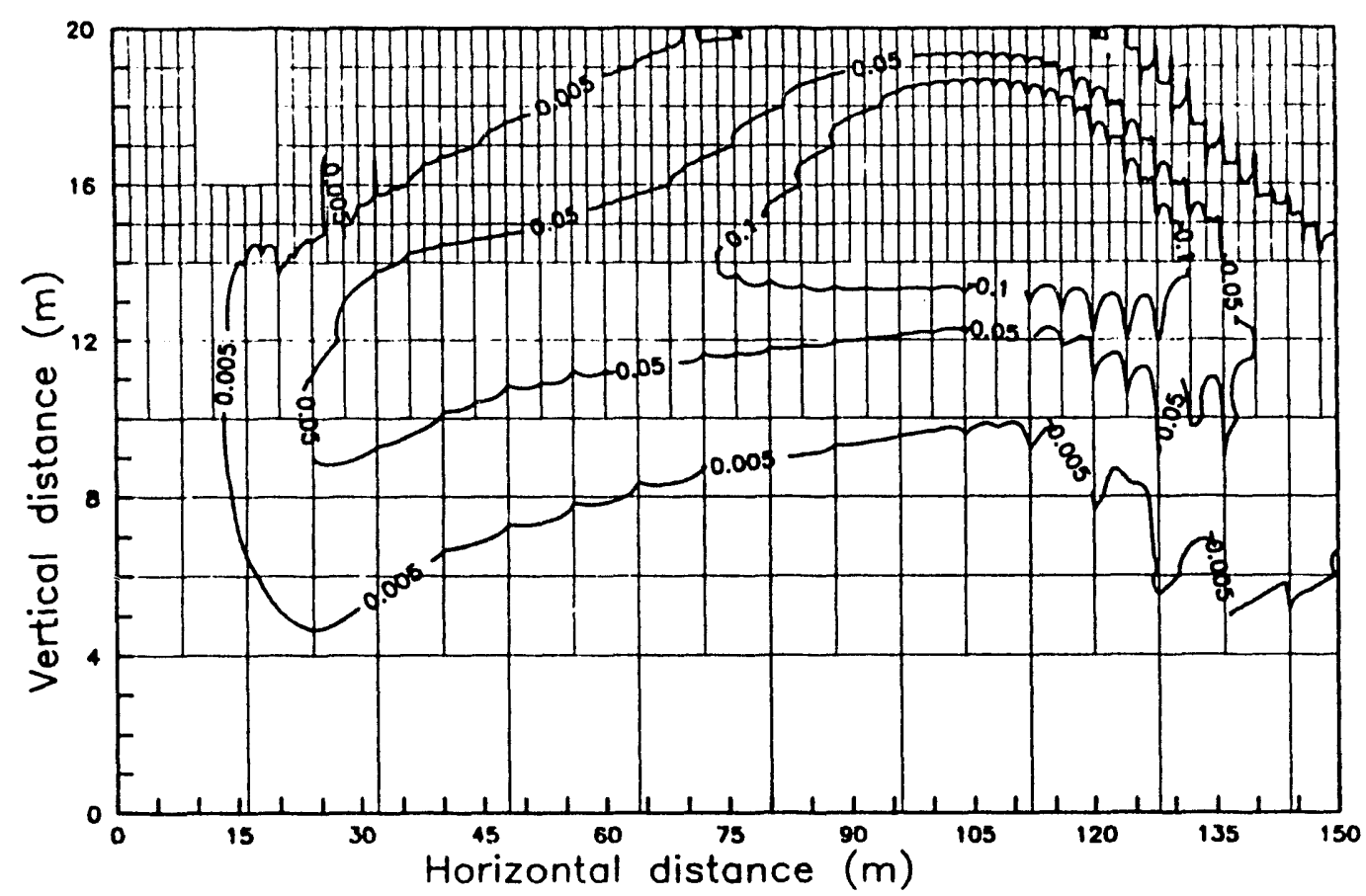

(a)

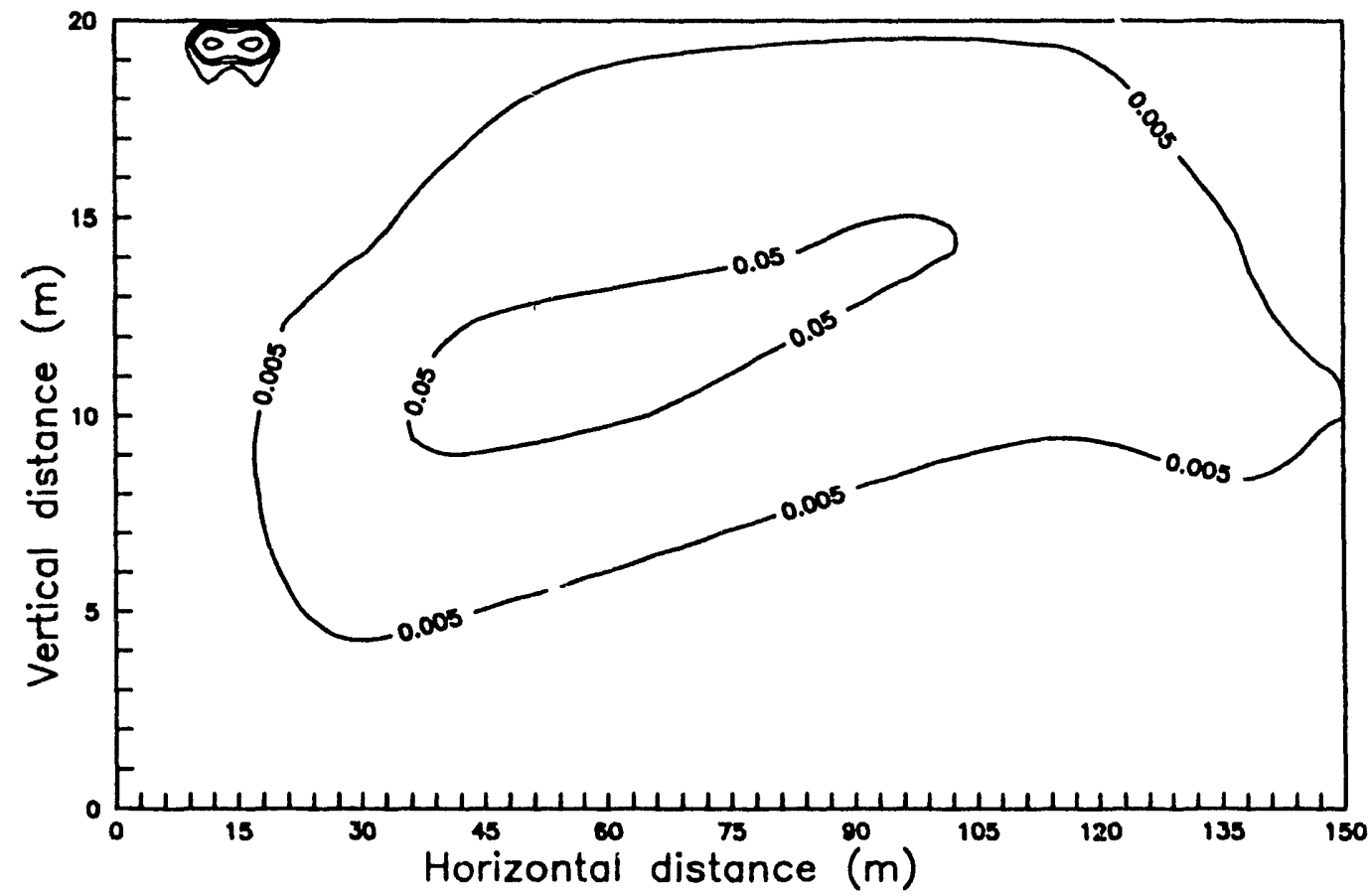

(b)

Figure 3.5. Concentration contours of conservative contaminant transport at $t$ $=150 \mathrm{y}$ with $\phi_{\mathrm{m}}=0.05$ as simulated by FRACTRAN (a) and VAM2D (b). 


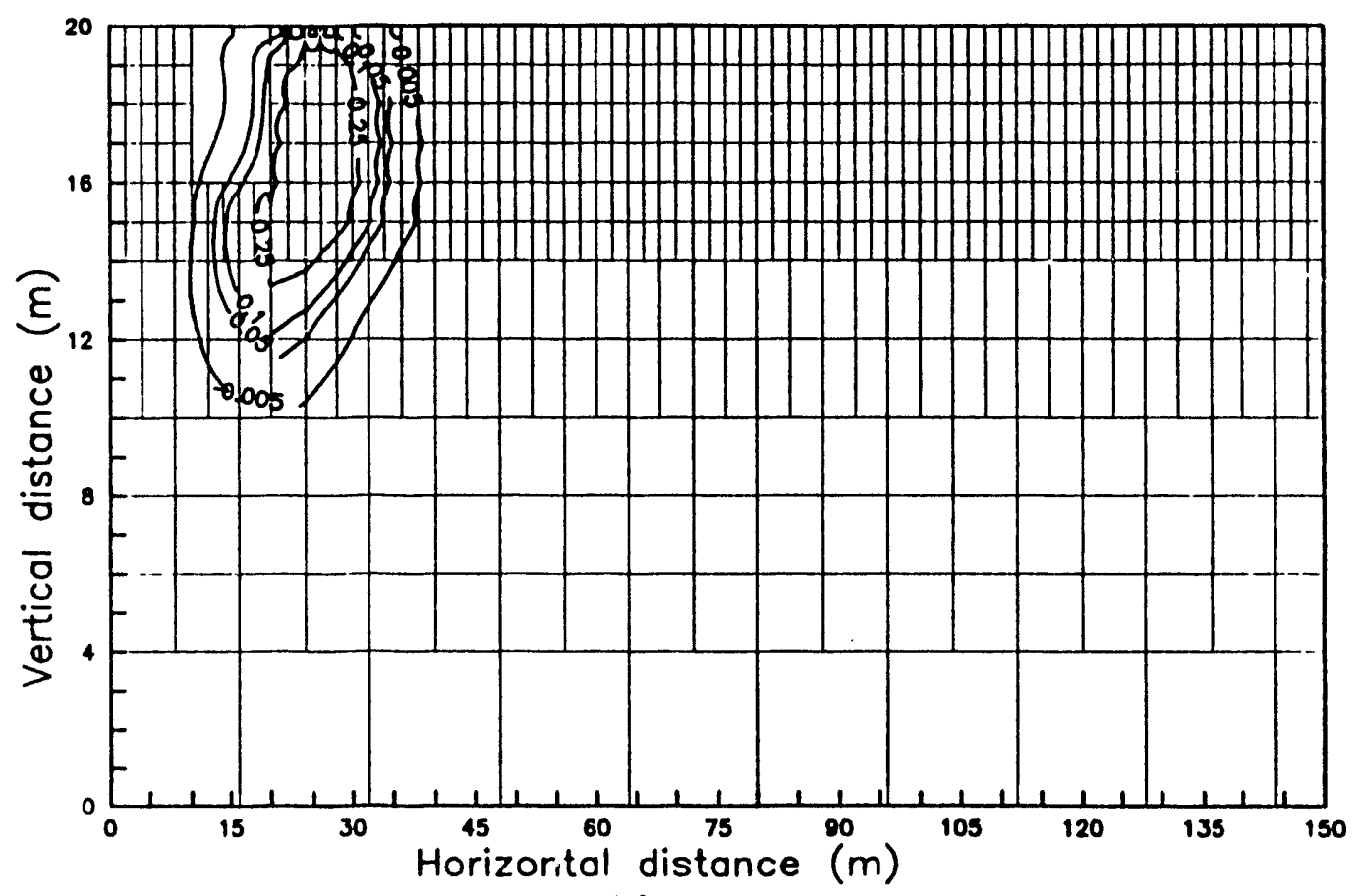

(a)

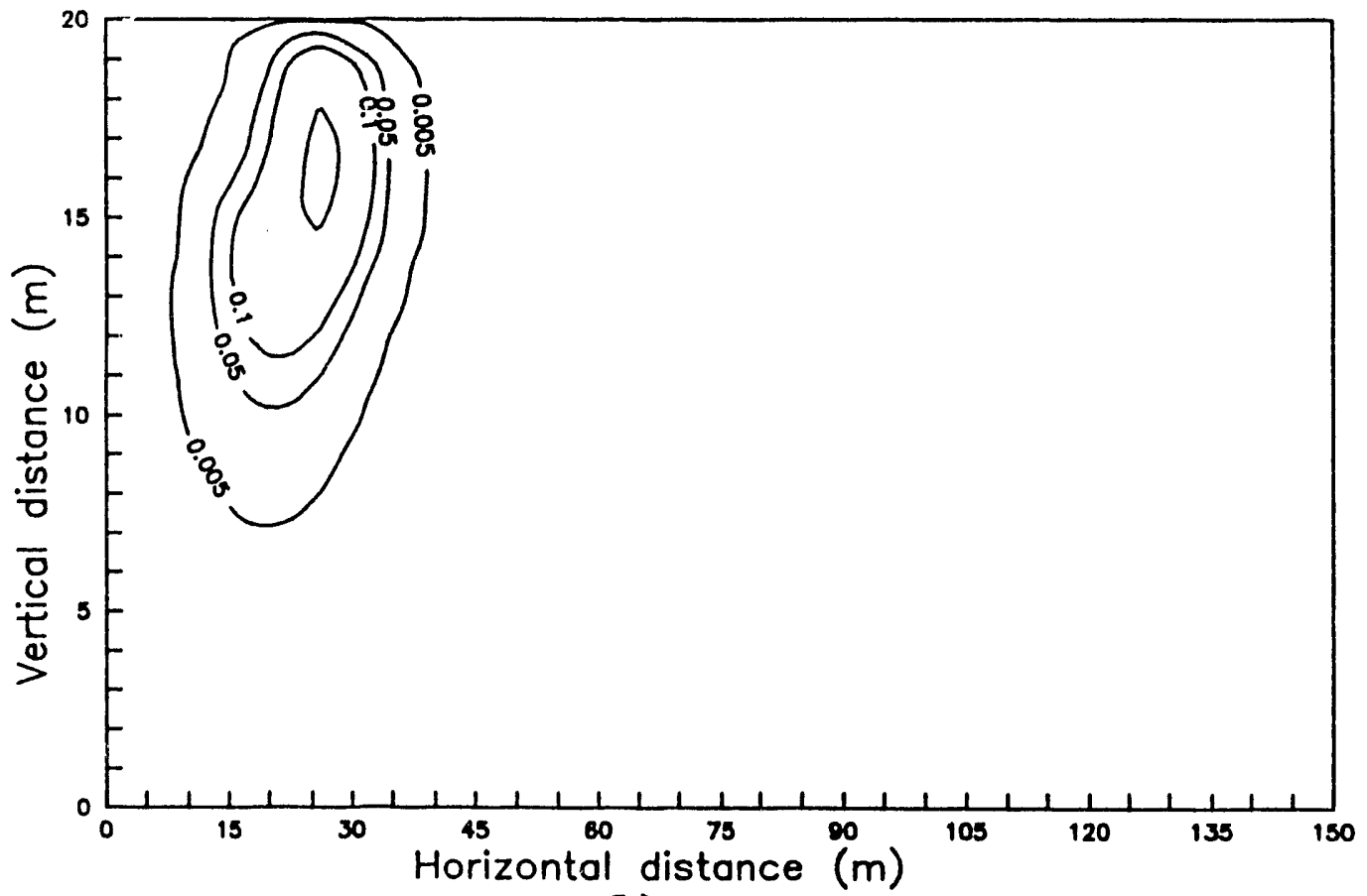

(b)

Figure 3.6. Concentration contours of conservative contaminant transport at $t$ $=50 \mathrm{y}$ with $\phi_{\mathrm{m}}=0.25$ as simuiated by FRACTRAN (a) and VAM2D (b). 


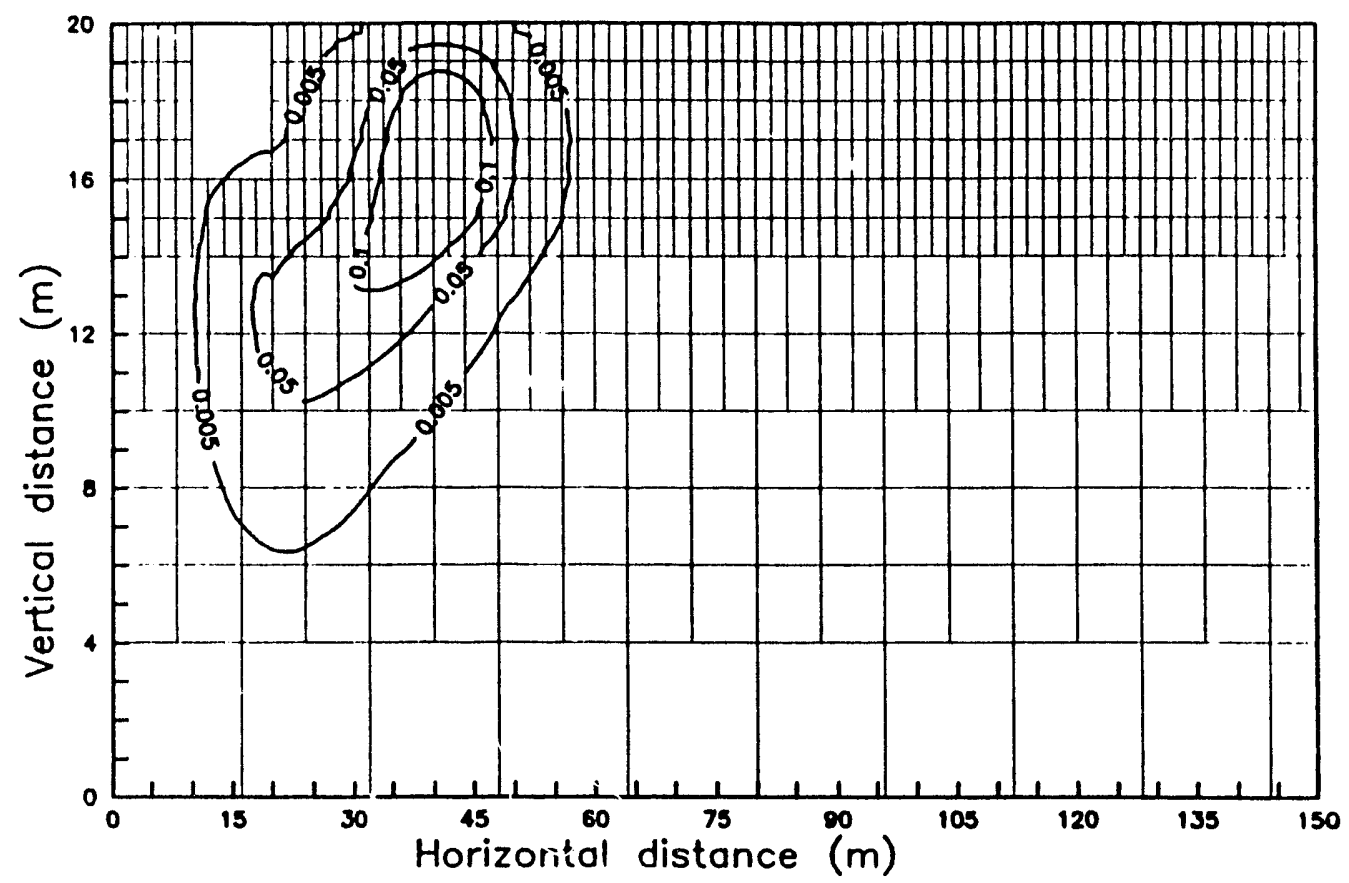

(a)

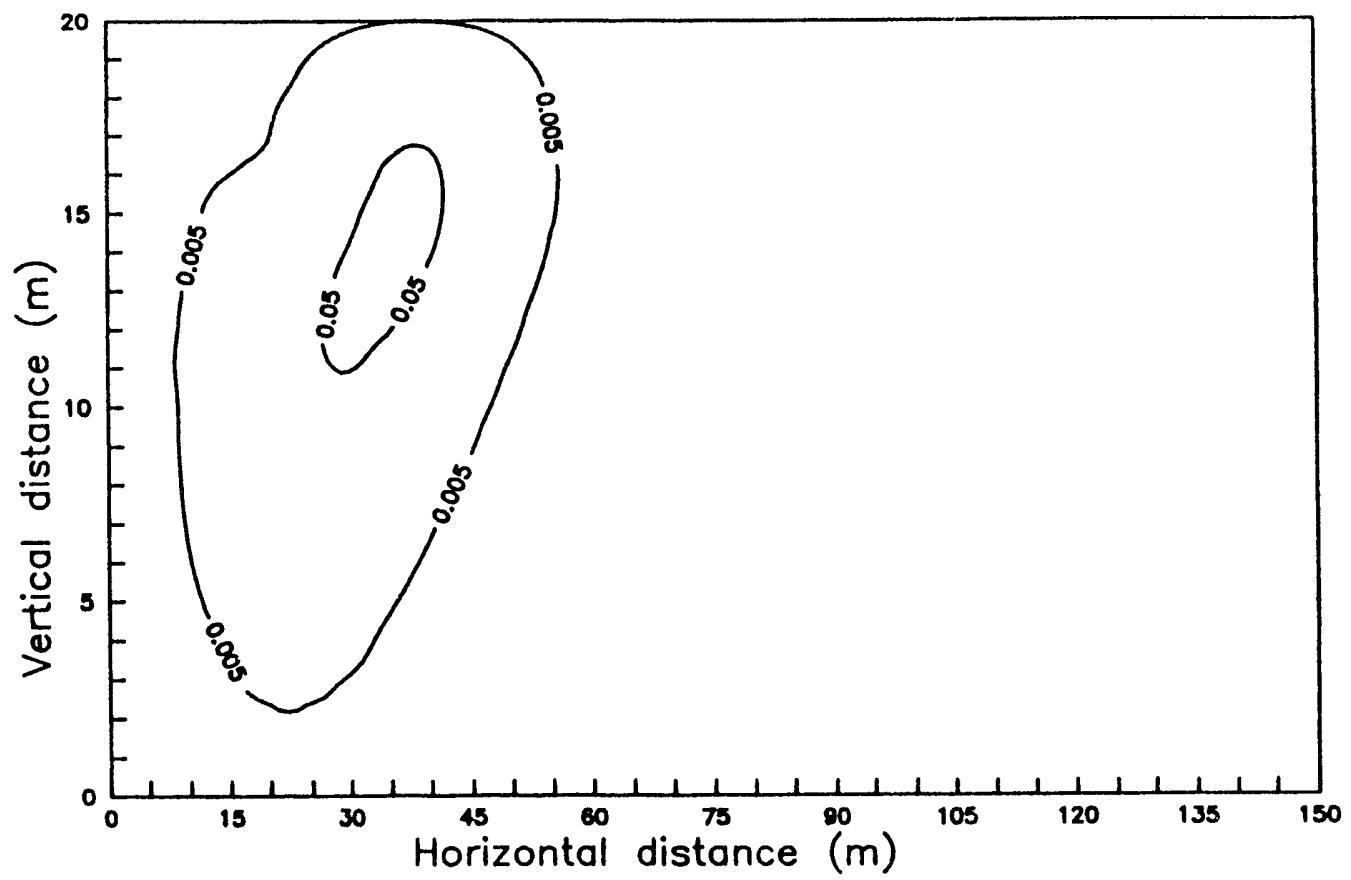

(b)

Figure 3.7. Concentration contours of conservative contaminant transport at $t$ $=150 \mathrm{y}$ with $\phi_{\mathrm{m}}=0.25$ as simulated by FRACTRAN (a) and VAM2D (b). 
a reduced seepage velocity and dispersivity. Due to the very small vertical flow component, downward plume movement is controlled by diffusion, which is enhanced compared to the low porosity case and thus results in greater downward plume movement. The vertical exaggeration in the figures should again be noted. The comparison batween VAM2D and the 'true' FRACTRAN results is quite favorable for this higher porosity case. The enhanced diffusion results in smoother FPACTRAN contours which improves agreement with the porous medium model. The extent of the contaminant plume, as indicated by the 0.005 relative concentration contour is predicted quite well by VAM2D both at $t=50$ years and at $t=150$ years. It can be seen that in this case, VAM2D tends to somewhat overpredict the downward extent of the plume. At $t=150$ years, VAM2D predicts that the plume limit will reached an elevation of $y=2 \mathrm{~m}$ above the lower model boundary, where as the FRACTRAN results indicate that the plume edge is at $y=6 \mathrm{~m}$. As in the first case, it can be seen again that, due to back dispersion across the top boundary, VAM2D tends to underpredict the plume peak concentrations, as well as the total amount of contaminant mass present in the plume. However, the boundary effect is less important than in the first case; while it affects the VAM2D predicted peak concentration level, it does not affect the prediction of the position of the peak.

\subsubsection{Reactive Contaminant Transport}

The third transport scenario considered is that of a contaminant which is subject to equilibrium sorption/desorption reactions. The sorption coefficients used are typical for metal ions, e.g. radionuclides. Radionuclide decay reactions are not considered. During initial simulations it was found that retardation factors were so high that, except for negligibly small decay rates, virtually all contaminant would be removed during time periods long enough to show significant plume migration. Another consideration is that radionuclide decay is completely independent of any fracture effects and is thus not directly relevant to the objectives of the present study. For the reactive contaminant modeling, the matrix porosity value of $\phi_{\mathrm{m}}$ $=0.05$ was used, since the contrast between fractures and matrix is more pronounced at low porosity values. To further emphasize fracture effects, the distribution coefficients were selected such that the retardation factor in the matrix would be significantly higher than the fracture value. For the porous matrix, a distribution coefficient value of $k_{d}=10^{-2} \mathrm{~m}^{3} / \mathrm{kg}$ was used. The corresponding retardation factor is then calculated via

$$
R_{m}=1+\frac{\rho_{b} k_{d}}{\phi_{m}}
$$

where $\rho_{\mathrm{b}}$ is the matrix bulk density, which in turn is estimated as:

$$
\rho_{b}=\left(1-\phi_{m}\right) \rho_{s}
$$

where a value of $2650 \mathrm{~kg} / \mathrm{m}^{3}$ was used for the solid densit. , $\rho_{\mathrm{s}}$. The res.lting value for $R_{\mathrm{m}}=$ 504.6. It can be clearly seen from this that a low porosity and corresponding high bulk density will result in a large matrix retardation factor. For fractures, the retardation factor is calculated as (Freeze and Cherry, 1979) 


$$
R_{f}=1+\frac{k_{a}}{b}
$$

where the fracture distribution coefficient, $k_{a}$, has dimensions of length. A value of $k_{a}=0.002$ $m$ was used. The corresponding value of the retardation factor $R_{f}$ depends on the fracture halfaperture $b$ and is thus different for each of the fracture zones. The values of $R_{f}$ are listed in Table 3.5. It can be seen that in the upper two fracture layers, through which most of the transport occurs, the value of $R_{f}$ is 5-8 times smaller than the matrix retardation factor. The effective retardation factor, $R_{e}$, used in the VAM2D simulations is calculated using (21). These values are also listed in Table 3.5, and illustrate that the effective retardation factor is determined mostly by the retardation in the matrix; the presence (or absence) of sorption in the fractures has little effect on overall retardation in the EPM approach.

FRACTRAN and VAM2D results for the reactive transport case are presented in Figures 3.8 and 3.9. Concentration contours after simulation times of $t=2,500$ years and $t=10,000$ years are shown. The large time values reflect the much slower contaminant migration rates compared to the two non-reactive cases. The different horizontal and vertical scale of Figures 3.8 and 3.9 compared to Figures 3.4 - 3.7 should also be noted. The selected retardation parameter values emphasize the contrast between matrix and fractures as can be seen in the jagged contours in the FRACTRAN plots, particularly at the earlier time value. The VAM2D results again show some anomalies near the contaminant source including a spurious secondary plume in the clay cover. The VAM2D simulation is also not able to reproduce the "fingering" of the concentration front along the fractures, resulting in some underprediction of the rate of migration of the contaminant front. Overall, however, the agreement of the VAM2D simulated plume and the 'true' FRACTRAN plume is very good. VAM2D predicts the position and overall shape of the contaminant plume quite well, even over the very long simulation times involved in this case. A comparison between Figures 3.8 and 3.9 on the one hand, and Figures 3.4-3.7 on the other hand suggests that the agreement between VAM2D and FRACTRAN for the reactive case is even better than it is for the two non-reactive cases considered. This is a favorable result since ficr many contaminants of practical concern, especially radionuclides, significant sorption effects will occur under many real world conditions. 


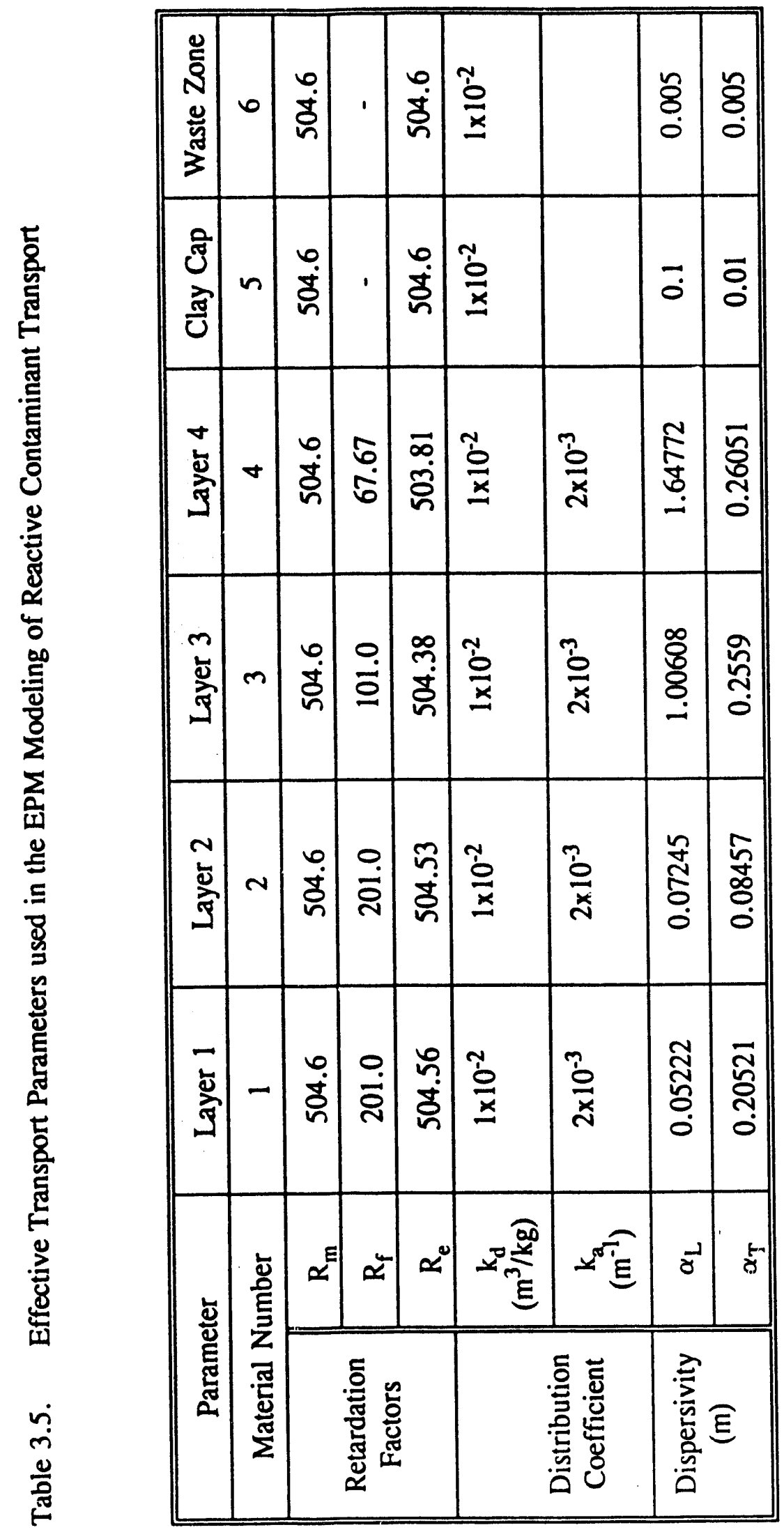




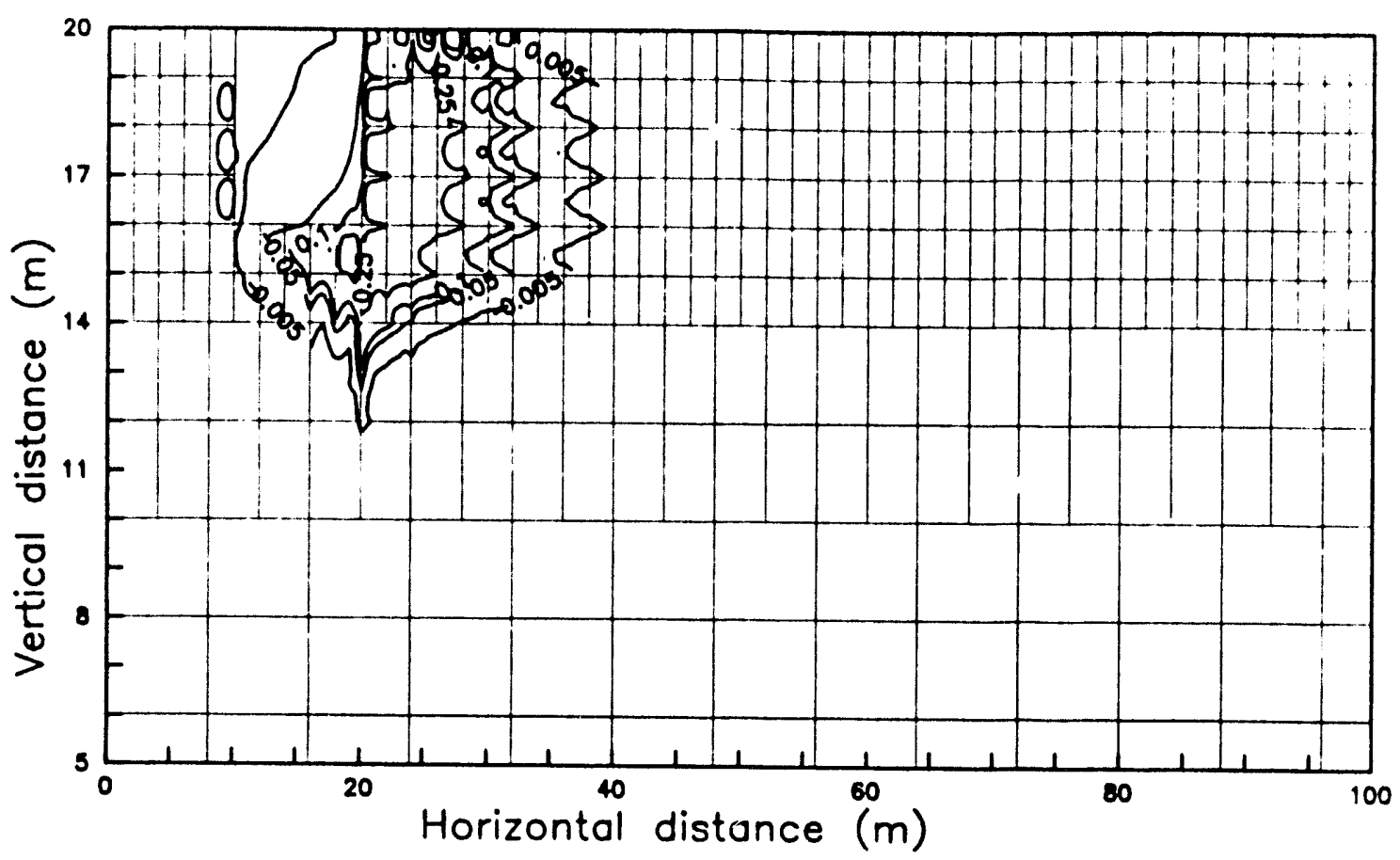

(a)

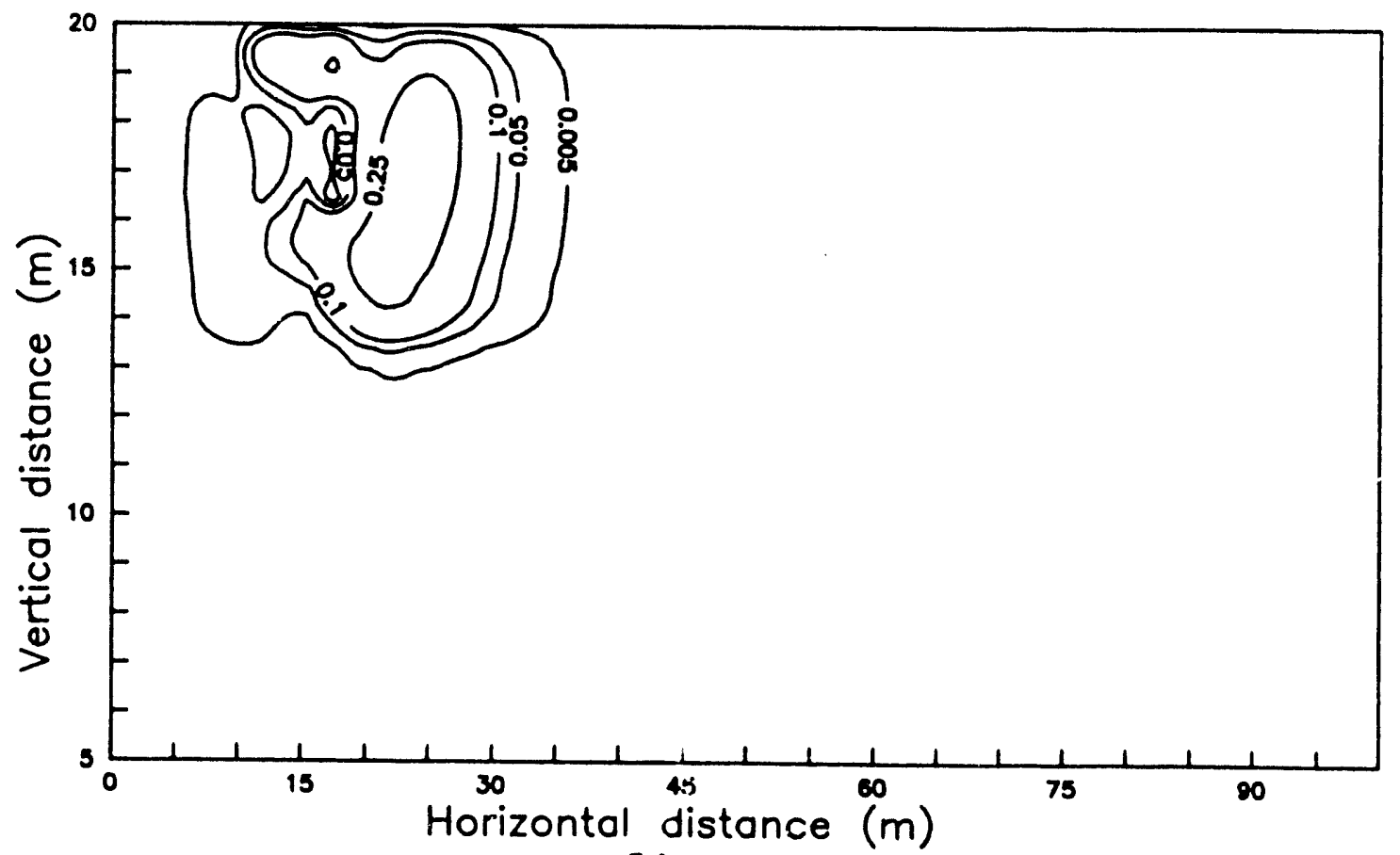

(b)

Figure 3.8. Concentration contours of reactive contaminant transport at $t=$ 2,500 y as simulated by FRACTRAN (a) and VAM2D (b). 


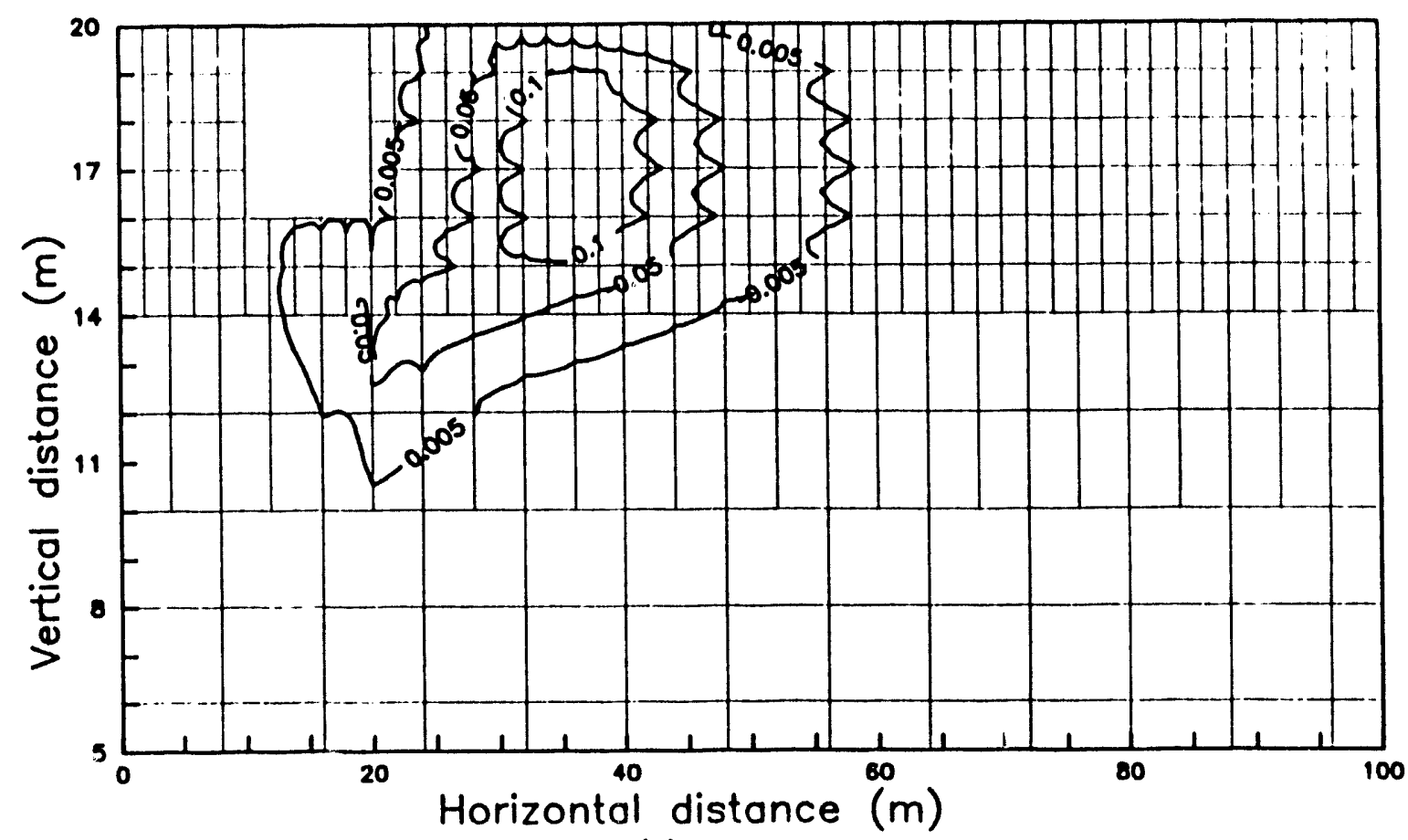

(a)

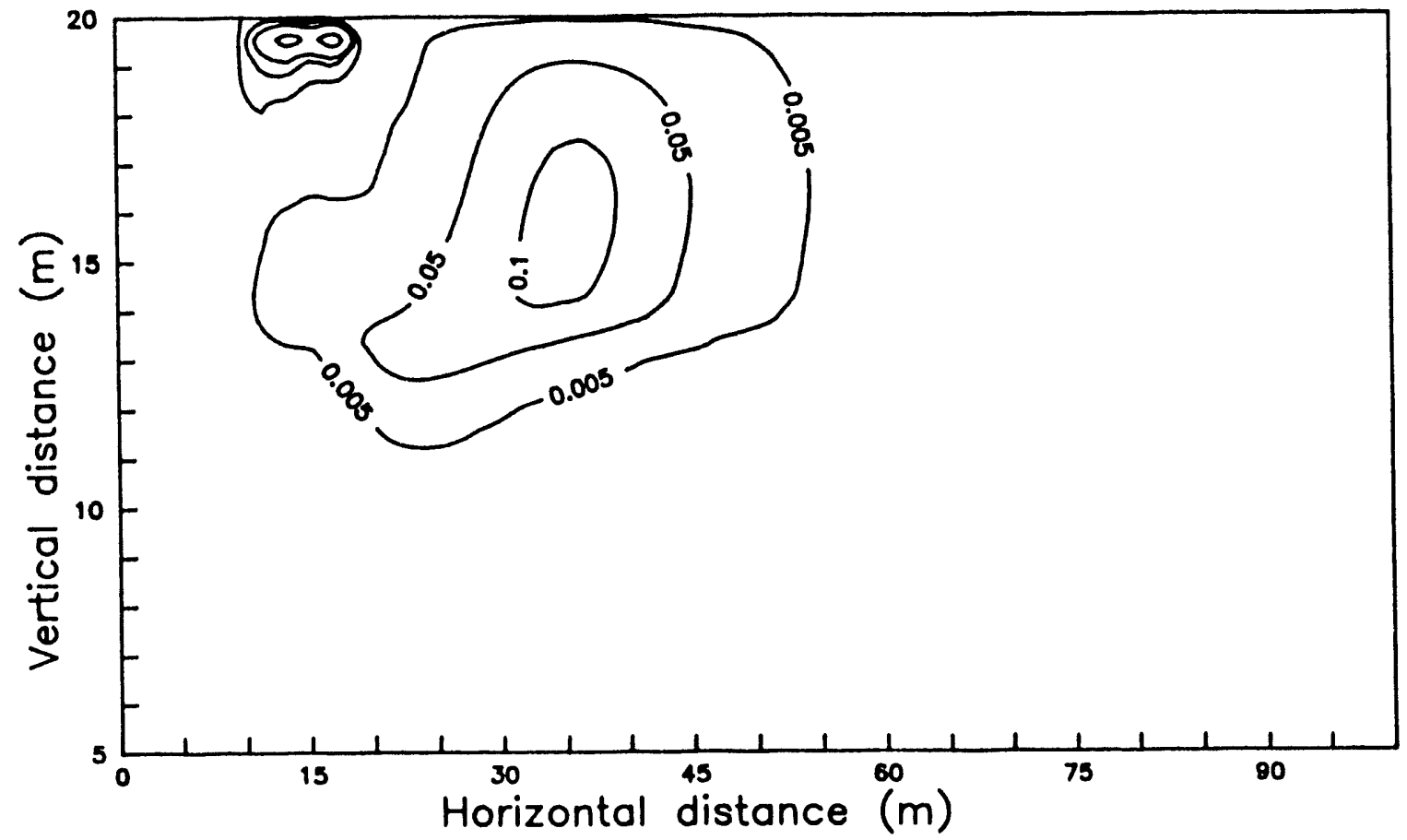

(b)

Figure 3.9. Concentration contours of reactive contaminant transport at $t=$ 10,000 y as simulated by FRACTRAN (a) and VAM2D (b). 


\section{DISCUSSION}

In this report we have presented relationships to compute effective flow and transport parameters in equivalent porous medium (EPM) modeling of fractured groundwater systems, and evaluated the predictive accuracy of these approximations for a hypothetical waste disposal site under a number of different transport conditions. Flow and transport modeling results obtained using the VAM2D porous medium model have been compared against simulations using the FRACTRAN discrete fracture floy and transport model. The results obtained using the latter model are taken to represent the 'true' behavior of the system. Steady state hydraulic head contours predicted by VAM2D agree closely with the FRACTRAN results, indicating that the EPM approach is quite effective in describing steady state groundwater flow under the conditions considered. Three different transport scenarios have been considered, involving different matrix porosities and conservative as well as reactive contaminants. Comparison of contaminant migration rates and plume shapes under the different conditions reveal the sensitivity to various transport parameters as well as provide some indication of the applicability of the EPM approach for transport modeling under different conditions.

In this study we have evaluated the use of predictive relationships to determine the flow and transport input parameters for the VAM2D porous medium model. This approach is in contrast to the method of estimating flow and transport parameters by fitting hydraulic and transport test data obtained, for instance, from pump tests and tracer tests. Effective flow and transport parameters used in the VAM2D modeling were successfully determined from assumed known fracture characteristics using relationships that are readily available from the literature. Existing expressions for the effective transport parameters pertain to conditions of steady state, unidirectional flow; our results suggest that these relationships can be successfully extended to flow in a multi-dimensional fracture network.

The transport conditions considered here involved different values of matrix porosity and sorbing versus non-sorbing contaminants. For a given Darcy flow rate, a lower porosity value will result in faster seepage velocity and therefore more rapid contaminant migration. A low porosity value in the porous matrix will also tend to reduce diffusional exchange between matrix and fractures and thus to more pronounced fracture effects on transport. The results presented here indicate that the effect of varying matrix porosity can be adequately accounted for in an EPM approximation of contaminant transport. Incorporation of sorption reactions into the transport modeling can very drastically change the time scale required for contaminant migration. Retardation factors considered in the third transport modeling scenario are rather high, but by no means exceptional for many radionuclides. VAM2D predictions of contaminant migration for the sorbing case involving large simulation times were at least as accurate as those for the non-reactive simulations, indicating that linear equilibrium sorption reactions with different matrix and fracture retardation factors can be effectively accounted for in the EPM approach. An unexpected result which showed up in some of the simulations is the significant dispersive mass loss across the model top boundary in the VAM2D simulations. This effect is especially clear in the 150 year result for the low matrix porosity simulation. This boundary effect is related to the use of a first-type boundary condition along the upper boundary of the 
modeled region and could easily be removed by simply moving the boundary away from the location of the plume. On the other hand, this back dispersion effect also illustrates the spurious results that may arise from the use of large dispersivity values in an EPM approximation of transport in fractured media, and of which a modeler should consequently be aware.

The results of this investigation are, on the whole, quite favorable with regard to the applicability of the EPM approach for modeling flow and transport in a fractured groundwater system, and with regard to the possibility of determining effective flow and transport parameters from known fracture and matrix characteristics. Nevertheless, it must also be recognized that the present study constitutes only a limited validation of the EPM modeling approach. Two main considerations may limit the applicability of this study to real world conditions. The first is related to the relative simplicity of the fracture network at the hypothetical waste disposal site; the second is related to the accuracy with which the fracture characteristics are known. We have considered a two-dimensional, exactly orthogonal network of interconnected, constant aperture, planar fractures, embedded in a uniform porous matrix. In reality, a great deal more variation can be expected in fracture shape, spacing, orientation, aperture and degree of fracture connectivity. Various researchers have studied the effect of factors related to fracture geometry on flow and transport behavior of fractured media (e.g. Smith and Schwartz, 1984; Shimeo and Long, 1987). In general, such studies have shown that flow and transport behavior will deviate from porous media-like behavior as the fracture network becomes more irregular, i.e., exhibits more variation in fracture apertures, fewer interconnected fractures, etc. Based on conclusions from these studies, the regular fracture network assumed in our investigation favors the EPM approach. It is less clear however, to what extent complex fracture geometries limit the applicability of the EPM approach in natural geologic media. Most modeling studies of complex fracture geometries ignore the effect of matrix diffusion on transport and instead use channeling models for transport in which fractures are the only conduits for fluid and solutes. Ignoring the averaging effects of matrix diffusion may very well lead to overly pessimistic conclusions regarding the applicability of continuum approaches for fracture networks of realistic complexity.

In determining the effective flow and transport parameters for the VAM2D simulations we assumed that the fracture characteristics were known exactly. In a real world situation it will be very rare that such accurate and detailed data are available. In practice, information on fracture spacing and orientation may be obtained by mapping fractures along exposed faces or boreholes. Accurate direct measurements of fracture connectivity and aperture, however, will be very difficult to obtain. The only viable alternative then may be to employ inverse procedures to estimate or back-calculate the needed model parameters from flow and transport data. The latter approach will often require expensive and/or time consuming field tests.

The mentioned limitations of our study not withstanding, we feel our results do support the notion that porous medium flow and transport models such as VAM2D can be used to simulate flow and transport in fractured groundwater systems under conditions similar to those of our study. In applying models to predict flow and transport behavior at actual field sites, the lack of accurate values for model input parameters is in many cases a major factor controlling the ultimate reliability of modeling results. Since physically more rigorous (and complex) 
models will generally require more data for calibration that do simpler models, it may be well justified to limit modeling efforts to a traditional porous medium representation. We have shown that information on fracture characteristics, some of which may be obtained from visual observations, can be readily incorporated into the porous medium approximations. Although there is a great need for field studies to validate the various approaches for modeling fractured media, there: may be many cases in which the accuracy of the EPM approach is commensurate with available data as well as with the attainable level of accuracy and reliability of model predictions. 


\section{REFERENCES}

\section{PART 1 APPLICATION OF THE VAM2D CODE TO THE SECOND LAS CRUCES TRENCH EXPERIMENT}

Celia, M.A., and E.T. Bouloutas, and R.L. Zarba, 1990. A general mass-conservative numerical solution for the unsaturated flow equation. Wat. Resour. Res., 26: 14831496.

Collin, M., M. Lindgren, and A. Rasmuson, 1990. Flow and transport simulations of the second Las Cruces trench experiment, INTRAVAL Case 10, Report No. KEMAKTA AR 90-04, KEMAKTA Consultants Co., Sweden (DRAFT).

Cooley, R.L., 1983. Some new procedures for numerical solution of variably saturated flow problems, Wat. Resour. Res., 19: 1271-1285.

Dagan, G., 1989. Flow and transport in porous formations. Springer-Verlag, Berlin Heidelberg, $465 \mathrm{pp}$.

Freyberg, D.L., 1986. A natural gradient experiment on solute transport in a sand aquifer 2. Spatial moments and the advection and dispersion of non-reactive tracers. Wat. Resour.

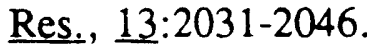

Goodrich, M.T., and P.A. Davis, 1989. A statistical analysis of the Las Cruces trench hydraulic data. INTRAVAL workshop, Helsinki, Finland.

Hills, R.G., I. Porro, D.B. Hutson, and P.J. Wierenga, 1989. Modeling one-dimensional infiltration into very dry soils, 1. Model development and evaluation. Wat. Resour. Res., 25: 1259-1269.

Hornung, U., and Messing, W., 1984. Poröse Medien-Methoden und Simulation. Verlag Beiträge zur Hydrologie, Kirchzarten, F.R.G.

Huyakorn, P.S., J.B. Kool, and J.B. Robertson, May 1989. VAM2D - Variably saturated analysis model in two dimensions. Report No. NUREG/CR-5352, U.S. Nuclear Regulatory Commission, Washington, D.C.

Huyakorn, P.S., J.W. Mercer, and D.S. Ward, 1985. Finite element matrix and mass balance computational schemes for transport in variably saturated porous media, Wat. Resour. Res., 21: 346-358. 
Huyakom, P.S., S.D. Thomas, and B.M. Thompson, 1984. Techniques for making finite elements competitive in modeling flow in variably saturated porous media, Wat. Resour. Res., 20: 1099-1115.

Huyakorn, P.S., S.D. Thomas, and B.M. Thompson, 1984. Techniques for making finite elements competitive in modeling flow in variably saturated porous media. Wat. Resour. Res., 20: 1099-1115.

Huyakorn, P.S., and G.F. Pinder, 1983. Computational Methods in Subsurface Flow, Academic Press, Orlando, Florida, 474 pp.

Kool, J.B., and J.C. Parker, 1987. Development and evaluation of closed-form expressions for hysteretic soil hydraulic properties, Wat, Resour. Res., 23: 105-114.

McCord, J.T., D.B. Stephens, and J.W. Wilson, 1988. Field experiments and numerical simulations of unsaturated flow and transport: The roles of hysteresis and statedependent anisotropy. Proceedings Nato Advanced Study Institute on Recent Advances in Modeling Hydrologic Systems, Sintra, Portugal, July 9-23.

Millington, R.J., and J.M. Quirk, 1961. Permeability of porous solids. Trans. Faraday Society, 57: 1200-1207.

Milly, P.C.D., 1985. A mass-conservative procedure for time-stepping in models of unsaturated flow. Adv, Wat. Resour., 8:32-36.

Peck, A.J., 1983. Field variability of soil physical properties. In: Advances in Irrigation, Vol. 2. Academic Press, Inc.

Polmann, D.M., E.G. Vomvoris, D. Mclaughlin, E.M. Hammick, and L.W. Gelhar, September, 1988. Application of stochastic methods to the simulation of large-scale unsaturated flow and transport. Report No. NUREG/CR-5094, U.S. Nuclear Regulatory Commission, Washington, D.C.

Robertson, J.B., 1984. Geologic problems at low-level radioactive waste sites; in: Groundwater Contamination, National Research Council, National Academy Press, p. 104-109.

Russo, D., and G. Dagan, 1991. On solute transport in heterogeneous porous formation under saturated and unsaturated water flows. Wat. Resour, Res., 27:285-292.

Shipers, L.R., and C.P. Harlan, December, 1988. Background information for the development of a low-level waste performance assessment methodology. Volume 2: Assessment of relative significance of migration and exposure pathways. Report No. NUREG/CR5453, U.S. Nuclear Regulatory Commission, Washington, D.C. 
Sudicky, E.A., 1986. A natural gradient experiment on solute transport in a sand aquifer: Spatial variability of hydraulic conductivity and its role in the dispersion process. Wat. Resour. Res., 22:2069-2082.

van Genuchten, M. Th., 1982. A numerical model for water and solute movement in and below the rootzone. Research Report No. 121, USDA-ARS, U.S. Salinity Laboratory, Riverside, CA (DRAFT).

van Genuchten, 1980. A closed-form equation for predicting the hydraulic conductivity of unsaturated soils. Soil Sci. Soc. Am. J., 44:892-898.

Wierenga, P.J., D.B. Hudson, R.G. Hills, I. Porro, M.R. Kirkland, and J. Vinson, August, 1990. Flow and transport at the Las Cruces trench site, Experiments 1 and 2. Report No. NUREG/CR-5607, U.S. Nuclear Regulatory Commission, Washington, D.C.

Wierenga, P.J., A.F. Toormann, D.B. Hudson, J. Vinson, M. Nash, and R.G. Hills, November, 1989. Soil Physical properties at the Las Cruces trench site, Report No. NUREG/CR-5441, U.S. Nuclear Regulatory Commission, Washington, D.C.

Wierenga, P.J., 1988. Validation of flow and transport models at the Jornada test facility. In: P.J. Wierenga \& D. Bachelet (Eds.), Validation of Flow and Transport Models for the Unsaturated Zone: Conference Proceedings. Research Report 88-55-04, New Mexico State University.

Wierenga, P.J., L.W. Gelhar, C.S. Simmons, G.W. Gee, and T.J. Nicholson, August, 1986. Validation of stochastic flow and transport models for unsaturated soils: A comprehensive field study. Report No. NUREG/CR-4622, U.S. Nuclear Regulatory Commission, Washington, D.C.

Yeh, T.-C. J., L.W. Gelhar, and A.L. Gutjahr, 1985. Stochastic analysis of unsaturated flow in heterogenous soils 2. Statistically anisotropic media with variable $\alpha$. Wat. Resour. Res., 21:457-464.

PART 2 APPLICATION OF THE VAM2D COMPUTER CODE FOR MODELING OF FLOW AND TRANSPORT IN FRACTURED MEDIA USING AN EQUIVALENT POROUS MEDIUM APPROACH

Bear J., "On the Tensor Form of Dispersion in Porous Media," L. Seophysical Res., Vol. 66(14), pp. 1185-1197, 1961.

Bear, J., Dynamics of Fluids in Porous Media, American Elsevier, New York, NY, 1972. 
Berkowitz, B., J. Bear and C. Braester, "Continuum Models for Contaminant Transport in Fractured Porous Formations," Wat. Resour. Res, 24: 1225-1236, 1988.

Cherry, J.A., "Hydrogeologic Contaminant Behaviour in Fractured and Unfractured Clayey Deposits in Canada," Proceedings Intl. Conference on Contaminant Transport in Groundwater, Shuttgart, Germany, April, 1989.

de Marsily, G., Quantitative Hydrogeology, Groundwater Hydrology for Engineers, Academic Press, San Diego, CA, 1986.

Dullien, F.A.L., Porous Media Fluid Transport and Pore Structure, Academic Press, New York, N.Y., 1979.

Endo, H.K., "Mechanical Transport in Two-Dimensional Network of Fractures," Ph.D. Thesis, LBL-17491, Earth Sciences Division, Lawrence Berkeley Laboratory, University of California, Berkeley, CA, 1984.

Freeze, R.A. and J.A. Cherry, Groundwater, Prentice Kall, Inc., Englewood Cliffs, N.J., 1979.

Fried, J.J., Groundwater Pollution, Elsevier Scientific Publishing Co., New York, NY 10017, 1975.

Grisak, G.E. and J.F. Pickens, "Solute Transport Through Fractured Media 2. Column Study of Fractured Till," Wat. Resour, Res., 16: 731-739, $1980 \mathrm{~b}$.

Grisak, G.E. and J.A. Cherry, "Hydrologic Characteristics and Response of Fractured Till and Clay Confining a Shallow Aquifer," Canadian Geotechnical Journal, 12: 23-43, 1975.

Grisak, G.E. and J.F. Pickens, "Solute Transport Through Fractured Media 1. The Effect of Matrix Diffusion," Wat. Resour, Res, 16: 719-730, 1980a.

Huyakorn, P.S., J.B. Kool and J.B. Robertson, VAM2D - Variably Saturated Analysis Model in Two Dimensions, Version 5.0, NUREG/CR-5352, U.S. Nuclear Regulatory Commission, Washington, D.C., 1989.

Khaleel, R., "Scale Dependence of Continuum Models for Fractured Basalts," Wat. Resour. Res., 25: 1847-1855, 1989.

Long, J.C.S., J.S. Remer, C.R. Wilson and P.A. Witherspoon, "Porous Media Equivalents for Networks of Discontinuous Fractures," Wat. Resour. Res., 18: 645-658, 1982.

Maini, T. and G. Hocking, "An Examination of the Feasibility of Hydrologic Isolation of a HIgh Level Waste Repository in Crystalline Rocks," Annual Meeting Geological Society of America, Seattle, WA, 1977. 
Neretnieks, I., "Diffusion in the rock Matrix, An important factor in radionıclide retardation," Lournal of Geophysical Research, 85: 4379-4397, 1980.

Pankow, J.F., R.L. Johnson, J.P. Newetson and J.A. Cherry, "An Evaluation of Contaminant Migration Patterns at two waste disposal Sites on Fractured Porous Media in Terms of the Equivalent Porous Medium (EPM) model, " Journal of Contaminant Hydrology, 1 : 65-76, 1986.

Parker, J.C. and A.J. Valocchi, "Constraints on the Validity of Equilibrium and First-Order Kinetic Transport Models in Structured Soils," Wat. Resour, Res., 22: 399-407, 1986.

Parker, J.C., and M.Th. van Genuchten, "Determining Transport Parameters From Laboratory and Field Tracer Experiments," VAES Bulletin, No. 84-3, Virginia Polytechnic Institute and State University, Blacksburg, VA 24061, 1984.

Scheidegger, A.E., "General Theory of Dispersion in Porous Media," L. Geophysical Res., 66: 3273-3278, 1961.

Shimo, M. and J.C.S. Long, A Numerical Study of Transport Parameters in Fracture Networks, in D.D. Evans and T.J. Nicholson (Eds.), Flow and Transport Through Unsaturated Fractured Rock, pp. 121-131, Geophysical Monograph 42, Americal Geophysical Union, Washington, DC, 1987.

Snow, D.T., "Anisotropic Permeability of Fractured Media," Wat. Resourc. Res., 5: 1273$1289,1969$.

Sudicky, E.A., "The Laplace Transform Galerkin Technique: A Time-Continuous Finite Element Theory and Application to Mass Transport in Groundwater," Wat. Resour. Res., 25: 1833-1846, 1989a.

Sudicky, E.A., "The Laplace Transform Galerkin Technique for Large-Scale Simulation of Mass Transport in Discretely Fractured Porous Media", EOS Transaction American Geophysical Union, 70: 1082, $1989 \mathrm{~b}$.

Tang, D.H., E.O. Frind, and E.A. Sudicky, "Contaminant Transport in Fractured Porous Media: Analytical Solution For a Single Fracture," Wat. Resour. Res. 17: 555-564, 1981.

Taylor, G.E., "Dispersion of Soluble Matter in Solvent Flowing Slowly Through a Tube," Proceedings Royal Society of London. Series A, 1137: 186-203, 1953.

van Genuchten, M. Th. and F.N. Dalton, "Models for Simulating Salt Movement in Aggregated Field Soils," Geoderma, 1387: 165-183, 1986. 
van Genuchten, M.Th., "A General Approach for Modeling Solute Transport in Structured Soils", Memoires IAHS, 17: 513-526, 1985.

van Genuchten, M. Th. and W.A. Jury, "Progress in Unsaturated Flow and Transport Modeling", Contributions in Hydrology, U.S. National Report to International Union of Geology and Geophysics, 1983-1986, pp. 135-140, 1987.

Vorauer, A.G., D.W. Hardin', M.B. Dusseault and J.A. Cherry, "The Nature of Near-Surface Fractures in Clay Tills of Southwestern Ontario", Proceedings 3rd Hydrogeological Conference, IAHS, Canadian Chapter, Saskatoon, Saskatchewan, April 21-23, 1986. 


\section{APPENDIX \\ Values of van Genuchten Soil Parameters for Individual \\ Soil Cores Used in the \\ VAM2D Simulations}


This Appendix contains the values of the van Genuchten hydraulic parameters for each of the individual soil cores collected from the trench. The values that are given here are the ones used in simulation scenarios IIa-c and III. These values are different from the ones given in the Las Cruces trench database since an upper limit was set for the parameter $\theta_{\mathrm{r}}$ (WCR) in the fitting procedure, based on the minimum observed water content for each soil layer in the field. Imposing constraints on $\theta_{\mathrm{r}}$ affects the fitted values of parameters $\alpha$ and $\beta$ also. The following information is provided:

Sample ID number

$x$-coordinate of sample location $(m)$

$y$-coordinate of sample location $(m)$

$\mathrm{z}$-coordinate of sample location $(\mathrm{m})$

Saturated water content

Residual water content

Parameter $\alpha\left(\mathrm{cm}^{-1}\right)$

Parameter $\beta$

\author{
SAMPLE \\ $x$ \\ y \\ $\mathrm{y}$ \\ WCS \\ WCR \\ ALPHA \\ BETA
}

The sample ID number is the same as used in the Las Cruces trench database (Wierenga et al., 1989). The sample location is given using the convention used throughout this report; the origin for the $x$-coordinate axis is the center of irrigation plot $\# 2$, the origin for the $y$-axis trench face with positive $y$-values away from the trench, and the origin for the $\mathrm{z}$-axis is at $5 \mathrm{~m}$ below the soil surface, with positive $z$-values going upward. The y-coordinate for all samples was constant, i.e. all samples were collected from transects parallel to the long axis of the trench and inside the trench at a distance of $60 \mathrm{~cm}$ from the trench face $(y=-0.6 \mathrm{~m})$. Missing parameters are assigned a numerical value of -999 . 


\begin{tabular}{|c|c|c|c|c|c|c|c|}
\hline SAMPLE & $x$ & $\mathbf{Y}$ & 2 & WCS & WCR & ALPHA & BETA \\
\hline $\begin{array}{l}1-1 \\
1-2 \\
1-3 \\
1-4 \\
1-5 \\
1-6 \\
1-7 \\
1-8 \\
1-9 \\
1-10 \\
1-11 \\
1-12 \\
1-13 \\
1-14 \\
1-15 \\
1-16 \\
1-17 \\
1-18 \\
1-19 \\
1-20 \\
1-21 \\
1-22 \\
1-23 \\
1-24 \\
1-25 \\
1-26 \\
1-27 \\
1-28 \\
1-29 \\
1-30 \\
1-31 \\
1-32 \\
1-33 \\
1-34 \\
1-35 \\
1-36 \\
1-37 \\
1-38 \\
1-39 \\
1-40 \\
1-41 \\
1-42 \\
1-43 \\
1-44 \\
1-45 \\
1-46 \\
1-47 \\
1-48 \\
1-49 \\
1-50 \\
2-1 \\
2-3 \\
2-5 \\
2-7\end{array}$ & $\begin{array}{r}-3.78 \\
-3.28 \\
-2.78 \\
-2.28 \\
-1.78 \\
-1.28 \\
-0.78 \\
-0.28 \\
0.22 \\
0.72 \\
1.22 \\
1.72 \\
2.22 \\
2.72 \\
3.22 \\
3.72 \\
4.22 \\
4.72 \\
5.22 \\
5.72 \\
6.22 \\
6.72 \\
7.22 \\
7.72 \\
8.22 \\
8.72 \\
9.22 \\
9.72 \\
10.22 \\
10.72 \\
11.22 \\
11.72 \\
12.22 \\
12.72 \\
13.22 \\
13.72 \\
-14.22 \\
-1.28 \\
-0.78\end{array}$ & $\begin{array}{l}-0.60 \\
-0.60 \\
-0.60 \\
-0.60 \\
-0.60 \\
-0.60 \\
-0.60 \\
-0.60 \\
-0.60 \\
-0.60 \\
-0.60 \\
-0.60 \\
-0.60 \\
-0.60 \\
-0.60 \\
-0.60 \\
-0.60 \\
-0.60 \\
-0.60 \\
-0.60 \\
-0.60 \\
-0.60 \\
-0.60 \\
-0.60 \\
-0.60 \\
-0.60 \\
-0.60 \\
-0.60 \\
-0.60 \\
-0.60 \\
-0.60 \\
-0.60 \\
-0\end{array}$ & $\begin{array}{l}4.94 \\
4.94 \\
4.94 \\
4.94 \\
4.94 \\
4.94 \\
4.94 \\
4.94 \\
4.94 \\
4.94 \\
4.94 \\
4.94 \\
4.94 \\
4.94 \\
4.94 \\
4.94 \\
4.94 \\
4.94 \\
4.94 \\
4.94 \\
4.94 \\
4.94 \\
4.94 \\
4.94 \\
4.94 \\
4.94 \\
4.94 \\
4.94 \\
4.94 \\
4.94 \\
4.94 \\
4.94 \\
4.94 \\
4.94 \\
4.94 \\
4.94 \\
4.94 \\
4.94 \\
4.94 \\
4.94 \\
4.94 \\
4.94 \\
4.94 \\
4.94 \\
4.94 \\
4.94 \\
4.94 \\
4.94 \\
4.94 \\
4.94 \\
4.29 \\
4.29 \\
4.29 \\
4.29 \\
4.29 \\
4.29 \\
4.29\end{array}$ & $\begin{array}{l}0.345 \\
0.352 \\
0.323 \\
0.311 \\
0.329 \\
0.301 \\
0.288 \\
0.300 \\
0.315 \\
0.381 \\
0.335 \\
0.345 \\
0.364 \\
0.343 \\
0.342 \\
0.309 \\
0.318 \\
0.333 \\
0.347 \\
0.341 \\
0.341 \\
0.384 \\
0.343 \\
0.364 \\
0.351 \\
0.342 \\
0.312 \\
0.364 \\
0.360 \\
0.334 \\
0.322 \\
0.376 \\
0.320 \\
0.335 \\
0.327 \\
0.339 \\
0.334 \\
0.336 \\
0.341 \\
0.369 \\
0.397 \\
0.373 \\
0.357 \\
0.342 \\
0.381 \\
0.359 \\
0.332 \\
0.338 \\
0.342 \\
0.366 \\
0.285 \\
0.277 \\
0.291 \\
0.270 \\
0.312 \\
0.293 \\
0.286\end{array}$ & $\begin{array}{l}0.0350 \\
0.0350 \\
0.0350 \\
0.0350 \\
0.0350 \\
0.0350 \\
0.0350 \\
0.0350 \\
0.0350 \\
0.0350 \\
0.0350 \\
0.0350 \\
0.0350 \\
0.0350 \\
0.0350 \\
0.0350 \\
0.0250 \\
0.0350 \\
0.0350 \\
0.0350 \\
0.0350 \\
0.0350 \\
0.0350 \\
0.0350 \\
0.0350 \\
0.0350 \\
0.0350 \\
0.0350 \\
0.0350 \\
0.0350 \\
0.0350 \\
0.0350 \\
0.0350 \\
0.0350 \\
0.0350 \\
0.0350 \\
0.0350 \\
0.0350 \\
0.0350 \\
0.0350 \\
0.0350 \\
0.0350 \\
0.0350 \\
0.0350 \\
0.0350 \\
0.0350 \\
0.0350 \\
0.0350 \\
0.0350 \\
0.0350 \\
0.0350 \\
0.0350 \\
0.0350 \\
0.0350 \\
0.0350 \\
0.0350\end{array}$ & 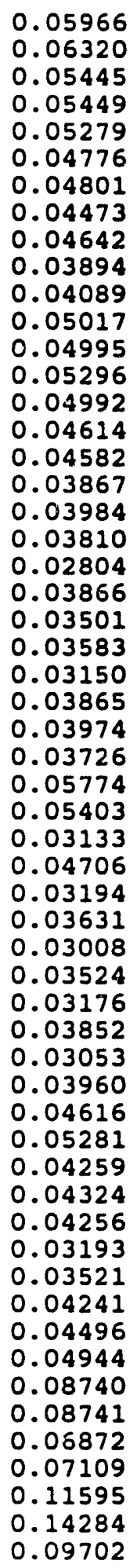 & $\begin{array}{l}1.5512 \\
1.5273 \\
1.4862 \\
1.5824 \\
1.4984 \\
1.6520 \\
1.5453 \\
1.6543 \\
1.5491 \\
1.8007 \\
1.5313 \\
1.5496 \\
1.4942 \\
1.5021 \\
1.6663 \\
1.5663 \\
1.4875 \\
1.5751 \\
1.6613 \\
1.5817 \\
1.6524 \\
1.6471 \\
1.5037 \\
1.5236 \\
1.5693 \\
1.4851 \\
1.4770 \\
1.5925 \\
1.5650 \\
1.4410 \\
1.4715 \\
1.5334 \\
1.4852 \\
1.5652 \\
1.6256 \\
1.6003 \\
1.5801 \\
1.4842 \\
1.5847 \\
1.5264 \\
1.5933 \\
1.5808 \\
1.5727 \\
1.5082 \\
1.5934 \\
1.6296 \\
1.5424 \\
1.4720 \\
1.5219 \\
1.5214 \\
1.3351 \\
1.3621 \\
1.3242 \\
1.3718 \\
1.4226 \\
1.4310 \\
1.4118\end{array}$ \\
\hline
\end{tabular}

A-2 


\begin{tabular}{|c|c|c|c|c|c|c|c|}
\hline SAMPLE & $x$ & $Y$ & $\mathbf{z}$ & WCS & WCR & ALPHA & BETA \\
\hline 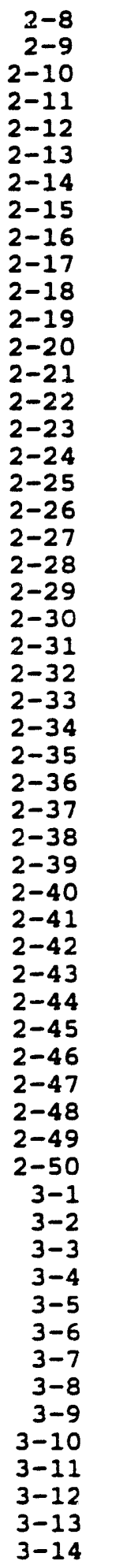 & $\begin{array}{r}-0.28 \\
0.22 \\
0.72 \\
1.22 \\
1.72 \\
2.22 \\
2.72 \\
3.22 \\
3.72 \\
4.22 \\
4.72 \\
5.22 \\
5.72 \\
6.22 \\
6.72 \\
7.22 \\
7.72 \\
8.22 \\
8.72 \\
3.22 \\
9.72 \\
10.22 \\
10.72 \\
11.22 \\
11.72 \\
12.22 \\
12.72 \\
13.22 \\
13.72 \\
14.22 \\
14.72 \\
15.22 \\
1.72 \\
15.72 \\
1.72 \\
16.22 \\
16.72 \\
17.22 \\
-0.72 \\
17.72 \\
18.22 \\
18.72 \\
19.22 \\
19.72 \\
20.22 \\
20.72 \\
-3.78 \\
-3.28 \\
-2.78 \\
-2.28 \\
-1.78 \\
1.28 \\
132\end{array}$ & $\begin{array}{l}-0.60 \\
-0.60 \\
-0.60 \\
-0.60 \\
-0.60 \\
-0.60 \\
-0.60 \\
-0.60 \\
-0.60 \\
-0.60 \\
-0.60 \\
-0.60 \\
-0.60 \\
-0.60 \\
-0.60 \\
-0.60 \\
-0.60 \\
-0.60 \\
-0.60 \\
-0.60 \\
-0.60 \\
-0.60 \\
-0.60 \\
-0.60 \\
-0.60 \\
-0.60 \\
-0.60 \\
-0.60 \\
-0.60 \\
-0.60 \\
-0.60 \\
-0.60 \\
-0.60 \\
-0.60 \\
-0.60 \\
-0.60 \\
-0.0 .60 \\
-0.60 \\
-0.60 \\
-0.60 \\
-0.60 \\
-0.60 \\
-0.60 \\
-0.60 \\
-0.60 \\
-0.60 \\
-0.60 \\
-0.60 \\
-0.60 \\
-0.60 \\
-0.60 \\
-0.60 \\
-0.60 \\
-0.60 \\
-0.60\end{array}$ & 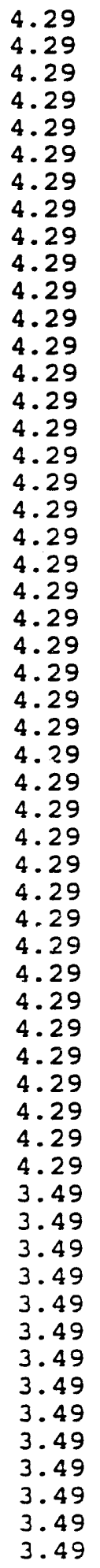 & $\begin{array}{l}0.297 \\
0.280 \\
0.288 \\
0.351 \\
0.338 \\
0.357 \\
0.356 \\
0.348 \\
0.385 \\
0.305 \\
0.369 \\
0.371 \\
0.314 \\
0.298 \\
0.309 \\
0.360 \\
0.325 \\
0.353 \\
0.339 \\
0.375 \\
0.351 \\
0.324 \\
0.303 \\
0.337 \\
-999 \\
0.399 \\
0.370 \\
0.388 \\
0.366 \\
0.370 \\
0.375 \\
0.394 \\
0.388 \\
0.353 \\
0.375 \\
0.380 \\
0.338 \\
0.371 \\
0.370 \\
0.999 \\
0.330 \\
0.376 \\
0.312 \\
0.326 \\
0.372 \\
0.395 \\
0.369 \\
0.355 \\
0.360 \\
0.320 \\
0.337 \\
0.350 \\
0.354 \\
0.396\end{array}$ & $\begin{array}{l}0.0350 \\
0.0350 \\
0.0350 \\
0.0350 \\
0.0350 \\
0.0350 \\
0.0350 \\
0.0350 \\
0.0350 \\
0.0350 \\
0.0350 \\
0.0000 \\
0.0350 \\
0.0350 \\
0.0350 \\
0.0350 \\
0.0350 \\
0.0350 \\
0.0350 \\
0.0350 \\
0.0350 \\
0.0350 \\
0.0350 \\
0.0350 \\
-999 \\
0.0350 \\
0.0350 \\
0.0350 \\
0.0350 \\
0.0350 \\
0.0350 \\
0.0350 \\
0.0350 \\
0.0350 \\
0.0350 \\
0.0350 \\
0.0350 \\
0.0350 \\
0.0350 \\
-999 \\
0.0350 \\
0.0350 \\
0.0350 \\
0.0350 \\
0.0000 \\
0.0000 \\
0.0000 \\
0.0179 \\
0.0350 \\
0.0350 \\
0.0350 \\
0.0350 \\
0.0350\end{array}$ & 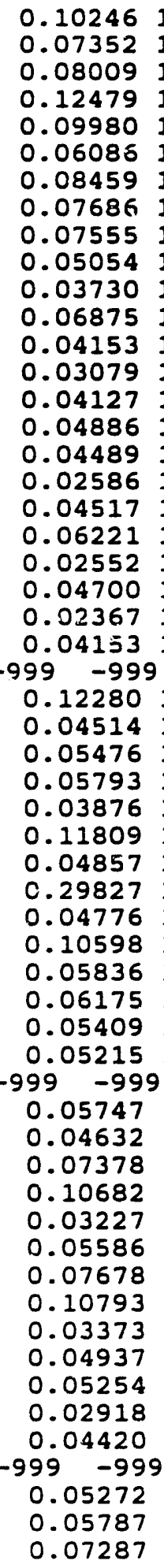 & $\begin{array}{r}1.3407 \\
1.4248 \\
1.3238 \\
1.3488 \\
1.3552 \\
1.3438 \\
1.3269 \\
1.3032 \\
1.3717 \\
1.3811 \\
1.2742 \\
1.1997 \\
1.3094 \\
1.4943 \\
1.4286 \\
1.4245 \\
1.3546 \\
1.3781 \\
1.2872 \\
1.3241 \\
1.3845 \\
1.5254 \\
1.4925 \\
1.4783 \\
9 \\
1.3761 \\
1.4876 \\
1.3473 \\
1.3784 \\
1.4126 \\
1.3409 \\
1.3572 \\
1.1655 \\
1.5674 \\
1.3015 \\
1.3218 \\
1.5231 \\
1.4769 \\
1.5047 \\
9 \\
1.4993 \\
1.3903 \\
1.5037 \\
1.18304 \\
1.2904 \\
1.2379 \\
1.2577 \\
1.2713 \\
1.5167 \\
1.3894 \\
1.3826 \\
1.4399 \\
1.4084 \\
-3999\end{array}$ \\
\hline
\end{tabular}




\begin{tabular}{|c|c|c|c|c|c|c|c|}
\hline SAMPLE & $\mathbf{x}$ & $\mathbf{Y}$ & $\mathbf{z}$ & WCS & WCR & ALPHA & BETA \\
\hline $\begin{array}{l}3-15 \\
3-16 \\
3-17 \\
3-18 \\
3-19 \\
3-20 \\
3-21 \\
3-22 \\
3-23 \\
3-24 \\
3-25 \\
3-26 \\
3-27 \\
3-28 \\
3-29 \\
3-30 \\
3-31 \\
3-32 \\
3-33 \\
3-34 \\
3-35 \\
3-36 \\
3-37 \\
3-38 \\
3-39 \\
3-40 \\
3-41 \\
3-42 \\
3-43 \\
3-44 \\
3-45 \\
3-46 \\
3-47 \\
3-48 \\
3-49 \\
3-50 \\
4-1 \\
4-16 \\
4-18 \\
4-19 \\
4-20\end{array}$ & $\begin{array}{r}3.22 \\
3.72 \\
4.22 \\
4.72 \\
5.22 \\
5.72 \\
6.22 \\
6.72 \\
7.22 \\
7.72 \\
8.22 \\
8.72 \\
9.22 \\
9.72 \\
10.22 \\
10.72 \\
11.22 \\
11.72 \\
12.22 \\
12.72 \\
13.22 \\
13.72 \\
14.22 \\
14.72 \\
15.22 \\
15.72 \\
16.22 \\
16.72 \\
17.22 \\
17.72 \\
18.22 \\
18.72 \\
19.22 \\
19.72 \\
19.72 \\
20.72 \\
5.72 \\
20.72 \\
10.72 \\
-3.72 \\
-3.28 \\
-2.72 \\
-2.28 \\
-1.78 \\
-1.28 \\
-0.78 \\
-0.28 \\
0.72\end{array}$ & $\begin{array}{l}-0.60 \\
-0.60 \\
-0.60 \\
-0.60 \\
-0.60 \\
-0.60 \\
-0.60 \\
-0.60 \\
-0.60 \\
-0.60 \\
-0.60 \\
-0.60 \\
-0.60 \\
-0.60 \\
-0.60 \\
-0.60 \\
-0.60 \\
-0.60 \\
-0.60 \\
-0.60 \\
-0.60 \\
-0.60 \\
-0.60 \\
-0.60 \\
-0.60 \\
-0.60 \\
-0.60 \\
-0.60 \\
-0.60 \\
-0.60 \\
-0.60 \\
-0.60 \\
-0.60 \\
-0.60 \\
-0.60 \\
-0.60 \\
-0.60 \\
-0.60 \\
-0.60 \\
-0.60 \\
-0.60 \\
-0.0 .60 \\
-0.60 \\
-0.60 \\
-0.60 \\
-0.60 \\
-0.60 \\
-0.60 \\
-0.60 \\
-0.60\end{array}$ & 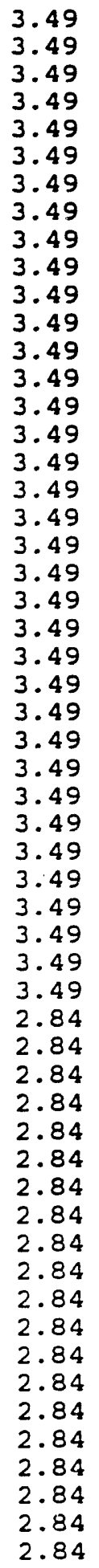 & $\begin{array}{l}0.321 \\
0.318 \\
0.369 \\
0.262 \\
0.350 \\
0.328 \\
0.309 \\
-999 \\
0.332 \\
0.305 \\
0.353 \\
0.305 \\
0.302 \\
0.314 \\
0.302 \\
0.323 \\
0.310 \\
0.333 \\
0.319 \\
0.337 \\
0.357 \\
0.312 \\
0.344 \\
0.312 \\
0.330 \\
0.334 \\
0.306 \\
0.347 \\
0.361 \\
0.361 \\
0.329 \\
0.352 \\
0.321 \\
0.361 \\
0.347 \\
0.316 \\
0.293 \\
0.305 \\
0.283 \\
0.287 \\
0.297 \\
0.307 \\
0.309 \\
0.291 \\
0.267 \\
0.343 \\
0.306 \\
0.323 \\
0.303 \\
0.301 \\
0.3781 \\
0.294\end{array}$ & $\begin{array}{l}0.0350 \\
0.0350 \\
0.0350 \\
0.0350 \\
0.0350 \\
0.0350 \\
0.0350 \\
-999 \\
0.0350 \\
0.0350 \\
0.0350 \\
0.0350 \\
0.0350 \\
0.0350 \\
0.0350 \\
0.0350 \\
0.0350 \\
0.0350 \\
0.0350 \\
0.0350 \\
0.0350 \\
0.0350 \\
0.0350 \\
0.0350 \\
0.0350 \\
0.0350 \\
0.0350 \\
0.0350 \\
0.0350 \\
0.0350 \\
0.0350 \\
0.0350 \\
0.0350 \\
0.0350 \\
0.0350 \\
0.0350 \\
0.0000 \\
0.0000 \\
0.0250 \\
0.0250 \\
0.0250 \\
0.0250 \\
0.0000 \\
0.0250 \\
0.0000 \\
0.0250 \\
0.0250 \\
0.0250 \\
0.0250 \\
0.0250 \\
0.0250 \\
-999 \\
-999 \\
0.0250 \\
0.0250 \\
0.0250\end{array}$ & 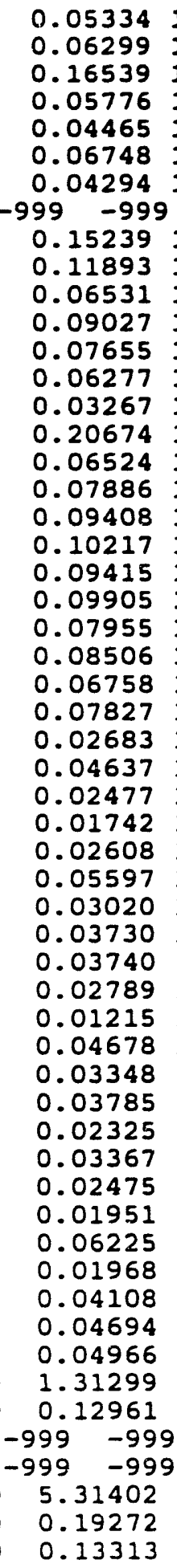 & $\begin{array}{r}1.3029 \\
1.3500 \\
1.2658 \\
1.4145 \\
1.4883 \\
1.4372 \\
1.5486 \\
-999 \\
1.4936 \\
1.4160 \\
1.4480 \\
1.4064 \\
1.3774 \\
1.5159 \\
1.7437 \\
1.3469 \\
1.4893 \\
1.5705 \\
1.4733 \\
1.5730 \\
1.6320 \\
1.5552 \\
1.7063 \\
1.6141 \\
1.6289 \\
1.6134 \\
1.6158 \\
1.4010 \\
1.3775 \\
1.5212 \\
1.5253 \\
1.5630 \\
1.3126 \\
1.3798 \\
1.4206 \\
1.3564 \\
1.3198 \\
1.4692 \\
1.1 .3250 \\
1.6832 \\
1.5882 \\
1.5715 \\
1.5439 \\
1.4011 \\
1.4064 \\
1.1364 \\
1.3904 \\
1.3612 \\
1.3935 \\
1.3666\end{array}$ \\
\hline
\end{tabular}




\begin{tabular}{|c|c|c|c|c|c|c|c|}
\hline SAMPLE & $x$ & $\mathbf{Y}$ & $\mathbf{z}$ & WCS & WCR & ALPHA & BETA \\
\hline $\begin{array}{r}4-21 \\
4-22 \\
4-23 \\
4-24 \\
4-25 \\
4-26 \\
4-27 \\
4-28 \\
4-29 \\
4-30 \\
4-31 \\
4-32 \\
4-33 \\
4-34 \\
4-35 \\
4-36 \\
4-37 \\
4-38 \\
4-39 \\
4-40 \\
4-41 \\
4-42 \\
4-{ }\end{array}$ & $\begin{array}{r}6.22 \\
6.72 \\
7.22 \\
7.72 \\
8.22 \\
8.72 \\
9.22 \\
9.72 \\
10.22 \\
10.72 \\
11.22 \\
11.72 \\
12.22 \\
12.72 \\
13.22 \\
13.72 \\
14.22 \\
14.72 \\
15.22 \\
15.72 \\
16.22 \\
16.72 \\
17.22 \\
17.72 \\
18.22 \\
18.72 \\
19.22 \\
19.72 \\
20.22 \\
20.72 \\
-3.78 \\
-3.28 \\
-2.78 \\
-2.28 \\
-1.78 \\
-1.28 \\
-0.78 \\
-0.28 \\
0.22 \\
0.72 \\
1.22 \\
7.72 \\
1.72 \\
2.72 \\
9.22 \\
2.72 \\
3.22 \\
3.72 \\
4.22 \\
4.72 \\
5.22 \\
5.72 \\
6.22 \\
6.72 \\
9\end{array}$ & $\begin{array}{l}-0.60 \\
-0.60 \\
-0.60 \\
-0.60 \\
-0.60 \\
-0.60 \\
-0.60 \\
-0.60 \\
-0.60 \\
-0.60 \\
-0.60 \\
-0.60 \\
-0.60 \\
-0.60 \\
-0.60 \\
-0.60 \\
-0.60 \\
-0.60 \\
-0.60 \\
-0.60 \\
-0.60 \\
-0.60 \\
-0.60 \\
-0.60 \\
-0.60 \\
-0.60 \\
-0.60 \\
-0.60 \\
-0.60 \\
-0.60 \\
-0.60 \\
-0.60 \\
-0.60 \\
-0.0 .60 \\
-0.60 \\
-0.60 \\
-0.60 \\
-0.60 \\
-0.60 \\
-0.60 \\
-0.60 \\
-0.60 \\
-0.60 \\
-0.60 \\
-0.60 \\
-0.60 \\
-0.60 \\
-0.60 \\
-0.60 \\
-0.60 \\
-0.60 \\
-0.60 \\
-0.60 \\
-0.60\end{array}$ & 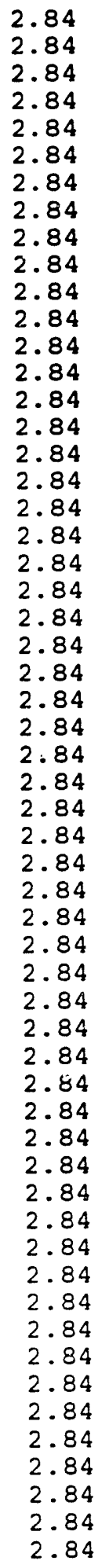 & $\begin{array}{l}0.290 \\
0.361 \\
0.263 \\
0.349 \\
0.315 \\
0.292 \\
0.294 \\
0.307 \\
-999 \\
0.348 \\
0.312 \\
0.326 \\
0.342 \\
0.346 \\
0.305 \\
0.294 \\
0.308 \\
0.334 \\
0.317 \\
0.349 \\
0.276 \\
0.296 \\
0.291 \\
0.325 \\
0.371 \\
0.307 \\
0.303 \\
0.281 \\
0.281 \\
0.316 \\
0.299 \\
0.288 \\
0.331 \\
0.310 \\
0.306 \\
0.312 \\
0.269 \\
0.318 \\
0.328 \\
0.314 \\
0.272 \\
0.286 \\
0.327 \\
0.252 \\
0.297 \\
0.312 \\
0.355 \\
0.327 \\
0.318 \\
0.319 \\
0.282 \\
0.319 \\
0.305 \\
0.306 \\
0.290 \\
0.287 \\
0.278\end{array}$ & 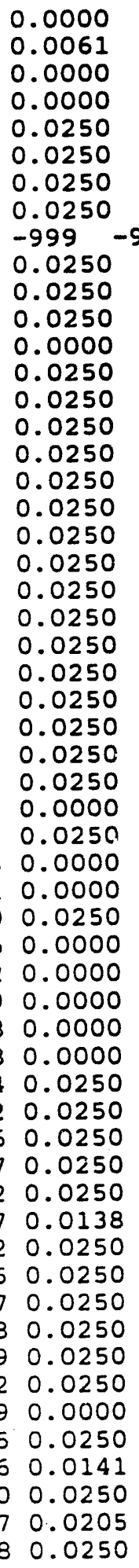 & $\begin{array}{l}0.03778 \\
0.05257 \\
0.04187 \\
0.07569 \\
0.04542 \\
0.02927 \\
0.03867 \\
0.07259 \\
999 \\
0.07549 \\
0.04938 \\
0.12628 \\
0.03840 \\
0.06393 \\
0.07566 \\
0.04428 \\
0.10927 \\
0.67592 \\
0.15022 \\
0.84037 \\
0.06243 \\
0.08345 \\
0.02760 \\
0.05697 \\
0.02357 \\
0.02775 \\
0.03912 \\
0.02082 \\
0.03113 \\
0.05763 \\
0.05288 \\
0.04653 \\
0.02953 \\
0.03715 \\
0.08504 \\
0.04767 \\
0.02704 \\
0.01091 \\
0.04053 \\
0.04113 \\
0.04004 \\
0.02684 \\
0.07158 \\
0.03436 \\
0.19335 \\
0.06933 \\
0.09316 \\
0.06955 \\
0.05323 \\
0.07631 \\
0.02632 \\
0.017129 \\
0.06349 \\
0.0405436 \\
0.02535\end{array}$ & 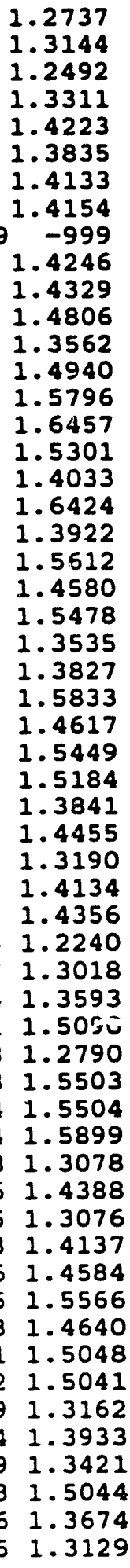 \\
\hline
\end{tabular}

A-5 


\begin{tabular}{|c|c|c|c|c|c|c|c|}
\hline SAMPLE & $\mathbf{x}$ & $Y$ & $z$ & wCS & WCR & ALPHA & BETA \\
\hline 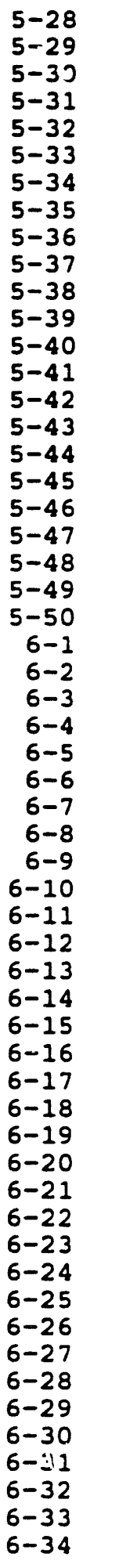 & $\begin{array}{r}9.72 \\
10.22 \\
10.72 \\
11.22 \\
11.72 \\
12.22 \\
12.72 \\
13.22 \\
13.72 \\
14.22 \\
14.72 \\
15.22 \\
15.72 \\
16.22 \\
16.72 \\
17.22 \\
17.72 \\
18.22 \\
18.72 \\
19.22 \\
19.72 \\
20.22 \\
20.72 \\
-3.78 \\
-3.28 \\
-2.78 \\
-2.28 \\
-1.78 \\
-1.28 \\
-0.78 \\
-0.28 \\
0.22 \\
10.22 \\
10.72 \\
11.22 \\
11.72 \\
12.22 \\
12.72 \\
1.22 \\
1.72 \\
2.22 \\
2.72 \\
3.72 \\
3.22 \\
3.72 \\
4.22 \\
4.72 \\
5.22 \\
5.72 \\
6.72 \\
7.22 \\
12.72\end{array}$ & $\begin{array}{l}-0.60 \\
-0.60 \\
-0.60 \\
-0.60 \\
-0.60 \\
-0.60 \\
-0.60 \\
-0.60 \\
-0.60 \\
-0.60 \\
-0.60 \\
-0.60 \\
-0.60 \\
-0.60 \\
-0.60 \\
-0.60 \\
-0.60 \\
-0.60 \\
-0.60 \\
-0.60 \\
-0.60 \\
-0.60 \\
-0.60 \\
-0.60 \\
-0.60 \\
-0.60 \\
-0.60 \\
-0.60 \\
-0.60 \\
-0.60 \\
-0.60 \\
-0.60 \\
-0.60 \\
-0.60 \\
-0.60 \\
-0.60 \\
-0.60 \\
-0.60 \\
-0.60 \\
-0.60 \\
-0.60 \\
-0.60 \\
-0.60 \\
-0.60 \\
-0.60 \\
-0.60 \\
-0.60 \\
-0.60 \\
-0.60 \\
-0.60\end{array}$ & $\begin{array}{l}2.84 \\
2.84 \\
2.84 \\
2.84 \\
2.84 \\
2.84 \\
2.84 \\
2.84 \\
2.84 \\
2.84 \\
2.84 \\
2.84 \\
2.84 \\
2.84 \\
2.84 \\
2.84 \\
2.84 \\
2.84 \\
2.84 \\
2.84 \\
2.84 \\
2.84 \\
2.84 \\
1.79 \\
1.79 \\
1.79 \\
1.79 \\
1.79 \\
1.79 \\
1.79 \\
1.79 \\
1.79 \\
1.79 \\
1.79 \\
1.79 \\
1.79 \\
1.79 \\
1.79 \\
1.79 \\
1.79 \\
1.79 \\
1.79 \\
1.79 \\
1.79 \\
1.79 \\
1.79 \\
1.79 \\
1.79 \\
1.79 \\
1.79 \\
1.79 \\
1.79 \\
1.79\end{array}$ & $\begin{array}{l}0.309 \\
0.274 \\
-999 \\
0.297 \\
0.297 \\
0.276 \\
0.305 \\
0.362 \\
0.304 \\
0.302 \\
0.311 \\
0.327 \\
-999 \\
0.328 \\
0.308 \\
0.284 \\
0.287 \\
0.296 \\
0.320 \\
0.338 \\
0.325 \\
0.320 \\
0.338 \\
0.324 \\
0.353 \\
0.345 \\
0.277 \\
0.434 \\
-999 \\
0.280 \\
0.290 \\
0.314 \\
0.235 \\
0.315 \\
0.323 \\
0.306 \\
0.312 \\
0.283 \\
0.286 \\
0.293 \\
0.283 \\
0.287 \\
0.288 \\
0.240 \\
0.305 \\
0.296 \\
0.314 \\
0.335 \\
0.315 \\
0.305 \\
0.303 \\
0.310 \\
0.286 \\
0.278 \\
0.297 \\
0.301 \\
0.282\end{array}$ & $\begin{array}{l}0.0250 \\
0.0000 \\
-999 \\
0.0250 \\
0.0250 \\
0.0000 \\
0.0250 \\
0.0250 \\
0.0250 \\
0.0250 \\
0.0250 \\
0.0000 \\
-999 \\
0.0250 \\
0.0250 \\
0.0250 \\
0.0250 \\
0.0250 \\
0.0250 \\
0.0000 \\
0.0250 \\
0.0214 \\
0.0250 \\
0.0350 \\
0.0000 \\
0.0350 \\
0.0000 \\
0.0000 \\
-999 \\
0.0350 \\
0.0350 \\
0.0350 \\
0.0350 \\
0.0350 \\
0.0350 \\
0.0350 \\
0.0350 \\
0.0350 \\
0.0350 \\
0.0350 \\
0.0350 \\
0.0350 \\
0.0350 \\
0.0350 \\
0.0350 \\
0.035030 \\
0.030350 \\
0.0350 \\
0.0350 \\
0.0350 \\
0.0350 \\
0.030 \\
0.030 \\
0.030 \\
0.030 \\
0.030 \\
0.030\end{array}$ & 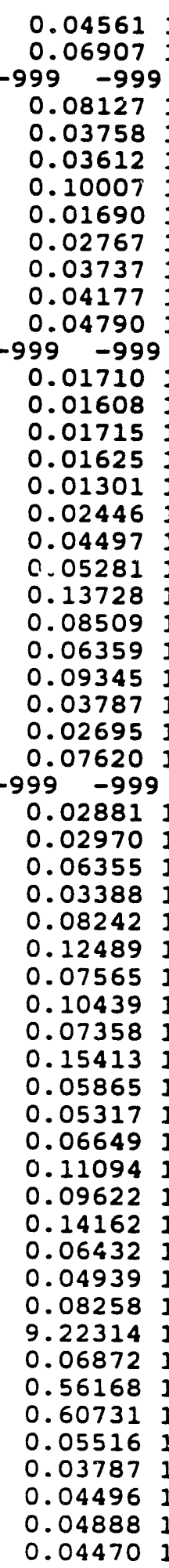 & $\begin{array}{l}1.3035 \\
1.1966 \\
-999 \\
1.3784 \\
1.4749 \\
1.3418 \\
1.2688 \\
1.3170 \\
1.4413 \\
1.4573 \\
1.4525 \\
1.3127 \\
-9999 \\
1.4991 \\
1.5428 \\
1.4959 \\
1.5344 \\
1.6336 \\
1.4967 \\
1.2965 \\
1.4034 \\
1.4008 \\
1.3334 \\
1.1956 \\
1.1421 \\
1.2330 \\
1.3199 \\
1.1029 \\
1.4999 \\
1.4280 \\
1.4817 \\
1.2882 \\
1.5130 \\
1.2772 \\
1.2127 \\
1.2778 \\
1.5025 \\
1.5873 \\
1.4667 \\
1.5615 \\
1.43444 \\
1.5564 \\
1.7106 \\
1.7199 \\
1.5667 \\
1.5613 \\
1.9278 \\
1.5643 \\
1.2420 \\
1.5492 \\
1.5534 \\
1.7995 \\
1.7304 \\
1.6971\end{array}$ \\
\hline
\end{tabular}




\begin{tabular}{|c|c|c|c|c|c|c|c|}
\hline SAMPLE & $x$ & $Y$ & 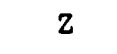 & WCS & WCR & ALPHA & BETA \\
\hline 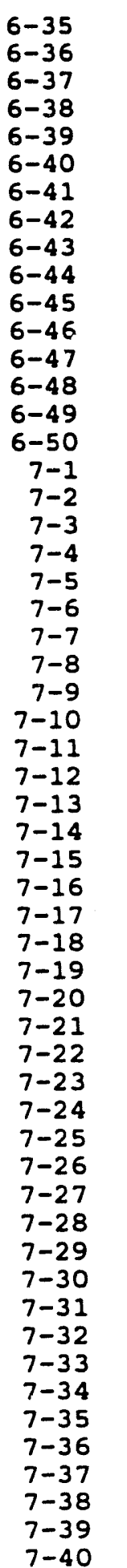 & $\begin{array}{r}13.22 \\
13.72 \\
14.22 \\
14.72 \\
15.22 \\
15.72 \\
16.22 \\
16.72 \\
17.22 \\
17.72 \\
18.22 \\
18.72 \\
19.22 \\
19.72 \\
20.22 \\
20.72 \\
-3.78 \\
-3.28 \\
-2.78 \\
-2.28 \\
-1.78 \\
-1.28 \\
-0.78 \\
-0.28 \\
0.22 \\
0.72 \\
1.22 \\
1.72 \\
2.22 \\
2.72 \\
3.22 \\
3.72 \\
4.22 \\
4.72 \\
5.22 \\
5.72 \\
13.72 \\
14.22 \\
14.72 \\
15.22 \\
15.72\end{array}$ & $\begin{array}{l}-0.60 \\
-0.60 \\
-0.60 \\
-0.60 \\
-0.60 \\
-0.60 \\
-0.60 \\
-0.60 \\
-0.60 \\
-0.60 \\
-0.60 \\
-0.60 \\
-0.60 \\
-0.60 \\
-0.60 \\
-0.60 \\
-0.60 \\
-0.60 \\
-0.60 \\
-0.60 \\
-0.60 \\
-0.60 \\
-0.60 \\
-0.60 \\
-0.60 \\
-0.60 \\
-0.60 \\
-0.60 \\
-0.60 \\
-0.60 \\
-0.60 \\
-0.60 \\
-0.60 \\
-0.60 \\
-0.60 \\
-0.60 \\
-0.60 \\
-0.60 \\
-0.60 \\
-0.60 \\
-0.60 \\
-0.60 \\
-0.60 \\
-0.60 \\
-0.60 \\
-0.60 \\
-0.60 \\
-0.60 \\
-0.60\end{array}$ & $\begin{array}{l}1.79 \\
1.79 \\
1.79 \\
1.79 \\
1.79 \\
1.79 \\
1.79 \\
1.79 \\
1.79 \\
1.79 \\
1.79 \\
1.79 \\
11 \\
1.79 \\
1.79 \\
1.79 \\
1.79 \\
1.02 \\
1.02 \\
1.02 \\
1.02 \\
1.02 \\
1.02 \\
1.02\end{array}$ & $\begin{array}{l}0.297 \\
0.245 \\
0.274 \\
0.281 \\
0.291 \\
0.287 \\
0.300 \\
0.218 \\
0.287 \\
0.260 \\
0.289 \\
0.287 \\
0.280 \\
0.307 \\
0.273 \\
0.329 \\
0.340 \\
0.320 \\
0.343 \\
0.339 \\
0.308 \\
0.323 \\
0.331 \\
0.350 \\
0.356 \\
0.333 \\
0.299 \\
0.332 \\
0.311 \\
0.327 \\
0.310 \\
0.296 \\
0.307 \\
0.316 \\
0.331 \\
0.351 \\
0.322 \\
0.326 \\
0.301 \\
0.322 \\
0.309 \\
0.323 \\
0.393 \\
0.355 \\
0.296 \\
0.308 \\
0.329 \\
0.399 \\
0.39 \\
0.300 \\
0.319\end{array}$ & $\begin{array}{l}0.0000 \\
0.0267 \\
0.0350 \\
0.0350 \\
0.0341 \\
0.0350 \\
0.0350 \\
0.0350 \\
0.0350 \\
0.0300 \\
0.0350 \\
0.0000 \\
0.0350 \\
0.0350 \\
0.0350 \\
0.0319 \\
0.0000 \\
0.0000 \\
0.0000 \\
0.0000 \\
0.0000 \\
0.0450 \\
0.0000 \\
0.0000 \\
0.0000 \\
0.0000 \\
0.0450 \\
0.000 \\
0.0000 \\
0.0450 \\
0.00000 \\
0.0450 \\
0.0000 \\
0.0000 \\
0.0000 \\
0.0000 \\
0.0000 \\
0.0000 \\
0.0000 \\
0.0000 \\
0.0000 \\
0.0450 \\
0.0450 \\
0.0000 \\
0.0450 \\
0.0000 \\
0.0000 \\
0.000 \\
0.000 \\
0.000 \\
0.000 \\
0.000 \\
0.000 \\
0.000 \\
0.000 \\
0.000\end{array}$ & $\begin{array}{c}0.05068 \\
0.04041 \\
0.11881 \\
0.06384 \\
0.04342 \\
0.04579 \\
0.03631 \\
0.02562 \\
0.06589 \\
0.09048 \\
0.05824 \\
0.05224 \\
0.03663 \\
0.06903 \\
0.07438 \\
0.08314 \\
0.15335 \\
0.03772 \\
0.05522 \\
0.06051 \\
0.01452 \\
0.01133 \\
0.05406 \\
0.27426 \\
0.04934 \\
0.16264 \\
0.03203 \\
0.09616 \\
0.00768 \\
0.03755 \\
0.02909 \\
0.01348 \\
0.03405 \\
0.14227 \\
0.14257 \\
0.15798 \\
0.12351 \\
0.02959 \\
0.04823 \\
0.03859 \\
0.01759 \\
0.01992 \\
0.06239 \\
0.03237 \\
0.05088 \\
0.05488 \\
0.04081 \\
0.04956936 \\
0.05906 \\
0.02026 \\
0.02651\end{array}$ & 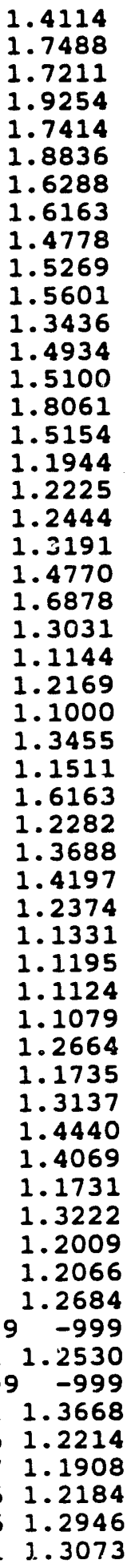 \\
\hline
\end{tabular}




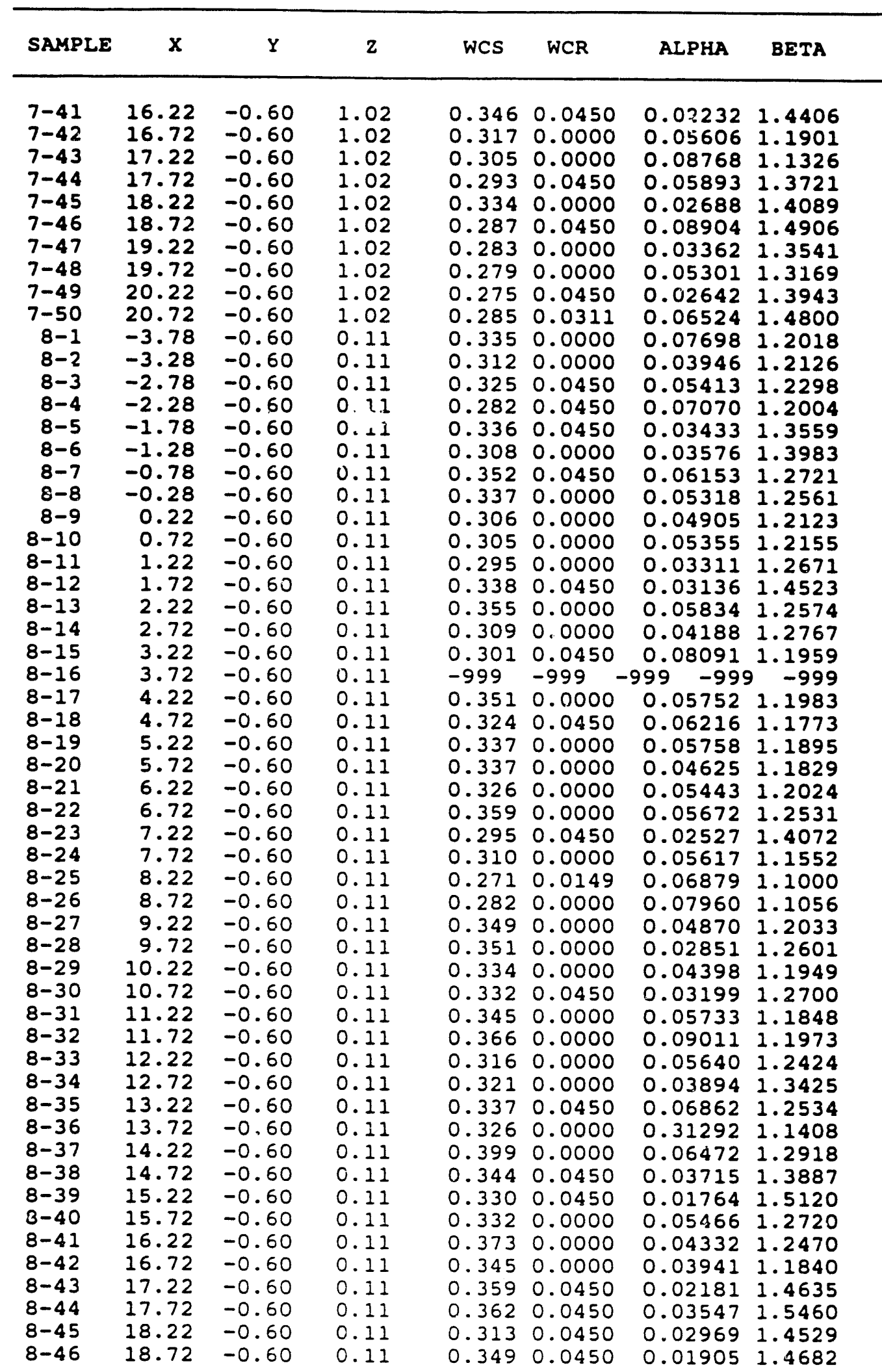




\begin{tabular}{|c|c|c|c|c|c|c|c|}
\hline SAMPLE & $\mathbf{x}$ & $Y$ & 2 & WCS & WCR & ALPHA & BETA \\
\hline $\begin{array}{r}8-47 \\
8-48 \\
8-49 \\
8-50 \\
9-1 \\
9-2 \\
9-3 \\
9-4 \\
9-4 \\
9-5 \\
9-6 \\
9-7 \\
9-8 \\
9- \\
9-9 \\
9-10 \\
9-11 \\
9-12 \\
9- \\
9-13 \\
9-14 \\
9-15 \\
9-\end{array}$ & $\begin{array}{l}19.22 \\
19.72 \\
20.22 \\
20.72 \\
-3.78 \\
-3.28 \\
-2.78 \\
-2.28 \\
-1.78 \\
-1.28 \\
-0.78 \\
-0.28 \\
0.22 \\
0.72 \\
1.22 \\
1.72 \\
2.22 \\
2.72 \\
3.22 \\
3.72 \\
4.22 \\
4.72 \\
5.22 \\
5.72 \\
6.22 \\
6.72 \\
7.22 \\
7.72 \\
8.22 \\
8.72 \\
9.22 \\
9.72 \\
10.22 \\
10.72 \\
11.22 \\
11.72 \\
12.22 \\
12.72 \\
13.22 \\
13.72 \\
14.22 \\
14.72 \\
15.22 \\
15.72 \\
16.22 \\
16.72 \\
17.22 \\
17.72 \\
18.22 \\
18.72 \\
20.72\end{array}$ & $\begin{array}{l}-0.60 \\
-0.60 \\
-0.60 \\
-0.60 \\
-0.60 \\
-0.60 \\
-0.60 \\
-0.60 \\
-0.60 \\
-0.60 \\
-0.60 \\
-0.60 \\
-0.60 \\
-0.60 \\
-0.60 \\
-0.60 \\
-0.60 \\
-0.60 \\
-0.60 \\
-0.60 \\
-0.60 \\
-0.60 \\
-0.60 \\
-0.60 \\
-0.60 \\
-0.60 \\
-0.60 \\
-0.60 \\
-0.60 \\
-0.60 \\
-0.60 \\
-0.60 \\
-0.60 \\
-0.60 \\
-0.60 \\
-0.60 \\
-0.60 \\
-0.60 \\
-0.60 \\
-0.60 \\
-0.60 \\
-0.60 \\
-0.60 \\
-0.60 \\
-0.60 \\
-0.60 \\
-0.60 \\
-0.60\end{array}$ & $\begin{array}{l}0.11 \\
0.11 \\
0.11 \\
0.11 \\
-0.79 \\
-0.79 \\
-0.79 \\
-0.79 \\
-0.79 \\
-0.79 \\
-0.79 \\
-0.79 \\
-0.79 \\
-0.79 \\
-0.79 \\
-0.79 \\
-0.79 \\
-0.79 \\
-0.79 \\
-0.79 \\
-0.79 \\
-0.79 \\
-0.79 \\
-0.79 \\
-0.79 \\
-0.79 \\
-0.79 \\
-0.79 \\
-0.79 \\
-0.79 \\
-0.79 \\
-0.79 \\
-0.79 \\
-0.79 \\
-0.79 \\
-0.79 \\
-0.79 \\
-0.79 \\
-0.79 \\
-0.79 \\
-0.79 \\
-0.79 \\
-0.79 \\
-0.79 \\
-0.79 \\
-0.79 \\
-0.79 \\
-0.79 \\
-0.79 \\
-0.79 \\
-0.79 \\
-0.79 \\
-0.79 \\
-0.79\end{array}$ & 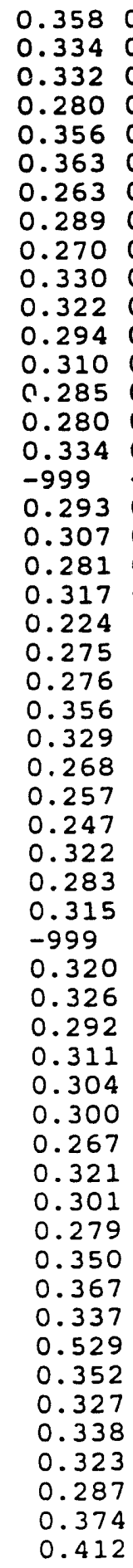 & 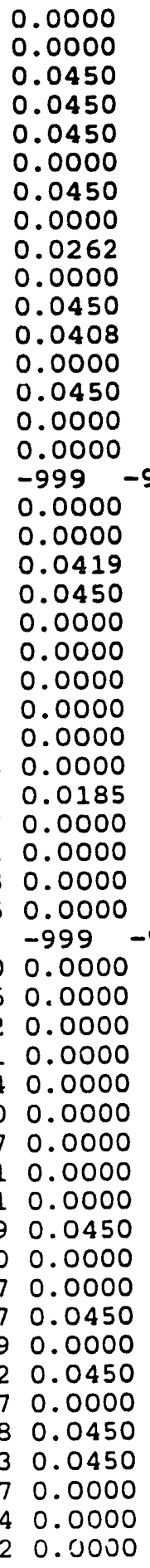 & $\begin{array}{c}0.11636 \\
0.15403 \\
0.10218 \\
0.05096 \\
0.01705 \\
0.01309 \\
0.01687 \\
0.04198 \\
0.04477 \\
0.02811 \\
0.03409 \\
0.05198 \\
0.03789 \\
0.01860 \\
0.03841 \\
0.06312 \\
099 \\
0.05591 \\
0.05472 \\
0.02404 \\
0.03590 \\
0.05302 \\
0.05688 \\
0.14881 \\
0.04943 \\
0.04888 \\
0.03917 \\
0.05336 \\
0.05149 \\
0.04907 \\
0.02446 \\
0.05882 \\
-999 \\
0.01929 \\
0.05168 \\
0.05462 \\
0.03728 \\
0.03433 \\
0.05688 \\
0.07091 \\
0.09065 \\
0.53732 \\
0.11277 \\
0.33724 \\
0.62708 \\
0.11368 \\
0.27160 \\
0.09061 \\
1.06125 \\
0.12384 \\
0.10534 \\
0.05966 \\
0.05615 \\
0.06479\end{array}$ & 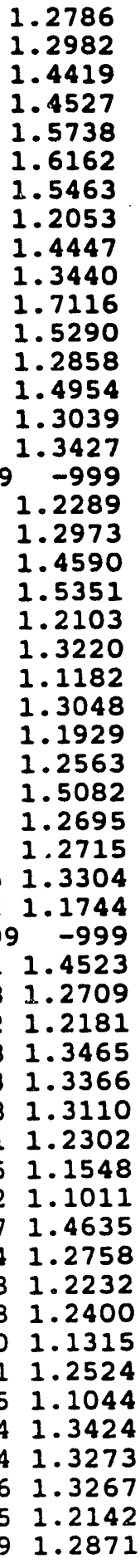 \\
\hline
\end{tabular}




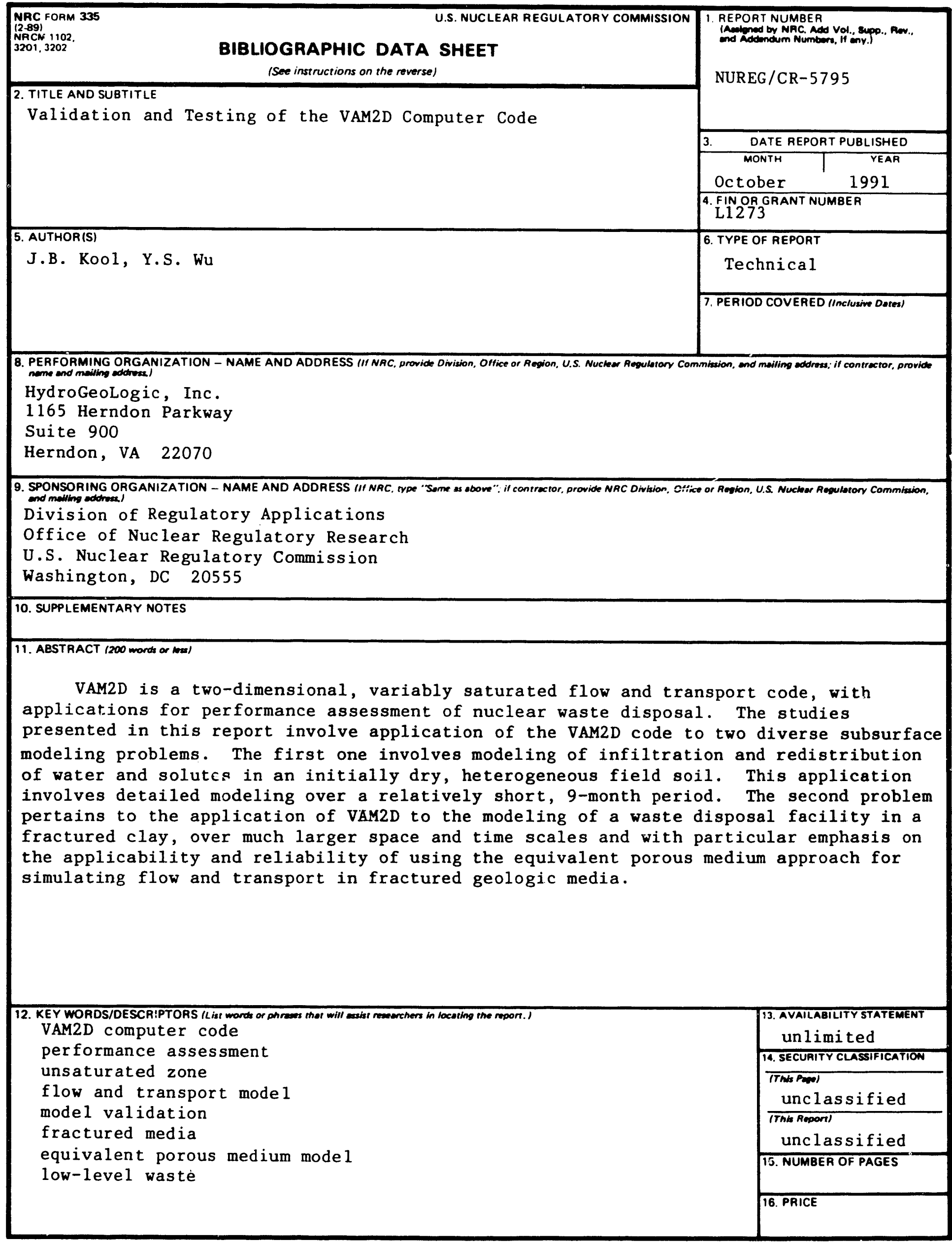



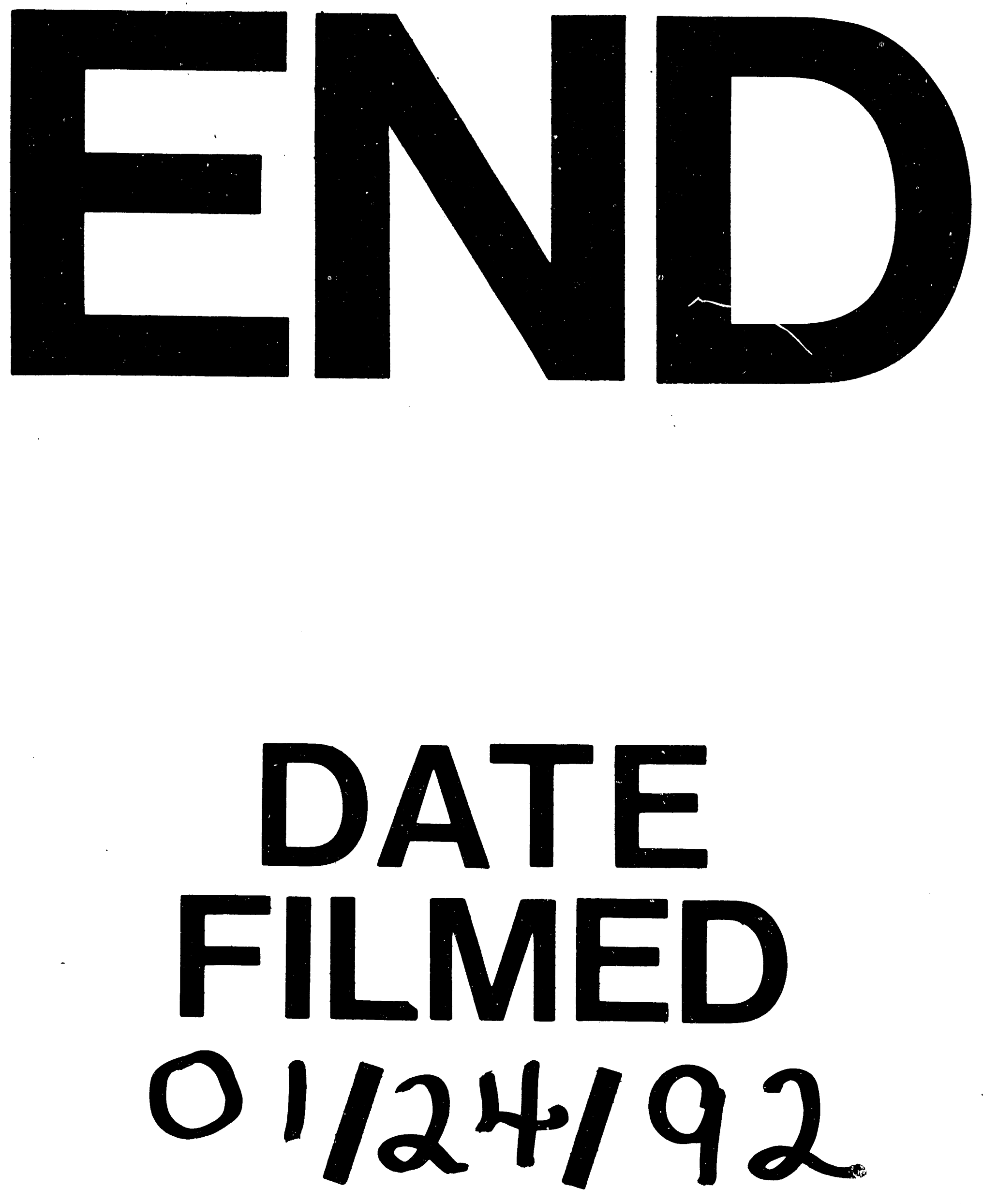

? 
. 